\title{
Fully differential NNLO computations with MATRIX
}

\author{
Massimiliano Grazzini ${ }^{1, \mathrm{a}}$, Stefan Kallweit ${ }^{2, \mathrm{~b}}$, Marius Wiesemann ${ }^{2, \mathrm{c}}$ \\ ${ }^{1}$ Physik-Institut, Universität Zürich, 8057 Zurich, Switzerland \\ 2 TH Division, Physics Department, CERN, 1211 Geneva 23, Switzerland
}

Received: 2 December 2017 / Accepted: 27 March 2018 / Published online: 29 June 2018

(C) The Author(s) 2018

\begin{abstract}
We present the computational framework MATRIX (http://matrix.hepforge.org/) which allows us to evaluate fully differential cross sections for a wide class of processes at hadron colliders in next-to-next-to-leading order (NNLO) QCD. The processes we consider are $2 \rightarrow 1$ and $2 \rightarrow 2$ hadronic reactions involving Higgs and vector bosons in the final state. All possible leptonic decay channels of the vector bosons are included for the first time in the calculations, by consistently accounting for all resonant and nonresonant diagrams, off-shell effects and spin correlations. We briefly introduce the theoretical framework MATRIX is based on, discuss its relevant features and provide a detailed description of how to use MATRIX to obtain NNLO accurate results for the various processes. We report reference predictions for inclusive and fiducial cross sections of all the physics processes considered here and discuss their corresponding uncertainties. MATRIX features an automatic extrapolation procedure that allows us, for the first time, to control the systematic uncertainties inherent to the applied NNLO subtraction procedure down to the few permille level (or better).
\end{abstract}

\section{Contents}

1 Introduction .................. 2

2 NNLO computations in the MATRIX framework . . . 3

2.1 The $q_{T}$-subtraction formalism $\ldots \ldots \ldots 3$

2.2 Implementation within the MATRIX framework 3

3 Available processes in MAtrix ... . . . . . . 4

4 How to use Matrix . . . . . . . . . . . . . 4

4.1 Compilation and setup of a process . . . . . . 4

4.2 Compilation with arguments . . . . . . 6

4.3 General structure of a process folder . . . . . 6

4.4 Running a process . . . . . . . . . . . . 8

\footnotetext{
a e-mail: grazzini@physik.uzh.ch

b e-mail: stefan.kallweit@cern.ch

c e-mail: marius.wiesemann@cern.ch
}

4.4.1 Running with interactive shell . . . . . 8

4.4.2 Running with arguments . . . . . . . . 9 9

4.5 Configuration file . . . . . . . . . . 10

5 Settings of a MATRIX run . . . . . . . . . . 11

5.1 Process-independent settings . . . . . . . 11

5.1.1 Settings in parameter. dat . . . . . 11

5.1.2 Settings in model. dat . . . . . . . 17

5.1.3 Settings in distribution. dat . . . 18

5.2 Process-specific settings . . . . . . . . 19

5.2.1 Higgs boson production . . . . . . . 21

5.2 .2 Vector-boson production ...... . . 21

5.2.3 Diphoton and vector-boson plus photon production ............ 22

5.2.4 Vector-boson pair production . . . . . . 23

6 Phenomenological results . . . . . . . . . 28

6.1 Settings . . . . . . . . . . . . 28

6.2 Cross-section predictions . . . . . . . . 28

7 Systematic uncertainties of $q_{T}$ subtraction . . . . . 34

8 Summary .................. 38

Appendix A Predefined cuts . . . . . . . . . . 40

Appendix B Modifications of the $\mathrm{C}++$ code (advanced

user) .................. . . 40

B.1 General C++ commands . . . . . . . . . . 40

B.1.1 Access to particle groups . . . . . . . 40

B.1.2 Access to user-defined parameters . . . . 44

B.2 Definition of a new particle group . . . . . . . 45

B.3 Implementation of a new dynamic scale . . . . . 45

B.4 Implementation of a new user-defined cut . . . . 46

B.5 Implementation of a new observable for distributions ............... . . 46

Appendix C Troubleshooting . . . . . . . . . . . 47

C.1 Compiling on lxplus . . . . . . . . . . . . 47

C.2 Using a window manager on lxplus . . . . . . . 47

C.3 Problems with libquadmath . . . . . . . 47

References ............... . 47 


\section{Introduction}

Precision computations for standard model (SM) processes are vital for the rich physics programme at the LHC. The increasing amount of collected data pushes the experimental uncertainties down to the percent level, thereby demanding accurate predictions for many relevant physics processes. This holds not only for SM measurements. Also new-physics searches rely on a precise modelling of the SM backgrounds. In particular, the sensitivity to small deviations from the SM predictions directly depends on the size of theoretical uncertainties. Besides single vector-boson and Higgs boson production processes, vector-boson pair production is particularly important in that respect since anomalous triple gauge couplings would be first uncovered in cross sections and distributions of the diboson processes.

Precise SM computations require, in particular, the inclusion of QCD radiative corrections at the next-to-leading order (NLO), and if possible at the next-to-next-to-leading order (NNLO). NNLO QCD predictions for the simplest hadronic reactions have been available for quite some time. The pioneering computation of the inclusive cross section for vector-boson production was carried out in the 1990s [2]. The corresponding computation for Higgs boson production was performed about ten years later [3-5]. They were followed by the calculation of the rapidity distribution of vector bosons [6]. Shortly after, fully differential calculations for Higgs and vector-boson production started to appear [7-12]. This further step was essential to obtain realistic predictions since fully differential computations allow us to apply selection cuts on the produced boson and on its decay products, and to directly address all the relevant kinematic distributions. The last decade has seen a revolution in the field of NNLO computations for hadron collider processes: the calculations for associated production of a Higgs boson with a vector boson [13-16], Higgs boson production in bottomquark annihilation [17-20], top-mass effects in Higgs boson production [21-25], $H H$ [26,27], $\gamma \gamma$ [28,29], $Z \gamma$ [30-32], $W \gamma$ [31], $Z Z$ [33-35], $W^{+} W^{-}[36,37]$ and $W^{ \pm} Z[38,39]$ production have been completed. NNLO results have been achieved also for further important processes like top-quark pair $[40,41]$ and single top $[42,43]$ production, dijet production [44], Higgs production through vector-boson fusion [45], $H+$ jet [46-48], $\gamma+$ jet [49], $Z+$ jet [50,51] and $W+$ jet [52]. Despite this tremendous progress, at present, publicly available NNLO programs typically carry out fully differential NNLO computations for a limited set of specific processes. Examples are FEWZ [53] and DYNNLO [12] for vectorboson production, FEHIPRO [7,54] and HNNLO [9,11] for Higgs boson production, and $2 \gamma$ NNLO [28] for diphoton production. A notable exception is MCFM [55], which in its current release features an NNLO implementation of single vector-boson and Higgs boson production, associated pro- duction of a Higgs boson with a vector-boson, and diphoton production.

In this paper, we present the computational framework MATRIX, ${ }^{1}$ which features a parton-level Monte Carlo generator capable of computing fiducial cross sections and distributions for Higgs boson, vector-boson and vector-boson pair production processes up to NNLO in QCD. For the first time, we consider all possible leptonic decay channels of the vector bosons, and we include spin correlations and off-shell effects by accounting for all resonant and nonresonant diagrams, thereby allowing the user to apply realistic fiducial cuts directly on the phase-space of the respective leptonic final state. MATRIX achieves NNLO accuracy by using a process-independent implementation of the $q_{T}$ subtraction formalism [9] in combination with a fully automated implementation of the Catani-Seymour dipole subtraction method [56,57] within the Monte Carlo program MUNICH. ${ }^{2}$ All (spin- and colour-correlated) tree-level and one-loop amplitudes are obtained from OPENLOOPS $[58,59]$. Early versions of MATRIX have been used, in combination with the two-loop scattering amplitudes of Refs. [6062], for the NNLO calculations of $Z \gamma[30,31], W^{ \pm} \gamma$ [31] $Z Z[33,34], W^{+} W^{-}[36,37], W^{ \pm} Z[38,39]$ and $H H$ [27] production $^{3}$ and the importance of including NNLO corrections for these processes is evident for both total rates and differential distributions. MATRIX provides a fully automated extrapolation procedure that allows us, for the first time, to control the systematic uncertainties inherent to the $q_{T}$-subtraction procedure down to the few permille level (or better) for all NNLO predictions of inclusive or fiducial cross sections. The MATRIX framework offers a simple interface to a powerful code to carry out such computations in a relatively straightforward way, and its first public version is now available for download [1].

The manuscript is organized as follows: In Sect. 2 we give a general introduction into the MATRIX framework, where we review the $q_{T}$-subtraction formalism and describe the organization of the computations. Section 3 provides the list of processes available in MATRIX. We then provide detailed instructions on how to use the code: This involves the generation, compilation and running of a process to compute LO, NLO and NNLO cross sections in Sect. 4, and a detailed description of the relevant input files and parameters in Sect. 5. In Sect. 6 we provide benchmark predictions for total and fiducial rates, respectively, for all processes, including the results of our

\footnotetext{
${ }^{1}$ MATRIX is the abbreviation of "MUNICH Automates qT subtraction and Resummation to Integrate X-sections".

2 MUNICH is the abbreviation of "MUlti-chaNnel Integrator at Swiss $(\mathrm{CH})$ precision" - an automated parton-level NLO generator by S. Kallweit.

${ }^{3}$ A first application of the code to the resummed transverse-momentum spectra of $Z Z$ and $W^{+} W^{-}$pairs has been presented in Ref. [63] at NNLL+NNLO.
} 
novel extrapolation procedure, and we discuss the relevant physics features of each process. A discussion of the systematic uncertainties of NNLO cross sections computed with $q_{T}$ subtraction for a representative set of processes and details on the extrapolation procedure are presented in Sect. 7. In Sect. 8 we summarize our results. All predefined phase-space cuts are listed in Appendix A. How to extend the predefined set of cuts, distributions and dynamic scales by modifying the underlying $\mathrm{C}++$ code is sketched in Appendix B. Finally, Appendix $\mathrm{C}$ provides a loose selection of solutions on compilation and running issues, which have been encountered in the testing phase of MATRIX and are expected to be potentially helpful for the user.

\section{NNLO computations in the MATRIX framework}

The computation of a QCD cross section at NNLO requires the evaluation of tree-level contributions with up to two additional unresolved partons, of one-loop contributions with one unresolved parton and of purely virtual contributions. The implementation of the corresponding scattering amplitudes in a complete NNLO calculation at the fully differential (exclusive) level is a highly non-trivial task because of the presence of infrared (IR) divergences at intermediate stages of the calculation. In particular, since the divergences affect real and virtual contributions in a different way, a straightforward combination of these components is not possible. Various methods have been proposed and used to overcome these issues at NNLO $[9,52,64-76]$. The method applied by MATRIX is transverse-momentum $\left(q_{T}\right)$ subtraction [9], and it is briefly described below.

\subsection{The $q_{T}$-subtraction formalism}

The $q_{T}$-subtraction formalism [9] is a method to handle and cancel IR divergences at NLO and NNLO. The method exploits the fact that for the production of a colourless finalstate system (i.e. a system composed of particles without QCD interactions) the behaviour of the $q_{T}$ distribution $^{4}$ at small $q_{T}$ has a universal (process-independent) structure that is explicitly known up to NNLO through the formalism of transverse-momentum resummation [77,78]. This knowledge is sufficient to fully determine the $q_{T}$ dependence of the cross section at small $q_{T}$ and to construct a non-local, but process-independent IR subtraction counterterm for this entire class of processes. ${ }^{5}$

\footnotetext{
${ }^{4}$ Here and in the following, $q_{T}$ always refers to the transverse momentum of the colourless final-state system under consideration.

5 The extension to heavy-quark production has been discussed in Ref. [79].
}

In the $q_{T}$-subtraction method, the cross section for a generic process $p p \rightarrow F+X$, where $F$ is a colourless system as specified above, can be written up to (N)NLO as

$\mathrm{d} \sigma_{(\mathrm{N}) \mathrm{NLO}}^{\mathrm{F}}=\mathcal{H}_{(\mathrm{N}) \mathrm{NLO}}^{\mathrm{F}} \otimes \mathrm{d} \sigma_{\mathrm{LO}}^{\mathrm{F}}+\left[\mathrm{d} \sigma_{(\mathrm{N}) \mathrm{LO}}^{\mathrm{F}+\mathrm{jet}}-\mathrm{d} \sigma_{(\mathrm{N}) \mathrm{NLO}}^{\mathrm{CT}}\right]$.

The term $d \sigma_{(\mathrm{N}) \mathrm{LO}}^{\mathrm{F} \text { jet }}$ represents the cross section for the production of the system $F+$ jet at $(\mathrm{N}) \mathrm{LO}$ accuracy. If Eq. (1) is applied at NLO, the $\mathrm{LO}$ cross section $d \sigma_{\mathrm{LO}}^{\mathrm{F}+\mathrm{jet}}$ can be obtained by direct integration of the corresponding tree-level amplitudes. If Eq. (1) is applied at NNLO, the NLO cross section $d \sigma_{\mathrm{NLO}}^{\mathrm{F}+\mathrm{jet}}$ can be evaluated by using any available NLO subtraction method [56,57,80,81] to handle and cancel the corresponding IR divergencies. Therefore, $d \sigma_{\mathrm{NLO}}^{\mathrm{F}+\mathrm{jet}}$ is finite provided that $q_{T} \neq 0$, but it diverges in the limit $q_{T} \rightarrow 0$. The process-independent counterterm $\mathrm{d} \sigma_{(\mathrm{N}) \mathrm{NLO}}^{\mathrm{CT}}$ guarantees the cancellation of this divergence of the $F+$ jet cross section, and its general expression is provided in Ref. [78]. The numerical implementation of the contribution in the square bracket in Eq. (1), which is by construction finite in the limit $q_{T} \rightarrow 0$, is discussed in detail in Sect. 2.2. The computation is completed by evaluating the first term on the right-hand side of Eq. (1), which depends on the hard-collinear coefficients $\mathcal{H}_{\mathrm{NLO}}^{\mathrm{F}}$ and $\mathcal{H}_{\mathrm{NNLO}}^{\mathrm{F}}$, respectively, at NLO and NNLO. The structure of the NLO coefficient $\mathcal{H}_{\mathrm{NLO}}^{\mathrm{F}}$ has been obtained in a universal way from the one-loop corrections to the respective Born subprocess [82]. The general form of $\mathcal{H}_{\mathrm{NNLO}}^{\mathrm{F}}$ is also known [83]: it has been derived from the explicit results for Higgs [84] and vector-boson [85] production in terms of the suitably subtracted two-loop corrections to the respective Born subprocesses. Thus, if the $q \bar{q} \rightarrow F$ (or $g g \rightarrow F$ ) two-loop amplitude is available, the coefficient $\mathcal{H}_{\mathrm{NNLO}}^{\mathrm{F}}$ can be straightforwardly extracted.

\subsection{Implementation within the MATRIX framework}

MATRIX provides a process library for the computation of colour-singlet processes at NNLO QCD. The core of the MATRIX framework is the Monte Carlo program MUNICH, which is capable of computing both QCD and EW [86, 87] corrections to any SM process at NLO accuracy. MUNICH employs an automated implementation of the Catani-Seymour dipole-subtraction method for massless [56, 57] and massive [88] partons, and contains a general implementation of a very efficient, multi-channel based phasespace integration. All amplitudes up to one-loop level are supplied by OPENLOOPS ${ }^{6}[58]$ through an automated interface.

\footnotetext{
6 OPENLOOPS relies on the fast and stable tensor reduction of COLLIER $[89,90]$, supported by a rescue system based on quad-precision CUTTOOLS [91] with ONELOOP [92] to deal with exceptional phasespace points.
} 
With this functionality inherited from MUNICH, MATRIX is immediately able to perform in principle any SM calculation up to NLO accuracy. To promote MUNICH to a Monte Carlo integrator at NNLO QCD, the $F+$ jet cross section at NLO $\left(d \sigma_{\mathrm{NLO}}^{\mathrm{F}+\mathrm{jet}}\right)$ is combined with a process-independent implementation of the $q_{T}$-subtraction formalism for both $g g$ - and $q \bar{q}$-initiated processes within the MATRIX framework. The universal nature of the counterterm $\mathrm{d} \sigma_{\mathrm{NNLO}}^{\mathrm{CT}}$ and the hardcollinear coefficients $\mathcal{H}_{\mathrm{NNLO}}^{\mathrm{F}}$ in Eq. (1) allows us to perform NNLO QCD computations ${ }^{7}$ for the hadroproduction of an arbitrary set of colourless final-state particles, provided that the corresponding two-loop virtual amplitudes for the Bornlevel subprocesses are available.

To this end, MATRIX includes the hard-collinear coefficients of Ref. [84,85], relevant for single Higgs and vectorboson production, and employs own implementations of the two-loop amplitudes for the associated production of a $W / Z$ boson with a photon [60] and $\gamma \gamma$ [94] production, whereas external codes are used for on-shell $Z Z$ [33] and $W^{+} W^{-}$[36] production. ${ }^{8}$ The two-loop amplitudes for offshell production of massive vector-boson pairs[61] are taken from the publicly available code VVAMP [95]. Any new production process of colour singlets can be supplemented to the MATRIX library upon implementation of the corresponding two-loop amplitudes, since all remaining process-dependent ingredients are available in MUNICH + OPENLOOPS and the implementation of the $q_{T}$-subtraction formalism is fully general.

While the idea behind the $q_{T}$-subtraction formalism has been outlined in the previous section, one point deserves some additional discussion. The contribution in the square bracket in Eq. (1) is formally finite in the limit $q_{T} \rightarrow 0$, but both $\mathrm{d} \sigma_{(\mathrm{N}) \mathrm{LO}}^{\mathrm{F}+\mathrm{jet}}$ and $\mathrm{d} \sigma_{(\mathrm{N}) \mathrm{NLO}}^{\mathrm{CT}}$ are separately divergent. Since the subtraction is non-local, we introduce a technical cutoff $r_{\text {cut }}$ on the dimensionless quantity $r=q_{T} / M$ ( $M$ being the invariant mass of the colourless system) which renders both terms separately finite. Below this cut-off, $\mathrm{d} \sigma_{(\mathrm{N}) \mathrm{LO}}^{\mathrm{F}+\mathrm{jet}}$ and $\mathrm{d} \sigma_{(\mathrm{N}) \mathrm{NLO}}^{\mathrm{CT}}$ are assumed to be identical, which is correct up to power-suppressed contributions. The latter vanish in the limit $r_{\text {cut }} \rightarrow 0$ and can be controlled by monitoring the $r_{\text {cut }}$ dependence of the cross section. The absence of any residual logarithmic dependence on $r_{\text {cut }}$ thus provides strong evidence of the correctness of the computation since any mismatch between the contributions would result in a divergence of the cross section for $r_{\text {cut }} \rightarrow 0$. The cut-off on $r$ acts as a slicing parameter, and, correspondingly, the $q_{T}$-subtraction

\footnotetext{
7 On the same basis MATRIX automates also the small- $q_{T}$ resummation of logarithmically enhanced terms at NNLL accuracy (see Ref. [63], and Ref. [93] for more details), which, however, is not yet included in the first release.

${ }^{8}$ Private code provided by T. Gehrmann, A. von Manteuffel and L. Tancredi.
}

method as implemented in MATRIX works very similar to a phase-space slicing method.

To monitor the $r_{\text {cut }}$ dependence without the need of repeated CPU-intensive runs, MATRIX simultaneously computes the cross section at several $r_{\text {cut }}$ values. The numerical information on the $r_{\text {cut }}$ dependence is used to address the limit $r_{\text {cut }} \rightarrow 0$ by using a fit based on the results at finite $r_{\text {cut }}$ values. The extrapolated result, including an estimate of the uncertainty of the extrapolation procedure, is provided at the end of each run. Details on the $r_{\text {cut }} \rightarrow 0$ extrapolation and its uncertainty estimate are presented in Sect. 7, where we also discuss the $r_{\text {cut }}$ dependence of a representative set of the processes available in the first release of MATRIX.

\section{Available processes in MATRIX}

The list of the available processes in MATRIX is provided in Table 1: it includes Higgs boson, vector-boson and vectorboson pair production with all the possible leptonic decay channels of the vector bosons.

\section{How to use Matrix}

The code is engineered in a way that guides the user from the very first execution of MATRIX to the very end of a run of a specific process, obtaining all relevant results. In-between there are certain steps/decisions to make (such as choosing the process, inputs, parameters, ...), which will be described in more detail throughout this and the next section.

The only thing we require the user of MATRIX to provide on the machine where the code is executed is a working installation of LHAPDF, which is a well-known standard code by now, such that lhapdf-config is recognized as a terminal command, or that the path to the lhapdf-config executable is specified in the file MATRIX_configuration (see Sect. 4.5 for more details on the configuration of MATRIX). ${ }^{9}$

\subsection{Compilation and setup of a process}

Assuming that the MATRIX_v1.0.0.tar.gz package is extracted and LHAPDF is installed, the simple command ${ }^{10}$

$\$$. /matrix

executed from the folder MATRIX_V1.0.0 opens the MATRIX shell, an interactive steering interface for the compilation and the setup of a certain process. In principle, one can

\footnotetext{
${ }^{9}$ MATRIX has been tested to work with LHAPDF versions 5 and 6.

10 Note that global compilation settings (if necessary) must be set before starting the code; for options see Sect. 4.5.
} 
Table 1 Available processes in MATRIX

\begin{tabular}{|c|c|c|}
\hline$\$\{$ process_id $\}$ & Process & Description \\
\hline pph21 & $p p / p \bar{p} \rightarrow H$ & On-shell Higgs-boson production \\
\hline ppzo1 & $p p / p \bar{p} \rightarrow Z$ & On-shell $Z$ production \\
\hline ppw01 & $p p / p \bar{p} \rightarrow W^{-}$ & On-shell $W^{-}$production with CKM \\
\hline ppwx01 & $p p / p \bar{p} \rightarrow W^{+}$ & On-shell $W^{+}$production with CKM \\
\hline ppeex02 & $p p / p \bar{p} \rightarrow e^{-} e^{+}$ & $Z$ production with decay \\
\hline ppnenex02 & $p p / p \bar{p} \rightarrow v_{e} \bar{v}_{e}$ & $Z$ production with decay \\
\hline ppenex02 & $p p / p \bar{p} \rightarrow e^{-} \bar{v}_{e}$ & $W^{-}$production with decay and $\mathrm{CKM}$ \\
\hline ppexne02 & $p p / p \bar{p} \rightarrow e^{+} \nu_{e}$ & $W^{+}$production with decay and $\mathrm{CKM}$ \\
\hline ppaa02 & $p p / p \bar{p} \rightarrow \gamma \gamma$ & $\gamma \gamma$ production \\
\hline ppeexa03 & $p p / p \bar{p} \rightarrow e^{-} e^{+} \gamma$ & $Z \gamma$ production with decay \\
\hline ppnenexa03 & $p p / p \bar{p} \rightarrow v_{e} \bar{v}_{e} \gamma$ & $Z \gamma$ production with decay \\
\hline ppenexa03 & $p p / p \bar{p} \rightarrow e^{-} \bar{v}_{e} \gamma$ & $W^{-} \gamma$ with decay \\
\hline ppexnea03 & $p p / p \bar{p} \rightarrow e^{+} v_{e} \gamma$ & $W^{+} \gamma$ with decay \\
\hline ppzz02 & $p p / p \bar{p} \rightarrow Z Z$ & On-shell $Z Z$ production \\
\hline ppwxw02 & $p p / p \bar{p} \rightarrow W^{+} W^{-}$ & On-shell $W^{+} W^{-}$production \\
\hline ppemexmx04 & $p p / p \bar{p} \rightarrow e^{-} \mu^{-} e^{+} \mu^{+}$ & $Z Z$ production with decay \\
\hline ppeeexex0 4 & $p p / p \bar{p} \rightarrow e^{-} e^{-} e^{+} e^{+}$ & $Z Z$ production with decay \\
\hline ppeexnmnmx 04 & $p p / p \bar{p} \rightarrow e^{-} e^{+} v_{\mu} \bar{v}_{\mu}$ & $Z Z$ production with decay \\
\hline ppemxnmnex 04 & $p p / p \bar{p} \rightarrow e^{-} \mu^{+} v_{\mu} \bar{v}_{e}$ & $W^{+} W^{-}$production with decay \\
\hline ppeexnenex 04 & $p p / p \bar{p} \rightarrow e^{-} e^{+} v_{e} \bar{v}_{e}$ & $Z Z / W^{+} W^{-}$production with decay \\
\hline ppemexnmx04 & $p p / p \bar{p} \rightarrow e^{-} \mu^{-} e^{+} \bar{v}_{\mu}$ & $W^{-} Z$ production with decay \\
\hline ppeeexnex04 & $p p / p \bar{p} \rightarrow e^{-} e^{-} e^{+} \bar{v}_{e}$ & $W^{-} Z$ production with decay \\
\hline ppeexmxnm0 4 & $p p / p \bar{p} \rightarrow e^{-} e^{+} \mu^{+} v_{\mu}$ & $W^{+} Z$ production with decay \\
\hline ppeexexne04 & $p p / p \bar{p} \rightarrow e^{-} e^{+} e^{+} v_{e}$ & $W^{+} Z$ production with decay \\
\hline
\end{tabular}

always follow the on-screen instructions; auto-completion of commands should work in all the MATRIX-related shells. The first thing to do is to choose the desired process that should be created and compiled, by typing the respective $\$\{$ process_id\}, e.g.

$$
\mid===>\text { ppz01 }
$$

for on-shell $Z$-Boson production. To find a certain $\$\{$ process_id\}, the command

$$
\mid===>\text { list }
$$

will print a list of all available processes on screen, in the same format as given in Table 1. After entering the process, you will be asked to agree with the terms to use MATRIX. They require you to acknowledge the work of various groups that went into the computation of the present MATRIX process by citing the references provided in the file CITATION.bib. This file is provided with the results in every MATRIX run. In particular, a separate dialog appears for external computations if the implementation of a process is based on them. Simply type

$$
\mid===>Y
$$

for each of these dialogs. Once agreed to the usage terms of MATRIX, the compilation script will automatically pursue the following steps:

- linking to LHAPDF [96];

- download and installation of OPENLOOPS $[58,97]$ (skipped if already installed);

- installation of CLN [98] (skipped if already installed);

- installation of GINAC [98] (skipped if already installed);

- download of the relevant tree-level and one-loop amplitudes through OPENLOOPS (skipped if they already exist);

- compilation of MATRIX process (asked for recompilation if executable exists);

- setting up of the MATRIX process folder under the path run/\$ process_id\}_MATRIX.

Thereafter, the MATRIX shell exits and the process is ready to be run from the created process folder. As instructed on screen, enter that folder,

$$
\text { \$ cd run/\$ process_id\}_MATRIX }
$$

and start a run for this process by continuing with the instructions given in Sect. 4.4. 
We note that a process folder created by MATRIX may be moved to and used from essentially any location on the present machine. Moreover, a MATRIX process folder can be shipped to another system that contains a working installation of the respective process in MATRIX. This requires, however, to change the soft links for bin/run_process and input/MATRIX_configuration inside the process folder to the correct files of the MATRIX installation on the new system.

\subsection{Compilation with arguments}

The MATRIX script also features compilation directly via arguments: Type

\$./matrix --help

in order to see the available options.

We summarize a few useful examples in the following:

1. To directly compile some specific process with ID $\$\{$ process_id\}, simply use the following command:

$\$$./matrix $\$\{$ process_id $\}$

2. To clean the process before compiling (remove object files and executable), add the following option:

\$./matrix \$\{process_id\} --clean_process

3. One can force the code to download the latest OPENLOOPS version even if there is an OPENLOOPS version found on the system, by using

\$./matrix \$\{process_id\} --install_openloops

4. The command

\$./matrix $\$\{$ process_id

--folder_name_extension_my_process_extension

will add an extension to the created process folder such that the default name will be changed to run/\$\{process_id\}_MATRIX_my_process_ extension.

\subsection{General structure of a process folder}

Before providing details on how to actually start the run in a MATRIX process folder, it is useful to understand the essential parts of the general folder structure the code uses and produces while running. This will significantly simplify the comprehension of the code behaviour in the upcoming section. Figure 1 visualizes the general structure: The folders relevant to a user are input, log and result. They will be discussed in detail below, while the others should not be touched/are not of interest (especially for an unexperienced user); the folder bin contains the executable and will only be used to start the MATRIX run shell; the folder default. MATRIX is the default folder for a run of this process, which is copied upon creation of each new run; the run folders denoted by run_XX contain the actual runs started by the user, where XX stands for the name given by the user, or an increasing number starting with 01 in case no name is given (see Sect. 4.4 for more details).

The folders input, log and result all follow the same structure: They contain subfolders of the form run_XX that correspond to each run started by the user, so that the relevant information is kept strictly separated between those different runs. The organization of these subfolders is identical for each run up to differences controlled by the inputs. We note that parts of the folder structure are created in the course of running. Figure 1 shows the folder structure at the very end of a complete run of the most complex type (i.e. including LO, NLO and NNLO with separate PDF choices). In the following we discuss the purpose and the organization of the relevant folders for such a run:

- input:

- Three different cards can be modified in order to adjust all the run settings (of physics-related and technical kind), model parameters and distributions to be generated in the run. The respective files can be accessed directly or through the interface of the MATRIX run shell; see Sect. 5 for details on the input cards.

* The file parameter.dat controls the physics-related run settings, such as collider type, machine energy, PDFs, etc., but also technical parameters, such as which orders in perturbation theory should be computed, which precision is to be achieved in the run, if distributions are computed, if the loop-induced $g g$ contribution is included, etc.

* The file model. dat sets all relevant model parameters, such as masses, widths, etc.

* The file distribution. dat gives the possibility to define distributions from the final-state particles with certain ranges, bin sizes, etc. (only effective if distributions are turned on in the file parameter.dat).

- The process-specific fileMATRIX_configuration for general MATRIX configurations inside the folder input is the same for all runs of this process and can be modified to use an individual configuration for this process (by default it is a soft link to the global file MATRIX_configuration inside the folder MATRIX_V1.0.0/config, but may be replaced by a copy of this file, see Sect. 4.5)

- log:

- This folder is for debugging purposes only. Log files $\left({ }^{*} . \log\right.$ files) are saved for every single job that is 


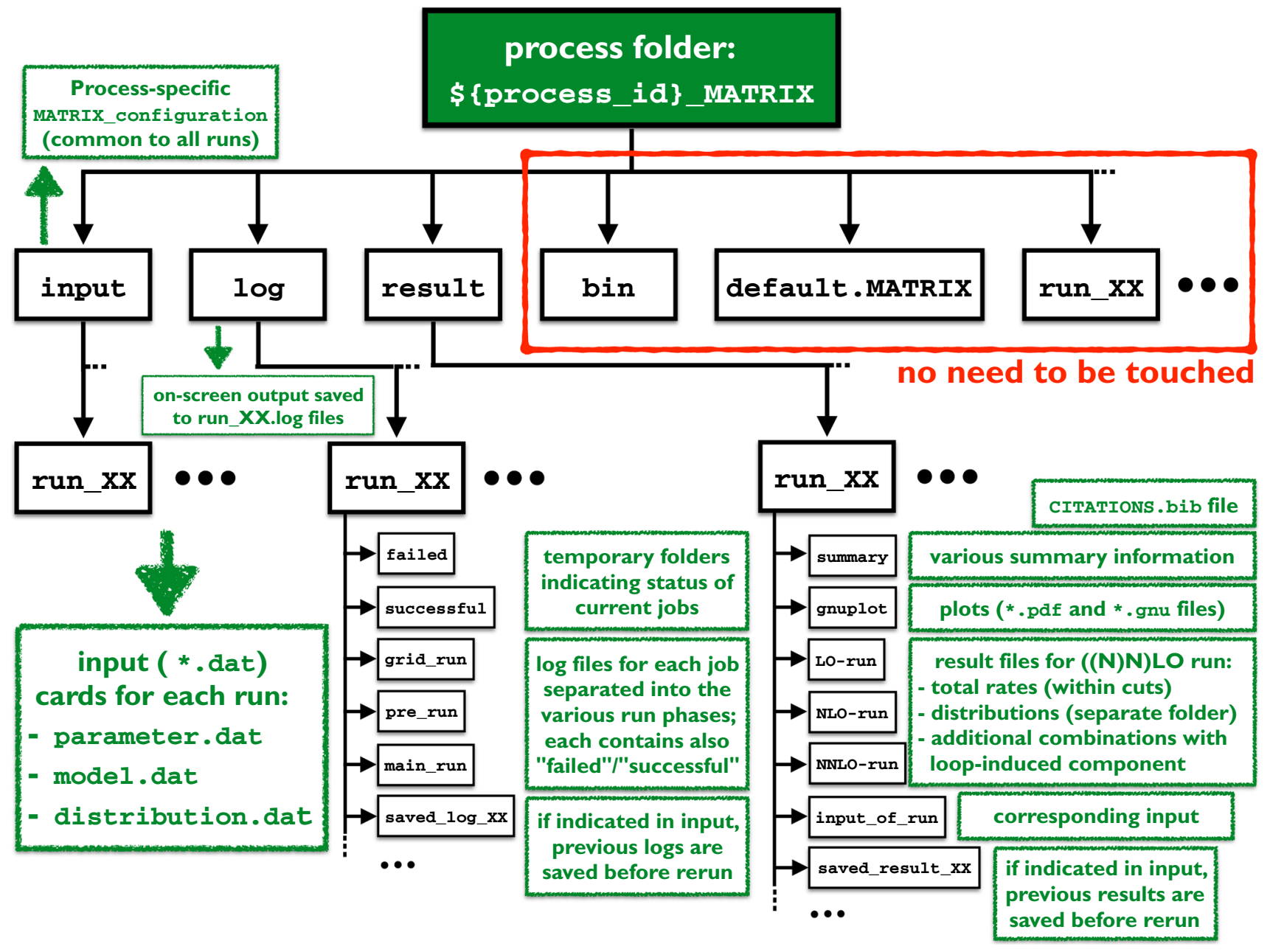

Fig. 1 Overview of the folder structure inside a MATRIX process folder

started during a run. Once a job has finished successfully, this is indicated by a file created in the successful folder. If a job fails (even after a certain number of retries) a corresponding file will be added to the failed folder.

- At the end of each running phase (grid-run, pre-run, main-run; see Sect. 4.4.1) the respective $\log$ files (including the successful and failed folders) are moved into the folders grid_run, pre_run and main_run, respectively.

- If an existing run, which has already created log files in the respective log folder, is picked up and started again, those $\log$ files are saved into a subfolder saved_log_XX, where XX is an increasing number starting at 01 (only working if the respective switch in the file parameter. dat is turned on; default: turned off).

- The on-screen output of the MATRIX run script is saved for each run to a file run_XX. Iog.
- result:

- This folder contains all relevant results that are generated during and collected at the end of a run.

- A file CITATIONS.bib is created with every run, which contains the citation keys for all publications that were relevant for the specific run. Please cite these papers if you use the results of MATRIX to acknowledge the efforts that have been made to obtain these results with MATRIX.

- The folder summary contains information on the respective run. In particular, the summary of all total rates (possibly within cuts), which are also printed on screen at the end of each run, is saved to the file result_summary.dat (currently the only file there).

- In the folder gnuplot one finds (automatically generated) * . gnu and *. pdf files for every distribution created during the run. Its histogram subfolder contains the data prepared and used for these plots. 
Additionally, all pdf files are combined into a single file al1_plots.pdf using the pdfuni te binary. If either gnuplot or pafuni te do not exist on the system, the corresponding *.paf files are not created.

- The total rates and distributions are saved to plain text files in the folders $\mathrm{LO}$-run, $\mathrm{NLO}$-run and NNLO-run. This separation reflects the different PDF sets that can be chosen for each of the three runs (in the file parameter.dat; see Sect. 5.1.1.3). Total rates (possibly within cuts) are saved to the files rate_XX.dat (including scale variations if turned on in the file parameter.dat; see Sect. 5.1.1.2), where, depending on the considered order, XX can be LO, NLO_QCD, NNLO_QCD and loop-induced _QCD. Additional files rate_extrapolated_XX are created for total rates, which are computed with the $q_{T}$-subtraction method (NNLO, and possibly NLO): They provide extrapolated results for $r_{\text {cut }} \rightarrow 0$ as the final results, see Sect. 7, while the original rate files contain only the cross sections calculated at a finite $r_{\text {cut }}$ value. Inside each of the * -run folders the distributions are saved to a folder distributions (including minimum and maximum results of the scale variations). There is an extra distribution folder distributions_NLO_plus_loop-induced inside the folder NLO-run, which contains the results of the NLO distributions combined with the loop-induced contribution (if turned on). Besides, there are folders distributions_NLO_prime _plus_loop-induced and distributions _only_loop-induced inside the folder NNLO - run which contain the combined $\mathrm{NLO}^{\prime}+g g$ contribution $^{11}$ and the pure $g g$ contribution, respectively.

- The folder input_of_run contains the three input cards (parameter.dat, model.dat, distri bution. dat), which were copied at the beginning of the respective run.

- If an existing run, which has already created results in the respective result folder, is picked up and started again, those results are saved into a subfolder saved_result_XX, where $\mathrm{XX}$ is an increasing number, starting with 01 (only working if the respective switch in the file parameter. dat is turned on; default: turned on).

$\overline{11} \mathrm{NLO}^{\prime}+g g$ refers to the sum of the NLO and $g g$ contributions, where both are computed with NNLO PDFs. This can generally be regarded as the best prediction before the full NNLO became available.

\subsection{Running a process}

\subsubsection{Running with interactive shell}

From the MATRIX process folder (default: run/\$ proce ss_id\}_MATRIX) the command ${ }^{12}$

\$ . / bin/run_process

opens the MATRIX run shell, an interactive steering interface for handling all run-related settings, inputs and options. ${ }^{13}$ From here on one can simply follow the on-screen instructions of the MATRIX run shell; we thus only summarize the most relevant steps in the following.

First, one must choose a name,

$\mid===>$ \$ $\{$ run_name $\}$

for the run, which has to begin with run_, to generate a new run. Alternatively, one can also list and choose one of the runs which already exist (have been created before). As in all MATRIX shells, auto-complete should work here. The general idea is that each run is separate, i.e. each of these runs will create its own run folder ( $\$$ run_name $\})$ and the corresponding subfolders inside input, log and result. An old run can only be picked up when the previous one is not running any more. One should, however, be careful with this option since all data of the old run will be overwritten (except for possibly the results and the log files, see Sect. 4.3).

Next, we can choose from a list of several commands printed on screen. These commands are divided into three groups: general commands, input to modify, run modes. Information on each individual command ( $\$$ command $\}$ ) can be received through the help menu by typing

$\mid===>$ help $\$\{$ command $\}$

In order to directly modify the input cards from the shell (opened in the default editor ${ }^{14}$ ), one can simply type the name of the input file

$$
\mid===>\text { \$ }\{\text { name_input_file }\}
$$

where $\$\{$ name_input_file\} can be either para meter, model or distribution. Changes will be done directly to the respective files parameter.dat, model.dat or distribution. dat inside the folder input/\$\{run_name\} (see Sect. 4.3). Details on the

\footnotetext{
12 Note that the global configuration (if necessary) must be set in the file input/MATRIX_configuration before starting the run; see Sect. 4.5 for a description of the general options.

13 The script can also be started with certain arguments, see Sect. 4.4.2.

14 The default editor can be set through the default_editor variable of the file MATRIX_configuration, or by exporting directly the EDITOR environment variable on the system, e.g. export $E D I T O R=e m a c s$, where the respective editor (here: emacs) must be installed and recognized as a terminal command.
} 
impact of the various parameters, which can be accessed through the input files, are described in Sect. 5. ${ }^{15}$

After adjusting the input cards to obtain the desired results, we can start the run by typing

$$
\text { |===> run }
$$

This will start a complete run, no human intervention is needed from now on. Once the run is finished the results from the run are collected in the respective folder result/\$ \{run_name $\}$ as printed on screen (see Sect. 4.3 for details on the result-folder structure), the most relevant results, which are the total rates, are also printed on screen at the very end of the run. ${ }^{16}$ We emphasize that for every run a CITATION.bib file is created and provided inside the folder result/\$\{run_name\}. Please cite these papers if you use the results of MATRIX to acknowledge the efforts that have been made to obtain these results with MATRIX.

When performing a time-extensive (NNLO) run, we recommend to start MATRIX from a window manager (e.g. screen or tmux) in order to be able to logout from the present machine during the run. An alternative is to start MATRIX with nohup as explained in the second example of Sect. 4.4.2.

\section{Running phases of a complete run}

A complete run is divided into various stages (running phases), each of which may be started directly from the run shell by typing the name of the respective run mode ( $\$\{$ run_mode $\}$ ). One must bear in mind, however, that every run stage depends on all previous run stages and will fail in case one of the previous ones has not finished successfully. The order of the run stages is as follows:

- grid-run: First, the integration grids are created in the warm-up phase (run_grid).

- pre-run: Next, the expected runtimes for the main-run are extrapolated from a quick pre-run phase (run_pre); some preliminary results are already printed on screen.

- main-run: Then, the main run is started, computing all results to the desired precision (run_main).

- result-collection: Finally, the results are collected, and all distributions are automatically plotted if gnuplot is installed (run_results).

\footnotetext{
15 By default the inputs are already set to use reasonable cuts and parameters for each process; the default run (without changing the cards) computes a simple LO cross section with $1 \%$ precision, which we recommend to use when running for the first time in order to test whether everything is working properly. As this run should be very quick (a few minutes), this test can be done in local mode (see Sect. 4.5 for the settings in the file input/MATRIX_configuration).

16 Note that MATRIX provides the extrapolated cross section for $r_{\text {cut }} \rightarrow$ 0 as a final result at NNLO (and at NLO if the $q_{T}$-subtraction procedure is used also at NLO) including an extrapolation uncertainty, see Sect. 7, which is printed on screen after the cross section with a fixed $r_{\text {cut }}$ value.
}

Note that the result-collection will always be started automatically after a successful main-run. Furthermore, if the run mode run_pre_and_main is used, the code will start from the pre-run (assuming a successful grid-run) and automatically continue with the main-run and result-collection.

Starting from one of these intermediate stages can be useful in many respects. One example is the continuation of a run after some unwanted behaviour, if some stages have already passed successfully and one would like to restart from one of the later stages. Note that all jobs in the requested run stage are removed and started from scratch. To continue a run while keeping already successfully finished jobs of the requested run stage, or to run with increased precision, the --cont inue command can be used, see example seven of Sect. 4.4.2. Another example is running again with a modified set of inputs. In the latter case it is sufficient to only restart the main-run as follows:

$\$$./bin/run_process

to start the script,

$\mid===>$ \$ $\{$ run_name $\}$

to pick up the old run with name $\$\{$ run_name $\}$, and

$$
\mid===>\text { parameter }
$$

to change, e.g., the PDF set in the file parameter. dat (if not done by hand before). It is essential to also uncomment include_pre_in_results $=0$ in the same file in order to avoid mixing of the different settings in pre-run and main-run in the result combination. After that, the mainrun is started by

$$
\text { |===>> run_main }
$$

Other run modes to be selected involve different behaviour of the code, such as only setting up the folder \$ \{run_name \} and the corresponding subfolders inside input, log and result (setup_run) without starting the run (this is helpful if one wants to change the inputs by hand, but not through the interface, e.g. by copying the input files from somewhere else); deleting a given run including its respective subfolders inside input, log and result (delete_run); etc.

\subsubsection{Running with arguments}

The run script allows some of the various settings, which are typically controlled interactively, to be controlled directly by arguments in its shell command. This enables, e.g., the possibility to directly start a certain run without having to interact with the interface. Type

$$
\text { \$ . /bin/run_process --help }
$$

in order to see all available options. 
We summarize a few useful examples in the following:

1. The command

$$
\begin{aligned}
& \text { \$./bin/run_process } \$\left\{r u n \_n a m e\right\} \\
& \text {--run_mode run }
\end{aligned}
$$

will create (pick up, if $\$\left\{r u n \_n a m e\right\}$ exists) the run with name $\$\left\{r u n \_n a m e\right\}$, and directly start a complete run (due to--run_mode run), with the default inputs (or the ones already set in $\$\{$ run_name $\}$ ). ${ }^{17}$ The $\$\left\{r u n \_m o d e\right\}$ may be chosen as any of the various commands outlined at the end of the previous section, e.g. --run_mode run_pre_and_main to start the run directly from the pre-run (assuming a successful gridrun has already been done).

2. The same command can be used in combination with nohup

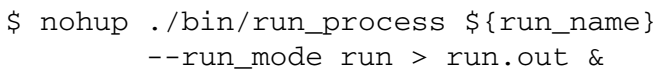

to run MATRIX in the background while one is still able to logout from the present machine. The on-screen output of MATRIX in this example is written to the file run. out.

3. The command

$$
\begin{aligned}
& \text { \$./bin/run_process } \$\{\text { run_name } \\
& \text {--delete_run }
\end{aligned}
$$

will delete the run with name $\$\{$ run_name\}, including its respective subfolders inside input, log and result.

4. The command

$$
\text { \$./bin/run_process \$\{run_name }\} \text {--setup_run }
$$

will create a run with name $\$\{$ run_name $\}$ including its respective subfolders inside input, log and result without starting the run. One may then modify the input files directly by hand (without using the MATRIX shell) and continue with starting the run as described under 1.).

5. One may want to copy, e.g. as a backup, some existing (possibly finished) run. The command

$$
\begin{aligned}
& \$ . / \text { bin/run_process } \$\left\{r u n \_n a m e\right\} \\
& - \text {-copy_run_from } \$\left\{r u n \_a n o t h e r \_n a m e\right\}
\end{aligned}
$$

allows to make a complete copy of an existing run with name $\$\left\{r u n \_a n o t h e r \_n a m e\right\}$ to a new run with name $\$\{$ run_name $\}$. This may take quite a while in case a finished run is copied, as the run folder could have a rather large size.

6. In certain situations it may be helpful to use inputs other than the default inputs when creating a new run. The command

$$
\begin{aligned}
& \$ \text {./bin/run_process } \$\left\{r u n \_n a m e\right\} \\
& \text {--input_dir } \$\{\text { any_folder_inside_input }\}
\end{aligned}
$$

\footnotetext{
${ }^{17}$ Note that in the default inputs only a simple LO run is enabled.
}

will create a run with name $\$\{$ run_name $\}$, and the three input files will be copied from the folder \$ $\left\{a n y \_f o l d e r \_i n s i d e \_i n p u t\right\}$ inside the folder input. This may, of course, also be the name of another run whose inputs should be used. The only requirement is that a folder with the given name exists inside the folder input and contains the files parameter. dat, model. dat and distribution. dat.

7. MATRIX provides the possibility to continue a run, while deleting only the content of later run stages, but not of the current run stage. This is very useful in two situations: First, a run has crashed in the middle or at the end of a run stage, but several jobs have already finished successfully. Second, the precision of a run should be improved by adding more statistics to a previous run. In both cases the command

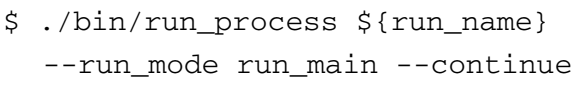

will continue the run with name $\$\left\{r u n \_n a m e\right\}$ and not delete any job that has already finished successfully. Note that it is absolutely required not to change any of the inputs, except for a possibly increased precision, with respect to the previous run if the flag--continue is used. Any other $\$\{$ run_mode $\}$ may be chosen to be continued in this way.

\subsection{Configuration file}

Before turning to physics-related and technical settings relevant for a specific MATRIX run in Sect. 5, we discuss the global parameters that steer the general behaviour of the code. The file MATRIX_configuration controls various global settings for both the compilation and the running of the code. The general idea is that these configurations can be made once and for all, depending on the respective environment one is working on: one can, e.g., set the relevant paths for the compilation (if not found automatically), choose local running or specify the cluster scheduler available on the present machine, etc. The global settings that affect the running of a process may still be altered at a later stage (before starting the respective run) and can be chosen different for different process folders. The main file MATRIX_configuration can be found in the folder config inside the MATRIX main folder. This file is linked during each setup of a process (see Sect. 4.1) into the folder input of the respective process folder. This soft link may be replaced by the actual file such that each process can have its own configuration file. This allows for process-specific run settings, and one can, e.g., change from cluster to local 
Table 2 Parameters to be set in the file MATRIX_configuration

\begin{tabular}{|c|c|}
\hline Variable & Description \\
\hline default_editor & $\begin{array}{l}\text { Sets the editor to be used for interactive access to input files. Alternatively, the default editor may } \\
\text { be configured directly by exporting the EDITOR environment variable on the system }\end{array}$ \\
\hline mode & Switch to choose local (multicore) run mode or cluster mode \\
\hline cluster_name & $\begin{array}{l}\text { Name of the cluster; currently supported: Slurm, LSF (e.g. lxplus), Condor, HTCondor (e.g. } \\
\text { lxplus), PBS, Torque, SGE. }\end{array}$ \\
\hline cluster_queue & Queue/Partition of the cluster to be used for cluster submit; not required in most cases \\
\hline cluster_runtime & Runtime of jobs in cluster submit; not required in most cases \\
\hline cluster_submit_line[1-99] & Lines in cluster submit file to add cluster-specific options \\
\hline max_nr_parallel_jobs & Number of cores to be used in multicore mode; maximal number of available cores on cluster \\
\hline parallel_job_limit & Upper threshold for number of parallel jobs; if exceeded, user intervention required to continue. \\
\hline max_jobs_in_cluster_queue & $\begin{array}{l}\text { If cluster queue contains more jobs than this value, MATRIX will wait until jobs finish before } \\
\text { submitting further jobs }\end{array}$ \\
\hline path_to_executable & $\begin{array}{l}\text { This path can be set to the folder that contains the executables of the processes (usually bin in the } \\
\text { MATRIX main folder), and provides the possibility to use an executable from a different MATRIX } \\
\text { installation; not required in most cases }\end{array}$ \\
\hline max_restarts & $\begin{array}{l}\text { If there are still jobs left that failed after all jobs finished, MATRIX will restart all failed jobs } n \text { times } \\
\text { when this parameter is set to } n\end{array}$ \\
\hline nr_cores & $\begin{array}{l}\text { Number of cores to be used for the compilation; determined automatically by the number of } \\
\text { available cores on the machine if not set }\end{array}$ \\
\hline path_to_lhapdf & Path to lhapdf-config; not required in most cases \\
\hline path_to_openloops & Path to the openloops executable; not required in most cases \\
\hline path_to_ginac & Path to the ginac installation; not required in most cases \\
\hline path_to_cln & Path to the $c \ln$ installation; not required in most cases \\
\hline path_to_libgfortran & $\begin{array}{l}\text { Path to the libg fortran library; not required in most cases. This path can also be used if the } \\
\text { libquadmath library is not found, to be set to the respective } 1 \mathrm{ib} \text { folder }\end{array}$ \\
\hline path_to_gsl & Path to gsl-config; not required in most cases \\
\hline
\end{tabular}

run mode for a specific process (or even only for a dedicated run and change it back after having started the run). ${ }^{18}$

The options controlled by the file MATRIX_configu ration are listed in Table 2.

\section{Settings of a MATRIX run}

In this section all relevant input settings are discussed. Most of them are directly physics-related, but there are also a few more technical parameters.

\subsection{Process-independent settings}

Every run of a process contains three input files in its respective subfolder inside input, which can be modified by the user. The generic inputs in the files parameter.dat, model. dat and distribution. dat of each MATRIX run are described in the following.

\footnotetext{
18 Since the file MATRIX_configuration is read only at the beginning of a run, any change done after that has no effect.
}

\subsubsection{Settings in parameter. dat}

All main parameters, related to the run itself or the behaviour of the code, are specified in the file parameter. dat. Most of them should be completely self-explanatory, and we will focus our discussion on the essential ones. The settings can be organized into certain groups and are discussed in the order they appear in the file parameter. dat for the sample case of $Z \gamma$ production (where applicable).

\subsubsection{General run settings}

\begin{tabular}{|llll|}
\hline process_class & $=$ pp-emepa+X & $\#$ & process id \\
E & $=6500$. & $\#$ energy per beam \\
coll_choice & $=1$ & $\#$ & (1) PP collider \\
& & & (2) PPbar collider
\end{tabular}

process_class A unique identifier for the process under consideration; it should never be touched by the user; in particular, no other process can be chosen at this stage. Its sole purpose is to identify which process the respective parameter file belongs to.

E Value of the energy per beam; assumed to be identical for both initial hadrons, i.e. equal to half of the collider energy. Here and in what follows, all input parameters with energy dimension are understood in units of $\mathrm{GeV}$. 


\subsubsection{Scale settings}

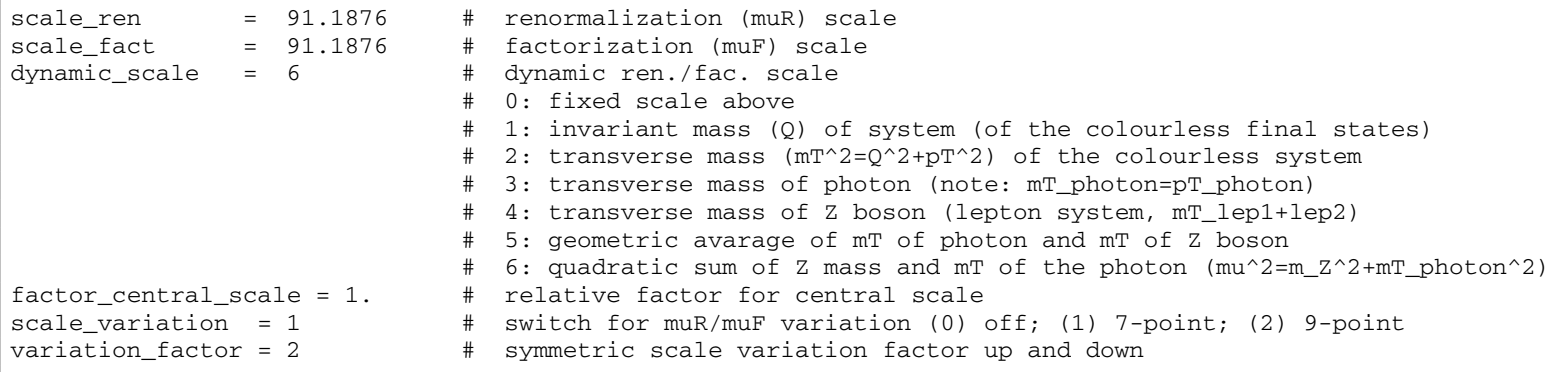

dynamic_scale This parameter allows the user to choose between the specified fixed renormalization and factorization scales (scale_ren/scale_fact) and dynamic ones. A dynamic scale must be implemented individually for the process under consideration. For all processes two dynamic scales are provided by default: the invari- variation_factor This (integer) value determines by which factor with respect to the central scale the scale variation is performed.

\subsubsection{Order-dependent run settings}

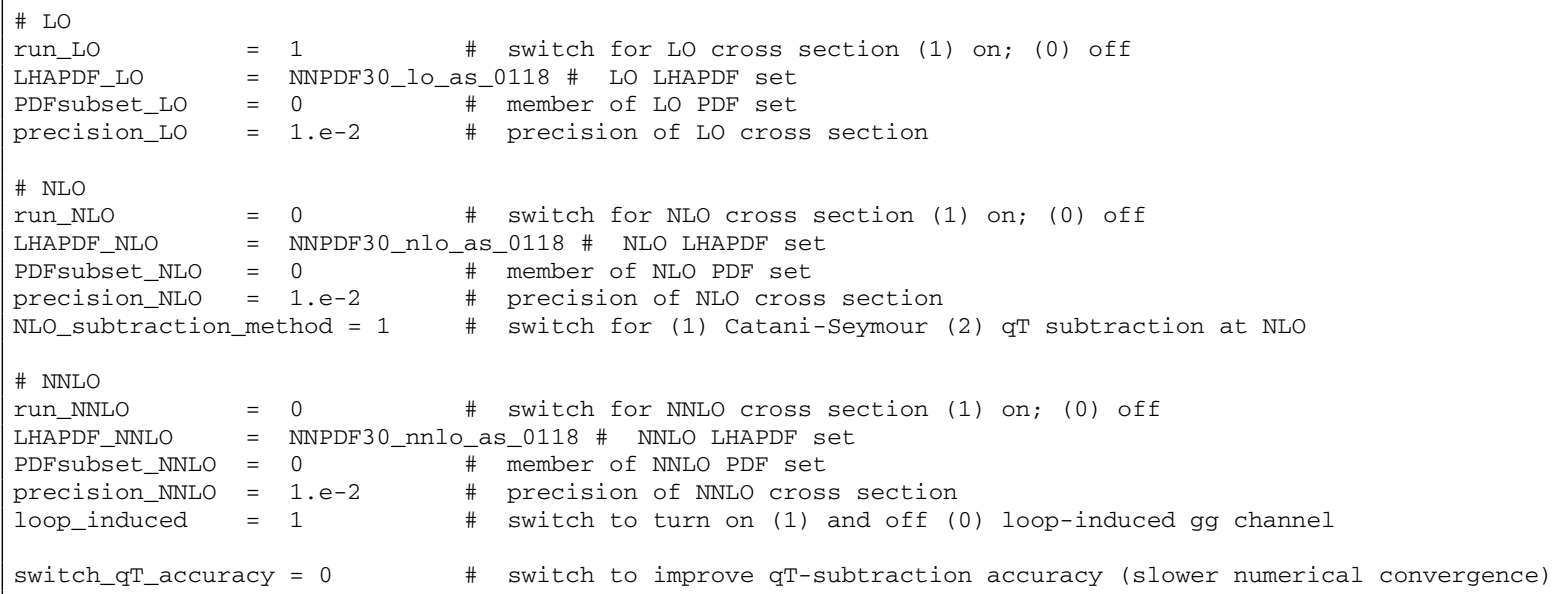

ant mass (dynamic_scale $=1$ ) and the transverse mass (dynamic_scale $=2$ ) of the colourless final-state system. The relevant file of the $\mathrm{C}++$ code is prc/\$process_id\}/user/specify.scales. CXX in the MATRIX main folder (recompilation needed if modified!). All additional dynamic scale choices for each process are discussed in Sect. 5.2. A user interested in setting a specific dynamic scale which has not been implemented yet for this process is advised to contact the authors. ${ }^{19}$

factor_central_scale A relative factor that multiplies the central scale; particularly useful for dynamic scales.

\footnotetext{
19 A short description on how to add user-specified scales, cuts and distributions to the $\mathrm{C}++$ code is given in Appendix $\mathrm{B}$ for the advanced user.
}

A single run of a process in MATRIX involves up to three different orders ( $\$\{$ order $\}$ ), namely LO, NLO and NNLO. For each of these orders we may choose the following inputs:

run_\$ $\{$ order $\}$ Switch to turn on and off the order $\$\{$ order $\}$ in the run.

LHAPDF_\$ $\{$ order $\}$ LHAPDF string that determines the PDF set used at this order with the respective member PDFsubset_\$ $\{$ order\}.

precision_\$ $\{$ order $\}$ Desired numerical precision of the cross section (within cuts) of this run.

NLO_subtraction_method Switch to choose the NLO subtraction scheme: For the NLO part of the computation two different subtraction schemes are available. The default is the Catani-Seymour dipole subtraction, which comes with the advantage of being fully local and thus does 
not lead to any $r_{\text {cut }}$ dependence. The NLO computation can also be performed by means of the $q_{T}$-subtraction method. The option to use both subtraction schemes in the same run is currently not supported.

loop_induced For certain processes (such as $Z Z$, $\left.W^{+} W^{-}, \ldots\right)$ a loop-induced $g g$ contribution enters at the NNLO; this contribution is separately finite and can be included or excluded by this switch; if a process has no loopinduced $g g$ component, the switch is absent.

switch_qT_accuracy Switch specific to processes with large $r_{\text {cut }}$ dependence (in particular processes with finalstate photons). The lowest calculated value of $r_{\text {cut }}$ is changed from $r_{\text {cut }}=0.15 \%$ (switch_qT_accuracy $=0$ ) to $r_{\text {cut }}=0.05 \%$ (switch_qT_accuracy $\left.=1\right)$ in order to improve the accuracy of the $q_{T}$-subtracted NNLO cross section, at the cost of numerical convergence. We refer to Sect. 5.2 for further information.

\subsubsection{Settings for fiducial cuts}

We first note that certain settings, such as photon isolation, naturally only affect dedicated processes. The default input files are adapted such that they only contain options that are of relevance for the respective process. It is not recommended to add any new blocks to the input files in order to avoid unwanted behaviour, although such additional settings would usually just not have any impact on the run.

\section{Jet algorithm}

$$
\Delta R_{j j}=\sqrt{\Delta \eta_{j j}^{2}+\Delta \phi_{j j}^{2}} \quad \text { or } \quad \Delta R_{j j}=\sqrt{\Delta y_{j j}^{2}+\Delta \phi_{j j}^{2}}
$$

jet_R Value of the jet radius used for the jet definition.

This sets the relevant parameters for the jet algorithm. Selection cuts on jets, including the setting for their minimal transverse momenta and maximal (pseudo-)rapidity, are described below under the paragraph Particle definition and generic cuts.

\section{Photon isolation}

For all processes involving identified final-state photons, MATRIX relies on the smooth-cone photon isolation procedure from Ref. [103], which works as follows: For every cone of radius $\delta=\sqrt{(\Delta \eta)^{2}+(\Delta \phi)^{2}}<\delta_{0}$ around a final-state photon, the total amount of hadronic (partonic) transverse energy $E_{T}$ inside the cone has to be smaller than $E_{T}^{\max }(\delta)$,

$$
\begin{gathered}
\sum_{i=\text { hadrons(partons) }} p_{T, i} \Theta\left(\delta-\delta_{i \gamma}\right) \leq E_{T}^{\max }(\delta) \\
=E_{T}^{\mathrm{ref}}\left(\frac{1-\cos \delta}{1-\cos \delta_{0}}\right)^{n} \quad \forall \delta \leq \delta_{0}
\end{gathered}
$$

where $E_{T}^{\text {ref }}$ is a reference transverse-momentum scale that can be chosen to be either a fraction $\epsilon_{\gamma}$ of the transverse momentum of the respective photon $\left(p_{T, \gamma}\right)$ or a fixed value $\left(p_{T}^{0}\right)$

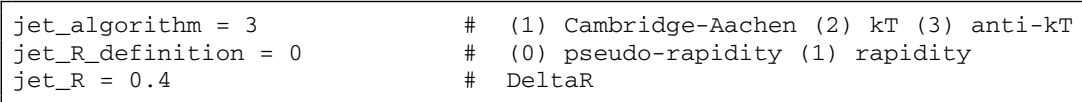

jet_algorithm Switch to choose between three predefined jet-clustering algorithms: Cambridge-Aachen [99, $100], k_{T}[101]$ or anti- $k_{T}[102] .^{20}$

$$
E_{T}^{\mathrm{ref}}=\epsilon_{\gamma} p_{T, \gamma} \quad \text { or } \quad E_{T}^{\mathrm{ref}}=p_{T}^{0} .
$$

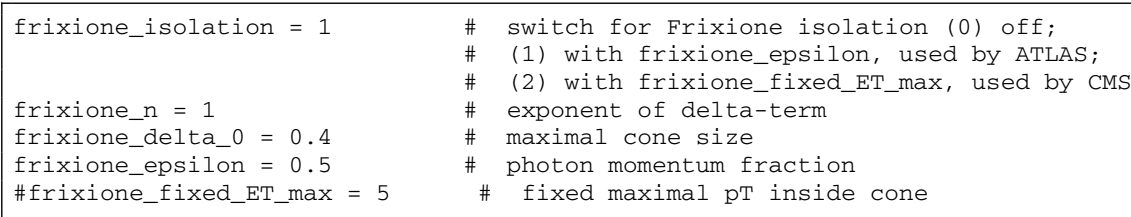

jet_R_definition According to the setting of this switch, the distance $\Delta R$ of jets is defined either via pseudorapidity or rapidity,

\footnotetext{
${ }^{20}$ We note that, for the processes considered in the first release of MATRIX, the three algorithms are actually equivalent, since the final state contains at most two partons. Also parameter jet_R_ definition has no impact for final states with at most two partons, as the pseudo-rapidity and rapidity of massless partons is identical.
}

frixione_isolation Switch for smooth-cone photon isolation with three possible settings: turned off; using one or the other alternative in Eq. (4).

frixione_n Value of $n$ in Eq. (3).

frixione_delta_0 Value of $\delta_{0}$ in Eq. (3).

frixione_epsilon Value of $\varepsilon_{\gamma}$ in Eq.(4). Only used for frixione_isolation $=1$, and must be commented if frixione_isolation $=2$. 
frixione_fixed_ET_max Value of $p_{T}^{0}$ in Eq.(4). Only used for frixione_isolation $=2$, and must be commented if frixione_isolation $=1$.

Selection cuts on photons, including the setting for their minimal transverse momenta and maximal (pseudo-)rapidity, are described in the following paragraph.

\section{Particle definition and generic cuts}

Some fiducial cuts are defined in a general, i.e. processindependent, way by requiring a minimum and maximum multiplicity of a certain (group of) particle(s) with given requirements (such as minimal transverse momentum or maximal rapidity). For that purpose, the user can define which requirements (clustered) parton-level objects need to fulfil in order to be considered particles that can be accessed in scale definitions, phase-space cuts and distributions. Table 3 summarizes the content of all relevant predefined particle groups. All objects entering these groups will be ordered by their transverse momenta, starting with the hardest one.

The parameters define_y $\$\{$ particle_group and define_eta $\$\{$ particle_group $\}$ set the geometric range for the acceptance of particles in $\$\{$ particle_group\}, in terms of upper limits on the absolute value of rapidity and/or pseudo-rapidity, respectively, in the hadronic frame. Objects that do not fulfil these requirements are discarded in the respective particle group. For example, define_eta lepton $=2.5$ defines all leptons in the respective group with a maximal absolute pseudo-rapidity of 2.5 .

The parameter define_pT \$\{particle_group\} sets a threshold on the transverse momentum of particles in \$ particle_group\}. Objects below that threshold are not discarded, but they do not increase the multiplicity counter of accepted particles in the respective $\$\left\{\right.$ particle_group\}. They enter the respective $\left(p_{T^{-}}\right.$ ordered) particle groups at the very end of the group.

Setting only the above parameters does not result in selection cuts yet. To define requirements on the multiplicity counter of accepted particles of that $\$$ particle_group $\}$, the parameters n_observed_min \$\{particle_ group \}, and n_observed_max $\$$ particle_group are used: They define how many particles of that group must be observed at least and at most, respectively, in the final state for an event to be accepted. No cut is applied here if the minimum and maximum requirements do not impose an actual restriction.

These parameters are organized in blocks for each $\$\{$ particle_group $\}$ in the file parameter. dat with the following general structure:

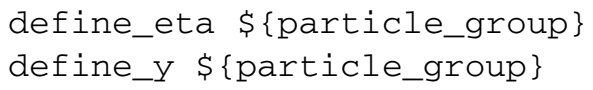

Table 3 All relevant particle groups predefined in MATRIX. Each group is ordered by the transverse momenta of the respective particles, starting with the hardest one. These groups are most important to recognize by the user in two situations: when using the predefined blocks for fiducial cuts and when defining distributions (see Sect. 5.1.3). Furthermore, they can be accessed directly in the $\mathrm{C}++$ code which is essential to the advanced user when defining user-specified scales, cuts and distributions, see Appendix B

\begin{tabular}{|c|c|}
\hline Identifier & Description \\
\hline jet & $\begin{array}{l}\text { Parton-level jets, } 5 \text { light quarks+gluons, clustered } \\
\text { according to jet algorithm }\end{array}$ \\
\hline ljet & Light jets: same as jet, but without bottom jets \\
\hline bjet & Bottom jets: jets with a bottom charge (see main text) \\
\hline photon & $\begin{array}{l}\text { Photons, isolated according to chosen smooth-cone } \\
\text { isolation }\end{array}$ \\
\hline lep & $\begin{array}{l}\text { Charged leptons, i.e. electrons and muons, including } \\
\text { particles and anti-particles }\end{array}$ \\
\hline $\operatorname{lm}$ & Negatively charged leptons, i.e. electrons and muons \\
\hline $1 \mathrm{p}$ & $\begin{array}{l}\text { Positively charged leptons, i.e. positrons and } \\
\text { anti-muons }\end{array}$ \\
\hline e & Electrons and positrons \\
\hline em & Electrons \\
\hline ep & Positrons \\
\hline $\mathrm{mu}$ & Muons and anti-muons \\
\hline mum & Muons \\
\hline mup & Anti-muons \\
\hline z & $\mathrm{Z}$ bosons \\
\hline $\mathrm{w}$ & $W^{+}$and $W^{-}$bosons \\
\hline wp & $W^{+}$bosons \\
\hline wm & $W^{-}$bosons \\
\hline $\mathrm{h}$ & Higgs bosons \\
\hline nua & Neutrinos and anti-neutrinos \\
\hline nu & Neutrinos \\
\hline nux & Anti-neutrinos \\
\hline nea & Electron-neutrinos and anti-electron-neutrinos \\
\hline ne & Electron-neutrinos \\
\hline nex & Anti-electron-neutrinos \\
\hline nma & Muon-neutrinos and anti-muon-neutrinos \\
\hline $\mathrm{nm}$ & Muon-neutrinos \\
\hline $\mathrm{nmx}$ & Anti-muon-neutrinos \\
\hline missing & $\begin{array}{l}\text { Sum of all neutrino momenta, containing only one } \\
\text { entry (special group) }\end{array}$ \\
\hline
\end{tabular}

$$
\begin{aligned}
& \text { define_pT } \$\{\text { particle_group }\} \\
& \text { n_observed_min } \$\{\text { particle_group }\} \\
& \text { n_observed_max } \$\{\text { particle_group }\}
\end{aligned}
$$

Such blocks are predefined for the relevant particle groups of each process in the respective file parameter. dat. They should be sufficient for most practical purposes, and it is generally recommended to stick to the predefined blocks. Nevertheless, it is possible to add additional blocks also for the other particle groups using the structure above. In this case, care has to be taken to avoid unwanted behaviour. In 
particular, requiring a certain number of particles which actually do not exist in the final state of a given process must

\section{Lepton cuts}

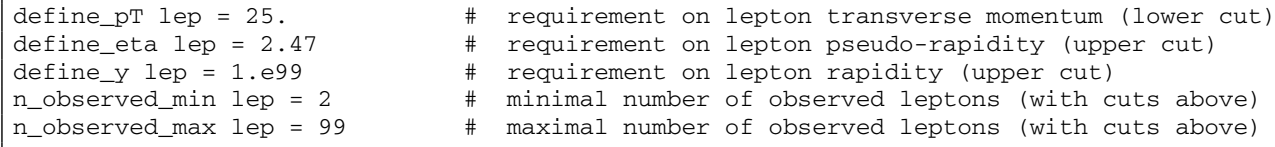

be avoided. Below, we provide examples of the respective blocks available in various processes.

Jet cuts
This block defines each lepton in the particle group lep to have a minimal transverse momentum of $25 \mathrm{GeV}$ and a maximal absolute pseudo-rapidity of 2.47 . It further requires the

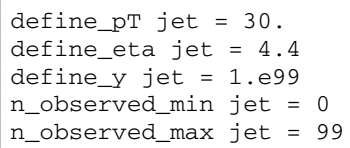

This defines the particle group jet with a minimal transverse momentum of $30 \mathrm{GeV}$ and a maximal absolute pseudorapidity of 4.4 , using the jet-clustering algorithm specified above. No phase-space cut is effective, since this process has a maximum of two jets in the final state at NNLO, and neither a minimal $(>0)$ nor a maximal number $(<2)$ of observed jets is required. ${ }^{21}$ However, the particle group jet with the presence of at least two such leptons. All events not passing this criterion are discarded from the fiducial phase space. ${ }^{23}$

Analogous blocks are available for other particle groups of charged leptons, namely $1 \mathrm{~m}, 1 \mathrm{p}, \mathrm{e}, \mathrm{mu}$, em, ep, mum and mup.

\section{Photon cuts}

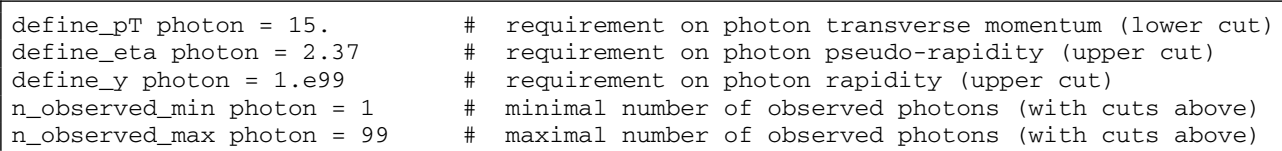

requirements defined here can be accessed in the definition of distributions, see Sect. 5.1.3. ${ }^{22}$

Analogous blocks can be processed by MATRIX for the particle groups bjet and $1 j e t$, which denote bottom jets and light jets (i.e. all jets, but the bottom jets), respectively. Note that a computation with bottom quarks treated as massless requires a jet involving a $b \bar{b}$ pair from a $g \rightarrow b \bar{b}$ splitting to be considered as a light jet, to guarantee observables to be IR safe. We thus define our $b$-jets by assigning bottomcharges to the partons: jets that contain a net bottom charge are considered bottom jets, whereas jets containing a $b \bar{b}$ pair are treated as light jets.

\footnotetext{
$\overline{21}$ Note that setting n_observed_min jet $=1$ would effectively reduce any (N)NLO calculation for the production of a final state $F$ in MATRIX to be only a $(\mathrm{N}) \mathrm{LO}$ accurate calculation for the production of $F+$ jet. On the other hand, setting n_observed_max jet $=0$ would impose a veto against events that contain any jets that fulfil the defined requirements on jets.

22 Accordingly, the defined particle group is also accessible within the $\mathrm{C}++$ code as discussed for the definition of new dynamic scales, cuts and observables for distributions by the advanced user in Appendix B.
}

Similarly, due to this block the photons in the particle group photon, which have passed the isolation criterion defined above, have a transverse momentum greater than $15 \mathrm{GeV}$ and absolute pseudo-rapidity smaller than 2.37 , and the presence of at least one such isolated photon is required. Note that for the cross section to be IR finite, the number of identified photons in the final state must be equal to the total number of photons in the final state of a process.

\begin{abstract}
${ }^{23}$ We stress again that any lepton in the particle group lep fulfils the defined (rapidity) requirements, irrespective of whether n_observed_min lep or n_observed_max lep require the presence of a minimal or maximal number of such leptons in the event. This is important to bear in mind when using lep to define distributions in Sect. 5.1.3. Even in a fully inclusive phase space without fiducial cuts, any distribution using lep will be affected by the defined (rapidity) requirements in the file parameter. dat on the leptons. Of course, the equivalent is true for any of the other particle groups.
\end{abstract}




\section{Heavy-boson cuts}

\section{Process-specific cuts}

A number of cuts are defined individually for each process. They enable a realistic definition of fiducial phase

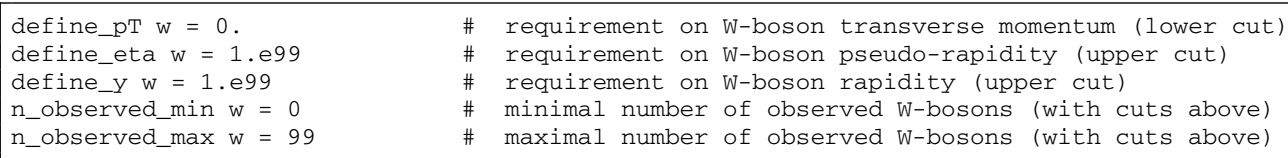

Equivalent blocks are available for the particle groups of heavy bosons, namely w, wm, wp, $\mathrm{z}$ and $\mathrm{h}$. The above example does not impose any requirements on $W$ bosons, as needed for a fully inclusive cross section.

\section{Neutrino cuts}

spaces as used in experimental measurements. For every process-specific cut there is usually one integer-valued switch (user_switch) to either turn on and off a certain cut or to choose between different options. Moreover, each switch typically comes with one or more realvalued parameters (user_cut) which are only active

define_pT missing $=30$.

\# requirement on $\mathrm{pT}$ of sum of all neutrinos (lower cut)

The particle group missing contains only the missing energy vector, given by the sum of all neutrino momenta. In processes with neutrinos this particle group can be used to impose a minimum requirement on the total missing transverse momentum in the event. The example above sets $p_{T}^{\text {miss }}>30 \mathrm{GeV}$.

In particular for technical checks it might be useful to access neutrinos also as individual particles. To do so, MATRIX can process blocks for the particle groups nua, nu, nux, nea, nma, ne, nex, nm and nmx. if the respective switch is turned on. There are a number of predefined process-specific cuts for each process, all of which are defined directly inside the $\mathrm{C}++$ code in the file MATRIX_v1.0.0/prc/\$ process_id\}/user/ specify.cuts.cxx; the list of predefined (processspecific) cuts for each process is documented in Sect. 5.2. A user interested in setting a specific cut which has not been implemented yet for a certain process is advised to contact the authors. ${ }^{24}$

For $Z \gamma$ production, e.g., the following predefined cuts are accessible in the file parameter. dat.

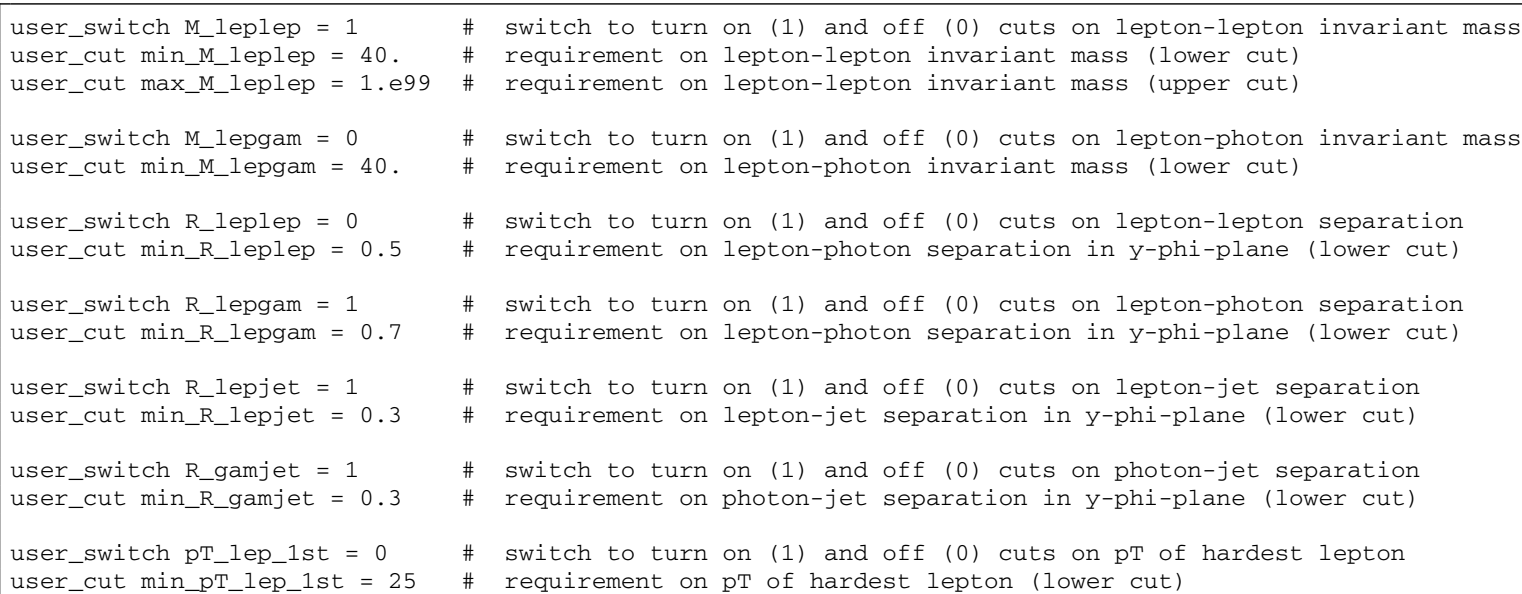

$\overline{24}$ A short description on how to add user-specified scales, cuts and distributions to the $\mathrm{C}++$ code is given in Appendix B for the advanced user. 


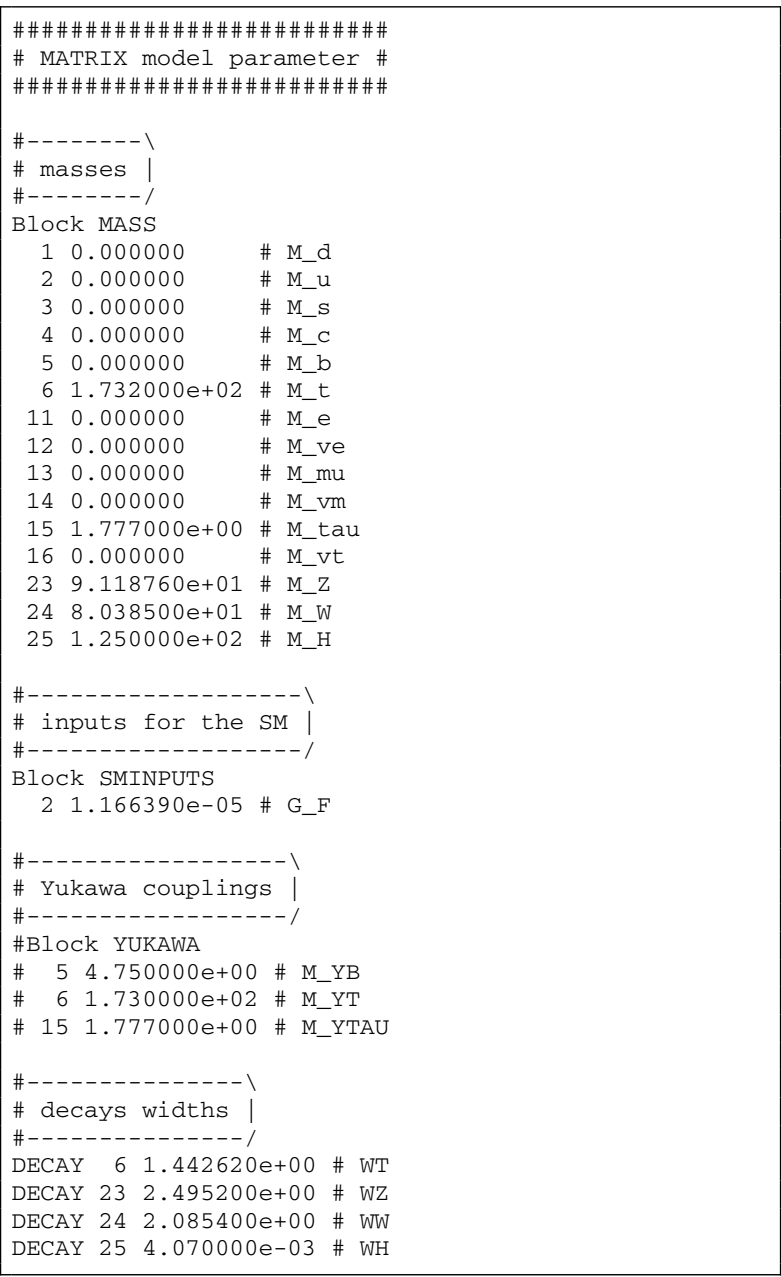

The Block Yukawa is currently not used, which is why it is commented.

In the first release of MATRIX, only on- and off-shell $W$ boson production allow for a non-trivial CKM matrix. This feature will be added also for other processes like $W \gamma$ and $W^{ \pm} Z$ production in a future update. The CKM parameters are controlled in the file model. dat of these processes through additional Blocks. The user may choose between three different setups. The default is a complete CKM matrix, where each of the entries may be set individually using Block CKM as defined below. top-related CKM entry has no effect on the processes considered in MATRIX.

A second option to use a non-trivial CKM matrix is through the Cabibbo angle $\theta_{c}$, by adding the Block VCKMIN as follows:

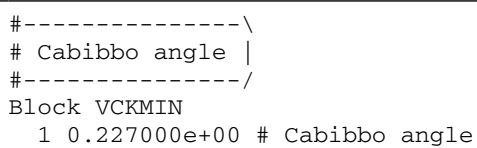

This enables mixing only between the first two generations, while turning off any mixing with the third generation, i.e. by setting internally $V_{u d}=\cos \left(\theta_{c}\right), V_{u s}=\sin \left(\theta_{c}\right)$, $V_{c d}=-\sin \left(\theta_{c}\right), V_{c s}=\cos \left(\theta_{c}\right), V_{t b}=1$, and $V_{u b}=V_{c b}=$ $V_{t d}=V_{t s}=0$. Note that only Block CKM or Block VKCMIN may be present in the file model. dat at the same time.

Finally, if both blocks are absent, a trivial CKM matrix (no mixing) is used.

\subsubsection{Settings in distribution. dat}

\subsubsection{General structure}

In the file distribution. dat the user can define histograms for distributions which are filled during the run. Each distribution is represented by one block containing the following parameters:

distributionname Unique user-defined label (string) of the distribution for identification at the end of the run; every distributionname starts a new block. Code will stop if the same distribution identifier is used twice.

distributiontype Type identifier (string) of the observable to be binned. MATRIX has a number of predefined observables, which are summarized in Table 4. A user interested in a specific distribution which has not been implemented yet is advised to contact the authors. ${ }^{25}$

particle $j$ Specification of particles entering the definition of the observable to be binned. Several final-states particles may be grouped into one particle. The general form is as follows:

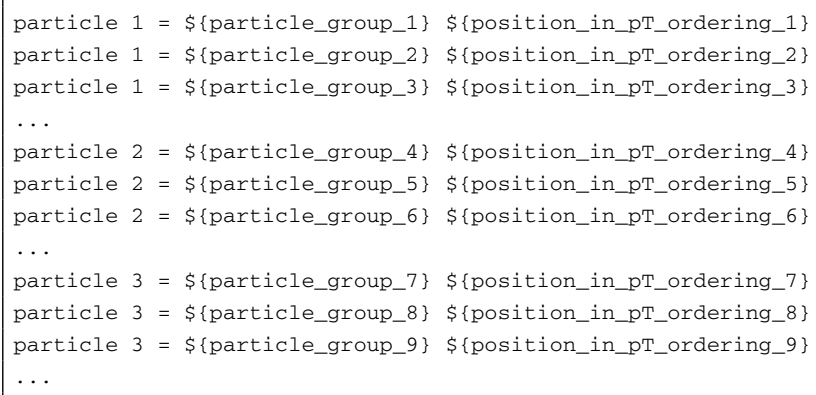

25 A short description on how to add user-specified scales, cuts and distributions to the $\mathrm{C}++$ code is given in Appendix $\mathrm{B}$ for the advanced user. 
Each $\$\{$ particle_group_i $\}$ is given by one of the particle groups defined in Table 3 , and $\$$ \{position_in_pT_ ordering_i\} is an integer which determines the desired position in the $p_{T}$-ordering of the respective group. For instance, lep 2 corresponds to the second-hardest lepton in the final state. If particle $j$ has several entries, the respective 4-momenta are summed to define the momentum of particle j. ${ }^{26}$ How many particles $(j=$ $1,2,3, \ldots)$ are allowed or required depends on the observable under consideration. Many observables use only one particle entry, i.e. only particle 1 , others that determine the distance or angle between two particles require two particles,i.e.particle 1 andparticle 2. Table 4 specifies this behaviour for each of the predefined observables.

binning_type Defines how the binning is performed. It may be set to linear, logarithmic or irregular (if not specified, linear is used as default):

- The setting linear requires the definition of three inputs out of startpoint, endpoint, binnumber and binwidth. The fourth one is uniquely defined then. Defining all four parameters results in a stop of the $\mathrm{C}++$ code if they are inconsistent.

- The setting logarithmic requires the definition of startpoint, endpoint and binnumber. The widths of the resulting bins are determined equidistantly on a logarithmic scale from this input.

- The setting irregular facilitates the definition of an arbitrary (not necessarily equidistant) binning, which is specified by the input parameter edges.

startpoint Left endpoint of the first bin (real number).

endpoint Right endpoint of the last bin (real number). binnumber Number of bins in the histogram (integer).

binwidth Width of each bin in the histogram (real number).

edges Edges (real numbers) of an irregular histogram, specified by $a_{0}: a_{1}: \cdots: a_{n}$ for $n$ bins.

\subsubsection{Examples}

We give a few examples on how proper distributions may be defined for the sample process of $Z \gamma$ production (examples

\footnotetext{
$\overline{26}$ This provides a simple way to access distributions of combined particles, such as a $Z$ boson determined by its two decay leptons. We note that combined (reconstructed) particles are defined for certain processes (see, e.g., Sect. 5.2.4.4) as additional particle groups via userdefined particles. This is particularly useful if the definition of such particle requires a certain pairing prescription, e.g. the reconstruction of a $Z$ boson in a same-flavour channel with more than two leptons. An advanced user may use this concept to define his own particle groups, see Appendix B.2.
}

can be found also in the file distribution. dat of each process).

- Transverse momentum of the hardest lepton, regularly binned in 200 bins from $0-1000 \mathrm{GeV}$ (i.e. in $5 \mathrm{GeV}$ steps):

$\begin{array}{ll}\text { distributionname } & =\mathrm{pT} \text { Tep1 } \\ \text { distributiontype } & =\mathrm{pT} \\ \text { particle } 1 & =1 \mathrm{pp} 1 \\ \text { startpoint } & =0 \\ \text { endpoint } & =1000 \\ \text { binnumber } & =200\end{array}$

- Transverse momentum of the second-hardest lepton, regularly binned from $0-1000 \mathrm{GeV}$ in $5 \mathrm{GeV}$ steps (i.e. in 200 bins):

$\begin{array}{ll}\text { distributionname } & =\mathrm{pT} \text { Tep2 } \\ \text { distributiontype } & \mathrm{pT} \\ \text { particle } 1 & =1 \mathrm{ep} 2 \\ \text { startpoint } & =0 . \\ \text { endpoint } & =1000 \\ \text { binwidth } & =5 .\end{array}$

- Transverse momentum of the hardest photon with irregular edges (as used by ATLAS in the $7 \mathrm{TeV}$ analysis for Z $\gamma$ [106]):

$\begin{array}{|ll|}\text { distributionname } & =\mathrm{pT} \text { Tgamma_ATLAS } \\ \text { distributiontype } & =\mathrm{pT} \\ \text { particle } 1 & =\text { photon } 1 \\ \text { binningtype } & =\text { irregular } \\ \text { edges } & =0 .: 15 .: 20 .: 30 .: 40 .: 60 .: 100 .: 1000 .: 3500\end{array}$

- Invariant mass of the pair formed by the hardest and the second-hardest lepton, binned from $0-1000 \mathrm{GeV}$ in $10 \mathrm{GeV}$ steps:

\begin{tabular}{|ll} 
distributionname & $=$ m_lep1_lep2 \\
distributiontype & $=\mathrm{m}$ \\
particle 1 & $=1 \mathrm{ep} 1$ \\
particle 1 & $=1 \mathrm{ep} 2$ \\
startpoint & $=0$. \\
endpoint & $=1000$. \\
binwidth & $=10$.
\end{tabular}

- Distance in $y-\phi$ plane between the hardest electron and the hardest positron, binned from $0-10$ in 0.1 steps:

$\begin{array}{ll}\text { distributionname } & =\mathrm{dR} \text { em1_ep1 } \\ \text { distributiontype } & =\mathrm{dR} \\ \text { particle 1 } & =\mathrm{em} 1 \\ \text { particle 2 } & =\mathrm{ep} 1 \\ \text { startpoint } & =0 . \\ \text { endpoint } & =10 . \\ \text { binwidth } & =0.1\end{array}$

The default file distribution. dat contains further examples and information, as well as instructions on how to define distributions in this format.

\subsection{Process-specific settings}

In this section we provide information specific to the individual processes. Below we list all processes available in MATRIX by their respective $\$\{$ process_id\}, summarize 
Table 4 Predefined distributions available in MATRIX. These distributions can be used in a distribution block of the file distribution.dat and require to specify the parameter(s) particle $j, j=1, \ldots, m(m \geq 1)$. Some observables behave differently for a different number of defined particles $m$ : In these cases

\begin{tabular}{|c|c|c|}
\hline Identifier & Binned variable & Description \\
\hline $\mathrm{pT}$ & $\sum_{j=1}^{m} p_{T}^{j}$ & Scalar sum of transverse momenta of particle 1 to particle $\mathrm{m}$ \\
\hline $\mathrm{m}$ & $m\left(p^{1}\right)$ & Invariant mass of particle 1 \\
\hline $\mathrm{dm}$ & $m\left(p^{1}\right)-m\left(p^{2}\right)$ & Invariant-mass difference between particle 1 and particle 2 \\
\hline $\mathrm{absdm}$ & $\left|m\left(p^{1}\right)-m\left(p^{2}\right)\right|$ & $\begin{array}{l}\text { Absolute invariant-mass difference between particle } 1 \text { and } \\
\text { particle } 2\end{array}$ \\
\hline $\operatorname{mmin}$ & $\min \left(m\left(p^{1}\right), m\left(p^{2}\right)\right)$ & Minimal invariant-mass of particle 1 and particle 2 \\
\hline $\operatorname{mmax}$ & $\max \left(m\left(p^{1}\right), m\left(p^{2}\right)\right)$ & Maximal invariant-mass of particle 1 and particle 2 \\
\hline $\mathrm{Y}$ & $y\left(p^{1}\right)$ & Rapidity of particle 1 \\
\hline absy & $\left|y\left(p^{1}\right)\right|$ & Absolute rapidity of particle 1 \\
\hline dy & $y\left(p^{1}\right)-y\left(p^{2}\right)$ & Rapidity difference between particle 1 and particle 2 \\
\hline absdy & $\left|y\left(p^{1}\right)-y\left(p^{2}\right)\right|$ & $\begin{array}{l}\text { Absolute rapidity difference between particle } 1 \text { and } \\
\text { particle } 2\end{array}$ \\
\hline dabsy & $\left|y\left(p^{1}\right)\right|-\left|y\left(p^{2}\right)\right|$ & $\begin{array}{l}\text { Difference between absolute rapidities of particle } 1 \text { and } \\
\text { particle } 2\end{array}$ \\
\hline absdabsy & ||$y\left(p^{1}\right)|-| y\left(p^{2}\right)||$ & $\begin{array}{l}\text { Absolute difference between absolute rapidities of particle } 1 \text { and } \\
\text { particle } 2\end{array}$ \\
\hline eta & $\eta\left(p^{1}\right)$ & Pseudo-rapidity of particle 1 \\
\hline abseta & $\left|\eta\left(p^{1}\right)\right|$ & Absolute pseudo-rapidity of particle 1 \\
\hline deta & $\eta\left(p^{1}\right)-\eta\left(p^{2}\right)$ & Pseudo-rapidity difference between particle 1 and particle 2 \\
\hline absdeta & $\left|\eta\left(p^{1}\right)-\eta\left(p^{2}\right)\right|$ & $\begin{array}{l}\text { Absolute pseudo-rapidity difference between particle } 1 \text { and } \\
\text { particle } 2\end{array}$ \\
\hline dabseta & $\left|\eta\left(p^{1}\right)\right|-\left|\eta\left(p^{2}\right)\right|$ & $\begin{array}{l}\text { Difference between absolute pseudo-rapidities of particle } 1 \text { and } \\
\text { particle } 2\end{array}$ \\
\hline absdabseta & ||$\eta\left(p^{1}\right)|-| \eta\left(p^{2}\right)||$ & $\begin{array}{l}\text { Absolute difference between absolute pseudo-rapidities of } \\
\text { particle } 1 \text { and particle } 2\end{array}$ \\
\hline phi & $\phi\left(p^{1}\right)$ & Azimuthal angle of particle 1 \\
\hline phi & $\Delta \phi\left(p^{1}, p^{2}\right)$ & $\begin{array}{l}\text { Difference in azimuthal angle between particle } 1 \text { and } \\
\text { particle } 2\end{array}$ \\
\hline$d R$ & $\sqrt{\left[\Delta y\left(p^{1}, p^{2}\right)\right]^{2}+\left[\Delta \phi\left(p^{1}, p^{2}\right)\right]^{2}}$ & Distance in $y$ - $\phi$-plane between particle 1 and particle 2 \\
\hline dReta & $\sqrt{\left[\Delta \eta\left(p^{1}, p^{2}\right)\right]^{2}+\left[\Delta \phi\left(p^{1}, p^{2}\right)\right]^{2}}$ & Distance in $\eta$ - $\phi$-plane between particle 1 and particle 2 \\
\hline ET & $\sum_{j=1}^{m} E_{T}\left(p^{j}\right) \equiv \sum_{j=1}^{m} \sqrt{\left[m\left(p^{j}\right)\right]^{2}+\left[p_{T}^{j}\right]^{2}}$ & Scalar sum of transverse masses of particle 1 to particle m \\
\hline $\mathrm{mT}$ & $E_{T}\left(p^{1}\right)$ & Transverse mass of particle 1 \\
\hline $\mathrm{mT}$ & $\sqrt{\left[\sum_{j=1}^{m} E_{T}\left(p^{j}\right)\right]^{2}-\left[p_{T}\left(\sum_{j=1}^{m} p^{j}\right)\right]^{2}}$ & $\begin{array}{l}\text { Transverse mass, defined with all neutrinos in particle } 1 \text { and all } \\
\text { other particles in particle } 2 \text { to particle m }\end{array}$ \\
\hline pTveto & $\sigma\left(p_{T}^{1}<p_{T, \text { veto }}\right)$ & $\begin{array}{l}\text { Cumulative cross section with a veto on } p_{T} \text { of particle } 1 \text { as a } \\
\text { function of } p_{T, \text { veto }}\end{array}$ \\
\hline multiplicity & $N$ & Distribution in number of identified objects of type particle 1 \\
\hline muR & $\mu_{R}$ & Distribution in renormalization scale (no particle $j$ definition) \\
\hline $\mathrm{muF}$ & $\mu_{F}$ & Distribution in factorization scale (no particle $j$ definition) \\
\hline
\end{tabular}

the predefined process-specific cuts and dynamic scales, and, where applicable, we give additional process-specific information. the respective options are given in separate rows. We use the shorthand notation $p^{j}$ for the momentum of particle $j$. Note that, if particle $j$ has several entries of particles, $p^{j}$ is the sum of their momenta, which provides a straightforward way to access trivially reconstructed particles (e.g. a single $Z$ boson from its decay leptons)

Invariant mass of particle 1

particle 2

bsolute invariant-mass difference between particle 1 and

-mass of particle 1 and particle 2

Rapidity of particle 1

Absolute rapidity of particle 1

Rapidity difference between particle 1 and particle 2

Absolute rapidity difference between particle 1 and

Difference between absolute rapidities of particle 1 and

particle

Pseudo-rapidity of particle 1

Absolute pseudo-rapidity of particle

Pseudo-rapidity difference between particle 1 and particle 2

Absolute pseudo-rapidity difference between particle 1 and

Detween absolute pseudo-rapidities of particle 1 and

particle 1 and particle 2

Difference in azimuthal angle between particle 1 and

Distance in $\eta$ - $\phi$-plane between particle 1 and particle 2

Scalar sum of transverse masses of particle 1 to particle m

Transverse mass, defined with all neutrinos in particle 1 and all

function of $p_{T \text {,veto }}$

Distribution in number of identified objects of type particle 1

Distribution in renormalization scale (no particle $j$ definition) Deale (no particle j definition)

In addition to the standard cuts on particle groups, discussed in Sect. 5.1.1.4, process-specific fiducial cuts are predefined via an integer-valued parameter user_switch in 
combination with none, one or more real-valued parameters user_cut. For a user_switch XXX together with corresponding user_cut XXX_A, user_cut XXX_B, and so forth, we adopt the notation

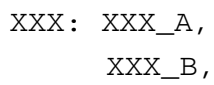

to list all available predefined cuts in the respective file parameter. dat of each process. A detailed explanation for each of these cuts is given in Appendix A. ${ }^{27}$

As outlined in Sect. 5.1.1.2, dynamic scales are set by the switch dynamic_scale in the file parameter.dat, and there are two default scales for all processes: the invariant and the transverse mass of the colourless system. Any additional predefined scale implemented for a process is stated below, and the adopted nomenclature is summarized in Table 5.

We note that all leptons are considered massless throughout all computations. This implies that, e.g., electrons may be considered as muons and vice versa in order to get results for other lepton flavours. Thus, a process like $p p / p \bar{p} \rightarrow e^{-} e^{+}$ is fully equivalent to $p p / p \bar{p} \rightarrow \mu^{-} \mu^{+}$, and only the former is provided in MATRIX. The same holds for more involved processes such as $p p / p \bar{p} \rightarrow e^{-} \mu^{-} e^{+} \bar{v}_{\mu}$ and $p p / p \bar{p} \rightarrow$ $\mu^{-} e^{-} \mu^{+} \bar{v}_{e}$ if the cuts do not depend on the lepton flavour. Since we provide only the $p p / p \bar{p} \rightarrow e^{-} \mu^{-} e^{+} \bar{v}_{\mu}$ channel, $p p / p \bar{p} \rightarrow \mu^{-} e^{-} \mu^{+} \bar{v}_{e}$ for different muon and electron cuts can be simply computed by using $p p / p \bar{p} \rightarrow e^{-} \mu^{-} e^{+} \bar{v}_{\mu}$ with muon cuts implemented for electrons and vice versa.

An alternative which will be supported in a future release is an exchange of electrons and muons by means of the parameter process_class. For every process where this is relevant, a separate file parameter. dat will be provided inside its folder input, which can be used instead of the original file parameter. dat of the process to run with exchanged electrons and muons. For example, for different-flavour $W^{ \pm} Z$ production $(\$\{$ process_id $\}=$ ppemexnmx 04) an additional file with process_class $=$ ppmemxnex04 instead of process_class = ppemexnmx04 will be used to calculate the process $p p / p \bar{p} \rightarrow \mu^{-} e^{-} \mu^{+} \bar{v}_{e}$ instead of $p p / p \bar{p} \rightarrow e^{-} \mu^{-} e^{+} \bar{v}_{\mu}$, and all scales, cuts, distributions, etc. are to be formulated directly for the actual particles of this new process.

All processes available in MATRIX are discussed in the following, grouped into Higgs boson production (Sect. 5.2.1), vector-boson production (Sect. 5.2.2), diphoton and vectorboson plus photon production (Sect. 5.2.3), and vector-boson pair production (Sect. 5.2.4). The process-specific cuts are explained in detail in Appendix A.

\footnotetext{
27 The links embedded in the arXiv version of this paper for each cut in this section can be used to jump to the corresponding explanation in Appendix A, if supported by the PDF viewer in use.
}

Table 5 Symbols used in the definition of dynamic scales throughout this section. The transverse mass of a particle, or a sum of particle momenta, $X$ is defined as $m_{T, X}=\sqrt{m_{X}^{2}+p_{T, X}^{2}}$, with $p_{T, X}$ and $m_{X}^{2}$ being its transverse momentum and invariant mass, respectively

\begin{tabular}{|c|c|}
\hline$m_{Z}:$ & Mass of the $Z$ boson \\
\hline$m_{W}:$ & Mass of the $W$ boson \\
\hline$p_{T, e^{-} e^{+}}:$ & $\begin{array}{l}\text { Transverse momentum of the reconstructed } Z \\
\text { boson (electron pair) }\end{array}$ \\
\hline$p_{T, \mu^{-} \mu^{+}}:$ & $\begin{array}{l}\text { Transverse momentum of the reconstructed } Z \\
\text { boson (muon pair) }\end{array}$ \\
\hline$p_{T, v_{\mu} \bar{v}_{\mu}}:$ & $\begin{array}{l}\text { Transverse momentum of the reconstructed } Z \\
\text { boson (neutrino pair) }\end{array}$ \\
\hline$p_{T, Z_{\mathrm{rec}}}:$ & $\begin{array}{l}\text { Transverse momentum of the reconstructed } Z \\
\text { boson (see main text) }\end{array}$ \\
\hline$p_{T, Z_{i, \mathrm{rec}}}:$ & $\begin{array}{l}\text { Transverse momentum of the respective } \\
\text { reconstructed } Z \text { boson (see main text) }\end{array}$ \\
\hline$p_{T, e^{ \pm} v_{e}}:$ & $\begin{array}{l}\text { Transverse momentum of the reconstructed } W \\
\text { boson (electron-neutrino pair) }\end{array}$ \\
\hline$p_{T, \mu^{ \pm}} v_{\mu}:$ & $\begin{array}{l}\text { Transverse momentum of the reconstructed } W \\
\text { boson (muon-neutrino pair) }\end{array}$ \\
\hline$p_{T, W_{\mathrm{rec}}^{ \pm}}:$ & $\begin{array}{l}\text { Transverse momentum of the reconstructed } W \\
\text { boson (see main text) }\end{array}$ \\
\hline$m_{T, e^{-} e^{+}}:$ & $\begin{array}{l}\text { Transverse mass of the reconstructed } Z \text { boson } \\
\text { (electron pair) }\end{array}$ \\
\hline$m_{T, \mu^{-} \mu^{+}}:$ & $\begin{array}{l}\text { Transverse mass of the reconstructed } Z \text { boson } \\
\text { (muon pair) }\end{array}$ \\
\hline$m_{T, v_{e} \bar{v}_{e}}$ & $\begin{array}{l}\text { Transverse mass of the reconstructed } Z \text { boson } \\
\text { (neutrino pair) }\end{array}$ \\
\hline$m_{Z_{\mathrm{rec}}}:$ & $\begin{array}{l}\text { Transverse mass of the reconstructed } Z \text { boson } \\
\text { (see main text) }\end{array}$ \\
\hline$m_{T, Z_{i, \mathrm{rec}}}:$ & $\begin{array}{l}\text { Transverse mass of the respective reconstructed } \\
Z \text { boson (see main text) }\end{array}$ \\
\hline$m_{T, e^{ \pm} v_{e}}:$ & $\begin{array}{l}\text { Transverse mass of the reconstructed } W \text { boson } \\
\text { (electron-neutrino pair) }\end{array}$ \\
\hline$m_{T, \mu^{ \pm}} v_{\mu}:$ & $\begin{array}{l}\text { Transverse mass of the reconstructed } W \text { boson } \\
\text { (muon-neutrino pair) }\end{array}$ \\
\hline$m_{T, W_{\mathrm{rec}}^{ \pm}}:$ & $\begin{array}{l}\text { Transverse mass of the reconstructed } W \text { boson } \\
\text { (see main text) }\end{array}$ \\
\hline$m_{T, \gamma}\left(p_{T, \gamma}\right):$ & Transverse mass (momentum) of the photon \\
\hline
\end{tabular}

\subsubsection{Higgs boson production}

\subsubsection{1 pph21 $(p p / p \bar{p} \rightarrow H)$}

On-shell Higgs boson production has no process-specific cuts or dynamic scales. The process is computed in the infinitetop-mass approximation by using an effective field theory where the top quark is integrated out.

\subsubsection{Vector-boson production}

This group contains both the on-shell and the off-shell production of a single vector boson. Whereas the former processes feature cuts and distributions only with respect to the 
on-shell final state, the off-shell processes give access to, in principle, arbitrary phase-space selection cuts and distributions of the leptons. The phenomenologically irrelevant process of $p p / p \bar{p} \rightarrow v_{e} \bar{v}_{e}$ production has been added as it might be useful for technical checks.

\subsubsection{1 ppz01 ( $p p / p \bar{p} \rightarrow Z)$}

On-shell $Z$-boson production has no process-specific cuts or dynamic scales.

$$
\begin{aligned}
& \text { 5.2.2.2 } \operatorname{ppw01}\left(p p / p \bar{p} \rightarrow W^{-}\right), \\
& \operatorname{ppwx01}\left(p p / p \bar{p} \rightarrow W^{+}\right)
\end{aligned}
$$

On-shell $W^{ \pm}$-boson production has no process-specific cuts or dynamic scales. The process includes a non-trivial CKM matrix, which the user may modify, see Sect. 5.1.2.

\subsubsection{3 ppeex02 $\left(p p / p \bar{p} \rightarrow e^{-} e^{+}\right)$}

Off-shell $Z$-boson production ${ }^{28}$ with decay to leptons includes the following predefined cuts:

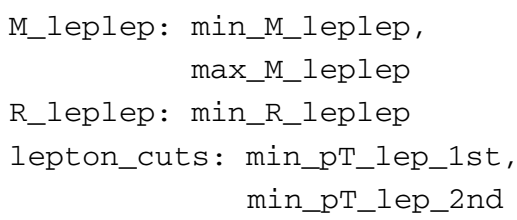

No process-specific dynamic scales are implemented.

If cuts are applied, this process may feature a peculiarly strong dependence on the value of $r_{\text {cut }}$ in the $q_{T^{-}}$ subtraction procedure, see Sect. 7. The $p p / p \bar{p} \rightarrow e^{-} e^{+}$process therefore features a switch switch_qT_accuracy in the file parameter.dat, which allows the user to decrease the uncertainty induced by the $q_{T}$-subtraction procedure at NNLO, at the cost of a slower numerical convergence:

switch_qT_accuracy $=0$ Uses the default value $r_{\text {cut }}=0.15 \%$ with fast numerical convergence.

switch_qT_accuracy $=1$ Uses $r_{\text {cut }}=0.05 \%$ with reduced uncertainty, but longer runtime.

We recommend to use switch_qT_accuracy $=0$ if the targeted precision of the extrapolated cross-section prediction $\left(r_{\mathrm{cut}} \rightarrow 0\right)$ is of the order of $0.5 \%-1 \%$. To achieve results with numerical precision of $0.1 \%-0.5 \%$, switch_qT_accuracy $=1$ should be used.

\subsubsection{4 ppnenex02 $\left(p p / p \bar{p} \rightarrow v_{e} \bar{v}_{e}\right)$}

Off-shell $Z$-boson production with decay to neutrinos has no process-specific cuts or dynamic scales.

$\overline{{ }^{28} \text { Note that this }}$ process includes also off-shell photon contributions.

\subsubsection{5 ppenex02 $\left(p p / p \bar{p} \rightarrow e^{-} \bar{v}_{e}\right)$, ppexne02 $\left(p p / p \bar{p} \rightarrow e^{+} v_{e}\right)$}

Off-shell $W^{ \pm}$-boson production has no process-specific cuts or dynamic scales. The process includes a non-trivial CKM matrix, which the user may modify, see Sect. 5.1.2.

\subsubsection{Diphoton and vector-boson plus photon production}

This group contains both the diphoton process and the $V \gamma$ processes with off-shell leptonic decays of the heavy vector bosons $V$.

All processes with isolated photons in the final state have a peculiarly strong dependence on the value of $r_{\text {cut }}$ in the $q_{T}$-subtraction procedure, see Sect. 7. For this reason the estimated uncertainty induced by finite $r_{\text {cut }}$ values is particularly large in these processes. The photon processes therefore feature a switch switch_qT_accuracy in the file parameter. dat, which allows the user to decrease the uncertainty induced by the $q_{T}$-subtraction procedure at NNLO, at the cost of a slower numerical convergence: switch_qT_accuracy $=0$ Uses the default value $r_{\text {cut }}=0.15 \%$ with fast numerical convergence.

switch_qT_accuracy $=1$ Uses $r_{\text {cut }}=0.05 \%$ with reduced uncertainty, but longer runtime.

We recommend to use switch_qT_accuracy $=0$ if the targeted precision of the extrapolated cross-section prediction $\left(r_{\text {cut }} \rightarrow 0\right)$ is of the order of $0.5 \%-1 \%$. To achieve results with numerical precision of $0.1 \%-0.5 \%$, switch_qT_accuracy $=1$ should be used.

\subsubsection{1 ppaa02 $(p p / p \bar{p} \rightarrow \gamma \gamma)$}

Diphoton production includes the following predefined cuts:

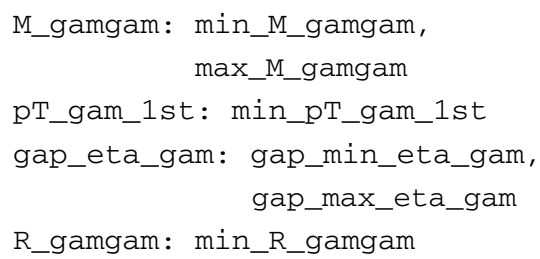

No process-specific dynamic scales are implemented.

\subsubsection{2 ppeexa03 $\left(p p / p \bar{p} \rightarrow e^{-} e^{+} \gamma\right)$}

$Z \gamma$ production $[30,31]$ with $Z$-boson decay to charged leptons $^{29}$ includes the following predefined cuts:

\footnotetext{
${ }^{29}$ Note that this process includes also $\gamma^{*} \gamma$ contributions, where one photon is off-shell and decays to leptons, and $Z / \gamma^{*}$ production with a subsequent decay $Z / \gamma^{*} \rightarrow e^{-} e^{+} \gamma$.
} 
M_leplep: min_M_leplep

M_lepgam: min_M_lepgam

R_leplep: min_R_leplep

R_lepgam: min_R_lepgam

R_lepjet: min_R_lepjet

R_gamjet: min_R_gamjet

pT_lep_1st: min_pT_lep_1st

The process facilitates the following additional predefined dynamic scales (symbols in Table 5):

\begin{tabular}{ll}
\hline dynamic_scale $=3:$ & $\mu=m_{T, \gamma} \equiv p_{T, \gamma}$ \\
dynamic_scale $=4:$ & $\mu=m_{T, e^{-} e^{+}}$ \\
dynamic_scale $=5:$ & $\mu=\sqrt{m_{T, \gamma} \cdot m_{T, e^{-}} e^{+}}$ \\
dynamic_scale $=6:$ & $\mu=\sqrt{m_{Z}^{2}+m_{T, \gamma}^{2}}$ \\
\hline
\end{tabular}

\subsubsection{3 ppnenexa03 $\left(p p / p \bar{p} \rightarrow v_{e} \bar{\nu}_{e} \gamma\right)$}

$Z \gamma$ production [31] with $Z$-boson decay to neutrinos includes the following predefined cuts:

R_gamjet: min_R_gamjet

The process facilitates the following additional predefined dynamic scales (symbols in Table 5): dynamic_scale $=3$ :

dynamic_scale $=4$ :

dynamic_scale $=5$ :

dynamic_scale $=6$ :
$\mu=m_{T, \gamma} \equiv p_{T, \gamma}$

$\mu=m_{T, v_{e}} \bar{v}_{e}$

$\mu=\sqrt{m_{T, \gamma} \cdot m_{T, v_{e}} \bar{v}_{e}}$

$\mu=\sqrt{m_{Z}^{2}+m_{T, \gamma}^{2}}$

\subsubsection{4 ppenexa03 $\left(p p / p \bar{p} \rightarrow e^{-} \bar{v}_{e} \gamma\right)$, ppexnea03 $\left(p p / p \bar{p} \rightarrow e^{+} v_{e} \gamma\right)$}

$W^{ \pm} \gamma$ production [31] with leptonic $W$-boson decay ${ }^{30}$ includes the following predefined cuts:

R_lepgam: min_R_lepgam

R_lepjet: min_R_lepjet

R_gamjet: min_R_gamjet

mT_CMS: min_mT_CMS

gap_eta_gam: gap_min_eta_gam,

$$
\text { gap_max_eta_gam }
$$

The process facilitates the following additional predefined dynamic scales (symbols in Table 5):

\footnotetext{
$\overline{30}$ Note that this process includes also contributions from $W^{ \pm}$production with a subsequent decay $W^{+} \rightarrow e^{+} v_{e} \gamma$ or $W^{-} \rightarrow e^{-} \bar{v}_{e} \gamma$, respectively.
}

dynamic_scale $=3$ :

dynamic_scale $=4$ :

dynamic_scale $=5$ :

dynamic_scale $=6$ :

\subsubsection{Vector-boson pair production}

This group contains both the on-shell and the off-shell production of a vector-boson pair. The on-shell production of a $W^{+} W^{-}$or a $Z Z$ pair allows selection cuts to be applied only on the vector bosons, and distributions in the vector-boson kinematics can be studied. The off-shell processes, on the other hand, give access to the full leptonic final states, i.e. they allow in principle arbitrary IR safe selection cuts on the leptons to be applied, and distributions in the kinematics of these leptons can be computed. Off-shell vector-boson pair production includes processes with different-flavour (DF) and same-flavour (SF) leptons in the final state. For the processes with two neutrinos and two leptons in the final state, the separation of $\mathrm{DF}\left(e^{-} e^{+} v_{\mu} \bar{v}_{\mu}\right)$ and $\mathrm{SF}\left(e^{-} e^{+} v_{e} \bar{v}_{e}\right)$ channels is done according to the underlying calculation, not to the experimental signature: For any analysis of two leptons plus missing transverse energy the predictions must be obtained by (incoherently) combining the DF and SF processes, i.e.

$$
\begin{aligned}
\sigma\left(e^{-} e^{+}+p_{\mathrm{T}, \mathrm{miss}}\right)= & \sigma\left(e^{-} e^{+} v_{e} \bar{v}_{e}\right)+\sigma\left(e^{-} e^{+} v_{\mu} \bar{v}_{\mu}\right) \\
& +\sigma\left(e^{-} e^{+} v_{\tau} \bar{v}_{\tau}\right) \\
= & \sigma\left(e^{-} e^{+} v_{e} \bar{v}_{e}\right)+2 \times \sigma\left(e^{-} e^{+} v_{\mu} \bar{v}_{\mu}\right)
\end{aligned}
$$

\section{Flavour-scheme choice and top-quark contamination in $W W$ production}

All processes including a pair of on- or off-shell $W$ bosons are subject to a contamination by off-shell top-quark contributions with $t \rightarrow W b$ decays. Such contributions enter radiative corrections in both the four-flavour scheme (4FS), where bottom quarks are treated as massive, and the fiveflavour scheme (5FS), where the bottom-quark mass is set to zero as all other light-quark masses. In case of $W^{+} W^{-}$ production, the 4FS has the advantage that the bottom quark appears only in the final state, and that the bottom-quark mass renders all partonic subprocesses with bottom quarks in the final state separately finite. Thus, the top-quark contamination is easily avoided by omitting bottom-quark emission subprocesses in this scheme, which are considered part of the (off-shell) top-pair background. Consequently, we use this 4FS approach as the default for any process that features an on- or off-shell $W^{+} W^{-}$pair, namely by setting flavour_scheme $=0$ in the file parameter. dat in combination with $m_{\mathrm{b}} \neq 0$ in the file model. dat. We note 
that this approach requires the use of consistent PDF sets with $n_{f}=4$ light parton flavours.

Alternatively, one can use the 5FS by setting flavour_scheme $=1, m_{\mathrm{b}}=0$ and choosing $n_{f}=5$ PDF sets. In this case, however, the top-quark contamination is not removed from the results. A numerical procedure to achieve a definition of the $W^{+} W^{-}$cross section without top-quark contamination in the 5FS, which has been used in Refs. [36,37], requires the repeated computation of the cross section for varying top-quark widths in order to approach the limit $\Gamma_{t} \rightarrow 0$ and thereby to isolate the contributions from single-top and top-pair production. As it has been shown in these references, the resulting top-subtracted $W^{+} W^{-}$cross sections calculated in the 4FS and the 5FS prescription, respectively, agree within 1-2\%, both at the inclusive level and with different sets of fiducial cuts applied. This justifies the use of the simpler 4FS computation for such processes.

\section{Off-shell $Z$ bosons in $Z Z$ production}

For off-shell $Z Z$-production processes the cuts may be arranged in a way that at least one of the $Z$ bosons is forced to be far in the off-shell region. For such cases these processes include an additional switch switch_off_shell in the file parameter. dat to improve the convergence of the computation in this phase-space region. This is relevant, e.g., when studying the $Z Z$ background in Higgs boson measurements. The default choice switch_off_shel1 =0 uses the standard setup for the grid generation (grid-run, see Sect. 4.4.1), which is suitable if both $Z$ bosons can simultaneously become resonant. Using switch_off_shell = 1 adapts the settings of the grid-run for cases where at least one $Z$ boson is off-shell.

\subsubsection{1 ppzz02 $(p p / p \bar{p} \rightarrow Z Z)$}

On-shell $Z Z$ production [33] has no process-specific cuts or dynamic scales.

\subsubsection{2 ppwxw02 $\left(p p / p \bar{p} \rightarrow W^{+} W^{-}\right)$}

On-shell $W^{+} W^{-}$production [36,37] has no process-specific cuts or dynamic scales.

By default (flavour_scheme $=0$ ) this process is computed in the 4FS, dropping all diagrams with final-state bottom quarks to remove the top-quark contamination. The 5FS can be chosen by setting flavour_scheme $=1$, where, however, the top-quark contamination is not removed from the results, since the recommended procedure is much more involved and requires several runs for the 5FS, see introduction of Sect. 5.2.4 for further details.

\subsubsection{3 ppemexmx04 (pp/p $\left.\bar{p} \rightarrow e^{-} \mu^{-} e^{+} \mu^{+}\right)$}

Off-shell $Z Z$ production [34] with $Z$-boson decays to DF leptons ${ }^{31}$ includes the following predefined cuts:

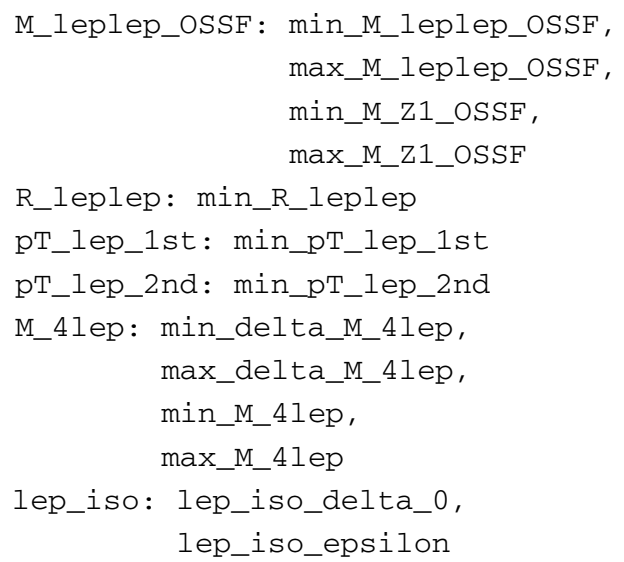

The process facilitates the following additional predefined dynamic scales (symbols in Table 5):

dynamic_scale $=3: \mu=\sqrt{m_{T, e^{-} e^{+}} \cdot m_{T, \mu^{-}} \mu^{+}}$

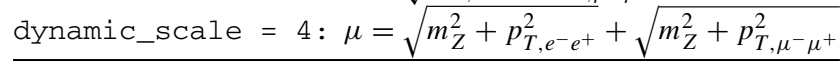

This process provides an additional switch switch_off_shell to speed up the grid-generation phase when considering at least one of the $Z$ bosons to be far off-shell; it should not be used otherwise, see introduction of Sect. 5.2.4 for further details.

\subsubsection{4 ppeeexex04 $\left(p p / p \bar{p} \rightarrow e^{-} e^{-} e^{+} e^{+}\right)$}

Off-shell $Z Z$ production [34] with $Z$-boson decays to SF leptons ${ }^{32}$ includes the following predefined cuts:

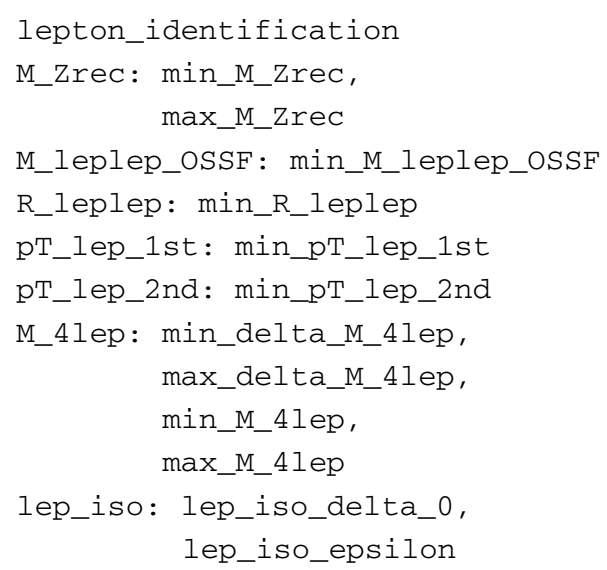

\footnotetext{
$\overline{31}$ Note that this process includes also $Z \gamma^{*}$ and $\gamma^{*} \gamma^{*}$ contributions with off-shell photons decaying to leptons, as well as $Z / \gamma^{*}$ production with a subsequent decay $Z / \gamma^{*} \rightarrow e^{-} \mu^{-} e^{+} \mu^{+}$.

${ }^{32}$ Note that this process includes also $Z \gamma^{*}$ and $\gamma^{*} \gamma^{*}$ contributions with off-shell photons decaying to leptons, as well as $Z / \gamma^{*}$ production with a subsequent decay $Z / \gamma^{*} \rightarrow e^{-} e^{-} e^{+} e^{+}$.
} 
Since this process features four SF leptons, two of which are positively and two negatively charged, the leptons cannot be unambiguously associated with the two parent $Z$ bosons as in the DF case. However, the experimental analyses often rely on cuts specific to (reconstructed) $Z$ bosons. Hence, in the SF channel an identification procedure is required to assign one opposite-charge same-flavour (OSSF) lepton pair to each of the $Z$ bosons. The parameter lepton_identification switches between such identification procedures of the $Z$ bosons as used by ATLAS and CMS. In both cases seven new particle groups are defined (see Sect. 5.1.1.4 and the related Table 3 for the standard particle groups), which makes them available in the definition of cuts, scales and distributions: Particle group Z1rec contains the $Z$ boson reconstructed from the OSSF lepton pair with its invariant mass closer to the $Z$-boson mass, labelled as $Z_{1 \text {,rec }}$, and particle group $Z 2$ rec contains the remaining OSSF lepton pair, labelled as $Z_{2, \text { rec }}$. Particle group Zrec is filled with both reconstructed $Z$ bosons in the standard $p_{T}$-ordering. The particle groups $1 \mathrm{mZ1}$, ImZ2, $1 \mathrm{pz} 1$ and $1 \mathrm{pz} 2$ contain the negatively and positively charged leptons that belong to the corresponding reconstructed $Z$ bosons, respectively, i.e. each of these groups has by definition only a single entry. Examples of the usage of these particle groups can be found in the file distribution. dat of this process. Furthermore, the predefined cut M_Zrec: min_M_Zrec, max_M_Zrec uses the respective particle groups identified corresponding to the setting of the switch lepton_identification. ${ }^{33}$

If lepton_identification $=0$ is set, the respective particle groups are not filled and thus cannot be used to define distributions. Also cuts and dynamic scales depending on the identification must not be used in this case, such as M_Zrec: min_M_Zrec, max_M_Zrec. In the following we outline the predefined pairing prescriptions.

The ATLAS pairing (lepton_identification = 1) considers all possible (two, in the theoretical computation) combinations to associate two OSSF lepton pairs with $Z_{1}=$ $e^{-} e^{+}$and $Z_{2}=e^{-1} e^{+t}$. The criterion to decide on the pairing is the sum of the absolute differences of their invariant masses to the $Z$-boson mass, i.e. $\left|m_{e^{-} e^{+}}-m_{Z}\right|+\left|m_{e^{-\prime} e^{+\prime}}-m_{Z}\right|$, and the assignment that minimizes this sum is associated with the reconstructed $Z$ bosons $Z_{1 \text {,rec }}=Z_{1}$ and $Z_{2 \text {,rec }}=Z_{2}$. The respective particle groups are filled accordingly.

The CMS pairing (lepton_identification = 2) selects the OSSF lepton pair among all possible pairings (four, in the theoretical computation) that minimizes the invariant-mass difference to the $Z$-boson mass, $\mid m_{e^{-}} e^{+}$ $-m_{Z} \mid$. This pair is always identified as $Z_{1 \text {,rec }}$, while the remaining pair is defined as $Z_{2 \text {,rec. }}$.

\footnotetext{
${ }_{33}$ Note that the respective particle groups are also available within the $\mathrm{C}++$ code, see Appendix B.
}

This process provides an additional switch switch_off_shell to speed up the grid-generation phase when considering at least one of the $Z$ bosons to be far off-shell; it should not be used otherwise, see introduction of Sect. 5.2.4 for further details.

The process facilitates the following additional predefined dynamic scales (symbols in Table 5):

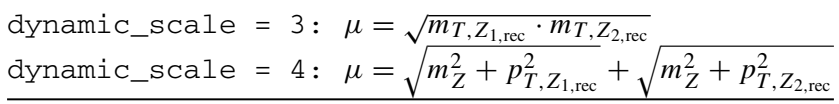

\subsubsection{5 ppeexnmnmx04 $\left(p p / p \bar{p} \rightarrow e^{-} e^{+} v_{\mu} \bar{v}_{\mu}\right)$}

Off-shell $Z Z$ production with $Z$-boson decays to leptons and neutrinos of different flavour ${ }^{34}$ includes the following predefined cuts:

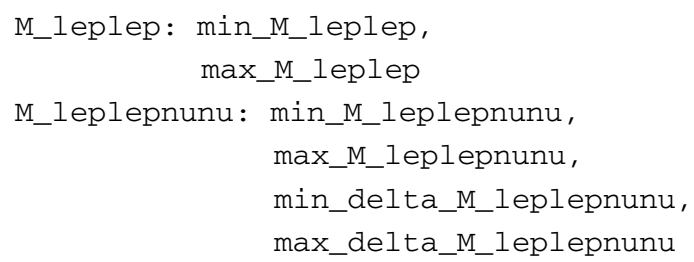

The process facilitates the following additional predefined dynamic scale (symbols in Table 5):

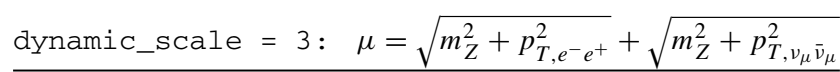

This process provides an additional switch switch_off_shell to speed up the grid-generation phase when considering at least one of the $Z$ bosons to be far off-shell; it should not be used otherwise, see introduction of Sect. 5.2.4 for further details.

\subsubsection{6 ppemxnmnex04 $\left(p p / p \bar{p} \rightarrow e^{-} \mu^{+} v_{\mu} \bar{v}_{e}\right)$}

Off-shell $W^{+} W^{-}$production $[36,37]$ with $W$-boson decays to DF leptons and the corresponding neutrinos ${ }^{35}$ includes the following predefined cuts:

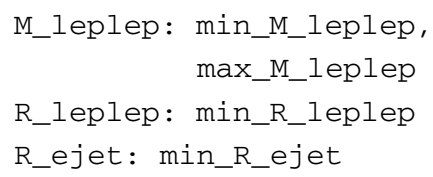

${ }^{34}$ Note that this process includes also $Z \gamma^{*}$ contributions with the offshell photon decaying to leptons, and $Z / \gamma^{*}$ production with a subsequent decay $Z / \gamma^{*} \rightarrow e^{-} e^{+} \nu_{\mu} \bar{v}_{\mu}$.

35 Note that this process includes also $Z / \gamma^{*}$ production with a subsequent decay $Z / \gamma^{*} \rightarrow e^{-} \mu^{+} v_{\mu} \bar{v}_{e}$. 


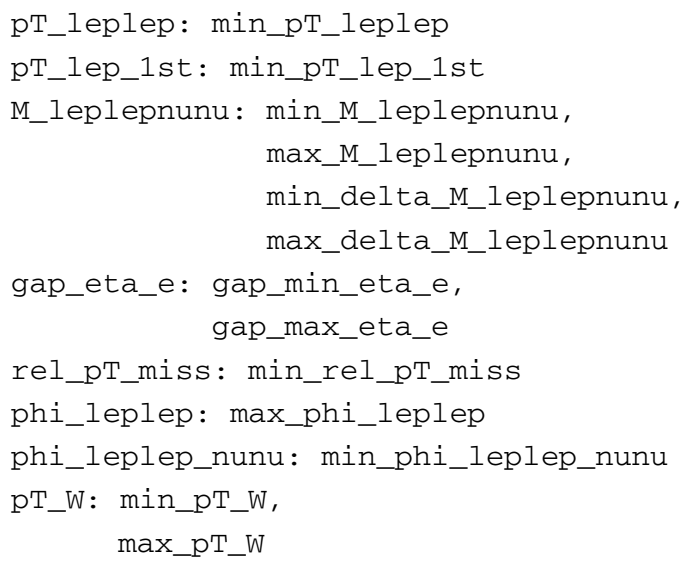

The process facilitates the following additional predefined dynamic scales (symbols in Table 5):

dynamic_scale $=3: \mu=\sqrt{m_{W}^{2}+p_{T, e^{-} \bar{v}_{e}}^{2}}+\sqrt{m_{W}^{2}+p_{T, \mu^{+} v_{\mu}}^{2}}$ dynamic_scale $=4: \mu=m_{T, e^{-} \bar{\nu}_{e}}+m_{T, \mu^{+}} v_{\mu}$

By default ( $f$ lavour_scheme $=0$ ) this process is computed in the 4FS, dropping all diagrams with final-state bottom quarks to remove the top-quark contamination. The 5FS can be chosen by setting flavour_scheme $=1$, where, however, the top-quark contamination is not removed from the results, since the recommended procedure is much more involved and requires several runs for the $5 \mathrm{FS}$, see introduction of Sect. 5.2.4 for further details.

\subsubsection{7 ppeexnenex04 $\left(p p / p \bar{p} \rightarrow e^{-} e^{+} v_{e} \bar{v}_{e}\right)$}

Off-shell $W^{+} W^{-}$and $Z Z$ production with decays to SF leptons and the corresponding neutrinos ${ }^{36}$ includes the following predefined cuts:

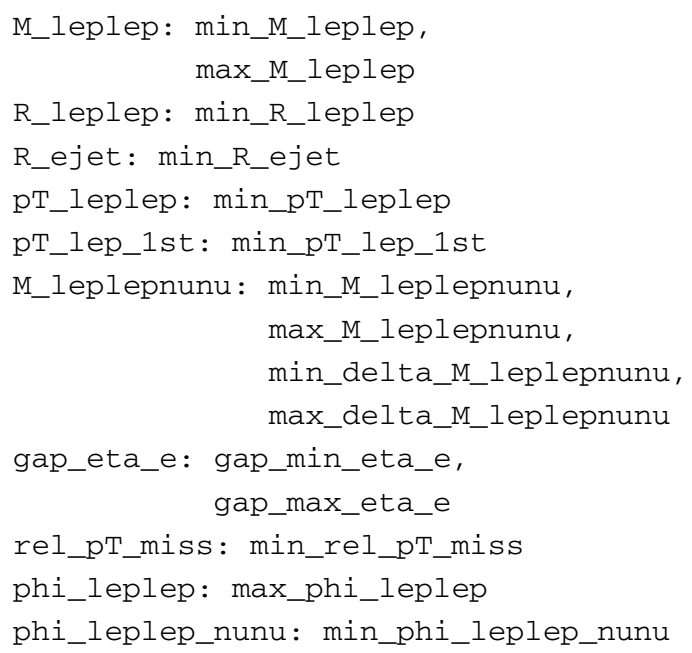

${ }^{36}$ Note that this process includes also $Z \gamma^{*}$ contributions with the offshell photon decaying to leptons, and $Z / \gamma^{*}$ production with a subsequent decay $Z / \gamma^{*} \rightarrow e^{-} e^{+} v_{e} \bar{v}_{e}$.

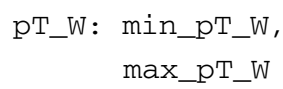

The process facilitates the following additional predefined dynamic scales (symbols in Table 5): $\begin{array}{ll}\text { dynamic_scale }=3: & \mu=\sqrt{m_{W}^{2}+p_{T, e^{-} \bar{v}_{e}}^{2}}+\sqrt{m_{W}^{2}+p_{T, e^{+} v_{e}}^{2}} \\ \text { dynamic_scale }=4: & \mu=m_{T, e^{-} \bar{v}_{e}}+m_{T, e^{+} v_{e}}\end{array}$

By default (flavour_scheme $=0$ ) this process is computed in the 4FS, dropping all diagrams with final-state bottom quarks to remove the top-quark contamination. The 5FS can be chosen by setting flavour_scheme $=1$, where, however, the top-quark contamination is not removed from the results, since the recommended procedure is much more involved and requires several runs for the $5 \mathrm{FS}$, see introduction of Sect. 5.2.4 for further details.

This process includes an additional switch switch_off_shell to speed up the grid-generation phase when considering at least one of the $Z$ bosons to be far off-shell; it should not be used otherwise, see introduction of Sect. 5.2.4 for further details.

\subsubsection{8 ppemexnmx04 $\left(p p / p \bar{p} \rightarrow e^{-} \mu^{-} e^{+} \bar{v}_{\mu}\right)$, ppeexmxnm04 $\left(p p / p \bar{p} \rightarrow e^{-} e^{+} \mu^{+} \nu_{\mu}\right)$}

Off-shell $W^{ \pm} Z$ production [38,39] with decays to one OSSF lepton pair, one DF lepton and one corresponding neutrino ${ }^{37}$ (DF channel) includes the following predefined cuts:

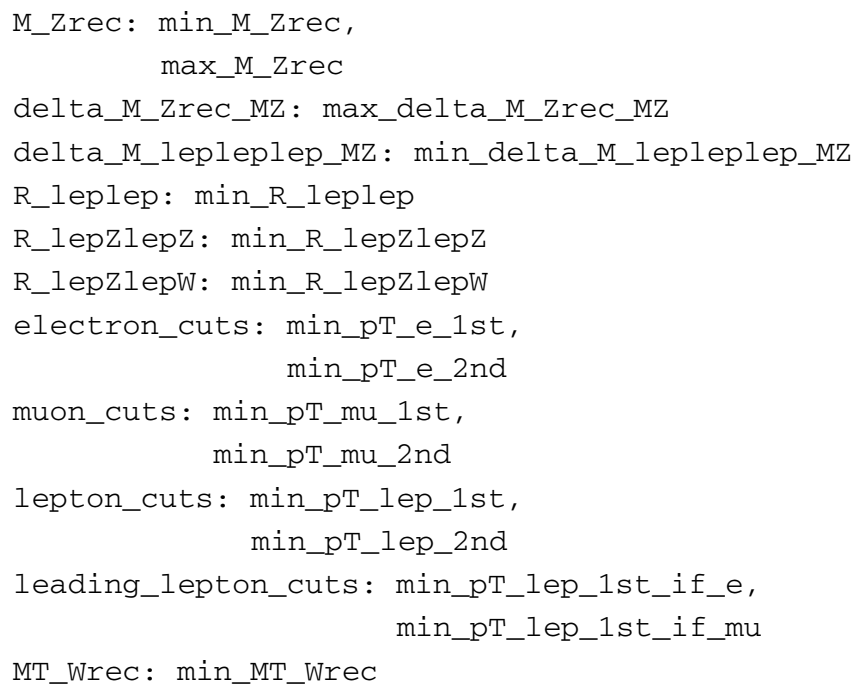

In the DF channel $W$ and $Z$ bosons can be unambiguously identified. In analogy to the SF case we define the following particle groups which can be accessed, e.g., in distributions:

\footnotetext{
37 Note that this process includes also $W^{-} / W^{+}$production with a subsequent decay $W \rightarrow e^{-} e^{+} \mu v_{\mu}$.
} 
Particle group Zrec contains the $Z$ boson, reconstructed by the two electrons, and Wrec the $W$ boson, defined by the muon and the neutrino. lepz contains the corresponding leptons of the $Z$ boson, ordered in their transverse momentum, and lepW the lepton of the $W$ boson.

The process facilitates the following additional predefined dynamic scales (symbols in Table 5):

dynamic_scale $=3: \mu=\frac{1}{2}\left(\sqrt{m_{Z}^{2}+p_{T, Z_{\mathrm{rec}}}}+\sqrt{m_{W}^{2}+p_{T, W_{\mathrm{rec}}^{ \pm}}^{2}}\right)$
dynamic_scale $=4: \mu=\frac{1}{2}\left(m_{T, Z_{\mathrm{rec}}}+m_{T, W_{\mathrm{rec}}}\right)$

\subsubsection{9 ppeeexnex04 ( $\left.p p / p \bar{p} \rightarrow e^{-} e^{-} e^{+} \bar{v}_{e}\right)$,} ppeexexne04 $\left(p p / p \bar{p} \rightarrow e^{-} e^{+} e^{+} v_{e}\right)$

Off-shell $W^{ \pm} Z$ production $[38,39]$ with decays to three SF leptons and one corresponding neutrino ${ }^{38}$ (SF channel) includes the following predefined cuts:

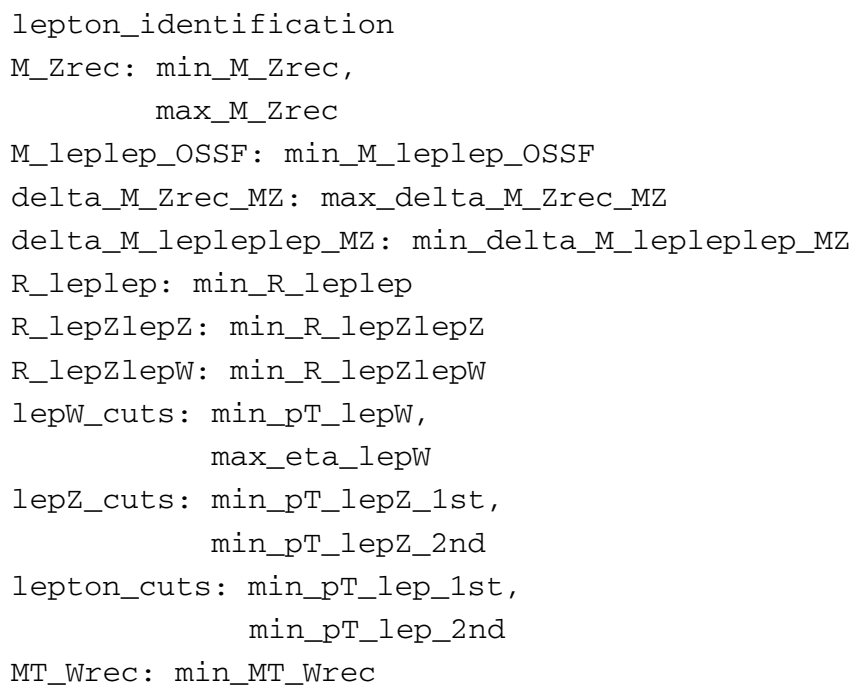

Since this process features three SF leptons, two of which have the same charge, and one neutrino, a-priori the leptons are not unambiguously associated with the decays of the $Z$ and $W$ bosons. However, the experimental analyses often rely on cuts specific to (reconstructed) $Z$ and $W$ bosons. Hence, in the SF channel an identification procedure is required to unambiguously assign one OSSF lepton pair to the $Z$ boson as well as the remaining lepton and the neutrino to the $W$ boson. The parameter lepton_identification switches between two such predefined identification procedures, as used by ATLAS and CMS. In both cases four new particle groups are defined (see Sect. 5.1.1.4 and the

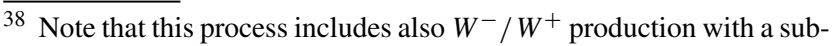
sequent decay $W \rightarrow e^{-} e^{+} e v_{e}$.
}

related Table 3 for the standard particle groups) to make them accessible in cuts, scales and distributions: Particle group Zrec contains the OSSF lepton pair that is reconstructed as a $Z$ boson, labelled $Z_{\text {rec }}$. Wrec contains the lepton and the neutrino that are reconstructed as a $W$ boson, labelled $W_{\text {rec }}$. lepz is filled with the leptons corresponding to the reconstructed $Z$ boson in the standard $p_{T}$-ordering, and lepW with the lepton assigned to the $W$ boson. By definition each of the other particle groups effectively contains only one particle, whereas lepz contains two particles. Examples for the usage of these particle groups can be found in the file distribution. dat of this process. Furthermore, many of the predefined cuts, e.g. delta_M_Zrec_MZ: max_delta_M_Zrec_MZ, MT_Wrec: min_MT_Wrec

or R_lepZlepW: min_R_lepZlepW, use the respective particle groups, which are filled according to the chosen value of the switch lepton_identification. ${ }^{39}$

If lepton_identification = 0 is set, the respective particle groups are not filled and thus cannot be used to define distributions. Also dynamic scales and cuts depending on the identification must not be used in this case. Finally, we outline the predefined pairing prescriptions:

ATLAS applies the so-called resonant-shape procedure [107] (lepton_identification = 1), where the assignment that maximizes the estimator

$$
P=\left|\frac{1}{m_{e^{-} e^{+}}^{2}-m_{Z}^{2}+i \Gamma_{Z} m_{Z}}\right|^{2} \cdot\left|\frac{1}{m_{e^{ \pm \prime} v_{e}}^{2}-m_{W}^{2}+i \Gamma_{W} m_{W}}\right|^{2}
$$

is chosen to identify $Z_{\mathrm{rec}}=Z$ and $W_{\mathrm{rec}}=W$, and the respective particles groups are filled accordingly. ${ }^{40}$

The CMS pairing (lepton_identification = 2) simply chooses the OSSF lepton pair that minimizes the invariant-mass difference to the $Z$-boson mass, i.e. $\mid m_{e^{-}} e^{+}$ $-m_{Z} \mid$. This pair is identified as $Z_{\text {rec }}$, and $W_{\text {rec }}$ and the other particle groups are assigned accordingly.

The process facilitates the following additional predefined dynamic scales (symbols in Table 5):

dynamic_scale $=3: \mu=\frac{1}{2}\left(\sqrt{m_{Z}^{2}+p_{T, Z_{\mathrm{rec}}}^{2}}+\sqrt{m_{W}^{2}+p_{T, W_{\mathrm{rec}}}^{ \pm}}\right)$
dynamic_scale $=4: \mu=\frac{1}{2}\left(m_{T, Z_{\mathrm{rec}}}+m_{T, W_{\mathrm{rec}}}^{ \pm}\right)$

\footnotetext{
${ }^{39}$ Note that the respective particle groups are also available within the $\mathrm{C}++$ code, see Appendix B.

40 We note that this definition requires the knowledge of the complete momentum of the neutrino. This variable can, of course, be used in the theoretical calculation, but cannot be directly extracted in the experimental analysis, where it must be reconstructed with the Monte Carlo.
} 


\section{Phenomenological results}

In this section we present results on integrated cross sections for all processes available in the first MATRIX release. They are reported at LO, NLO and NNLO to study the impact of QCD radiative corrections. We also discuss the impact of the loop-induced $g g$ contribution on the NNLO cross section, if applicable. The results in this section are obtained with the MATRIX default setup for each of these processes. Their purpose is both to provide benchmark numbers for all processes that can be evaluated with MATRIX, and to give a reference for the user: These benchmark results can be reproduced (on a statistical level) if no changes are applied to the default input cards (except for turning on the corresponding perturbative orders and the targeted precision the user is interested in).

\subsection{Settings}

We consider proton-proton collisions at the $13 \mathrm{TeV}$ LHC. In terms of the input of the weak parameters, the $G_{\mu}$ scheme is employed: When considering leptonic final state, which are always produced via off-shell EW vector bosons, we use the complex-mass scheme [108] throughout, i.e. we use complex $W$ - and $Z$-boson masses and define the EW mixing angle as $\cos \theta_{W}^{2}=\left(m_{W}^{2}-i \Gamma_{W} m_{W}\right) /\left(m_{Z}^{2}-i \Gamma_{Z} m_{Z}\right)$ and $\alpha=\sqrt{2} G_{\mu} m_{W}^{2} \sin ^{2} \theta_{W} / \pi$, using the PDG [105] values $G_{F}=1.16639 \times 10^{-5} \mathrm{GeV}^{-2}, m_{W}=80.385 \mathrm{GeV}$, $\Gamma_{W}=2.0854 \mathrm{GeV}, m_{Z}=91.1876 \mathrm{GeV}$ and $\Gamma_{Z}=$ $2.4952 \mathrm{GeV}$. Furthermore, we set $m_{H}=125 \mathrm{GeV}$ and $\Gamma_{H}=$ $0.00407 \mathrm{GeV}$. When considering on-shell single-boson production or on-shell production of heavy-boson pairs, the masses of the weak vector bosons and the weak mixing angle are consistently kept real by setting $\Gamma_{W}=\Gamma_{Z}=0$, and we also use a real Higgs boson mass, i.e. $\Gamma_{H}=0$. The number of heavy-quark flavours depends on the applied flavour scheme. As outlined in Sect. 5.2.4, all processes involving $W^{+} W^{-}$contributions use the 4FS as default to consistently remove top-quark contamination by dropping the (separately IR finite) partonic processes with real bottomquark emissions. In the 4FS we use the on-shell bottom mass $m_{b}=4.92 \mathrm{GeV}$. All other processes apply the 5FS with a vanishing bottom mass $m_{b}=0$. The top quark is treated as massive and unstable throughout, and we set $m_{t}=173.2 \mathrm{GeV}$ as well as $\Gamma_{t}=1.44262 \mathrm{GeV}^{41}$ We use the consistent NNPDF3.0 [109] set of parton distributions (PDFs) with $n_{f}=4$ or $n_{f}=5$ active quark flavours. $\mathrm{N}^{n} \mathrm{LO}$

\footnotetext{
$\overline{41}$ Massive top-quark contributions are neglected in the virtual two-loop corrections, but are kept anywhere else in the computations. Besides the fact that massive quark contributions in the two-loop amplitudes are not available and at the edge of current technology, their numerical effect can be expected to be negligible in most cases.
}

$(n=0,1,2)$ predictions are obtained by using PDFs at the same perturbative order and the evolution of $\alpha_{\mathrm{S}}$ at $(n+1)$ loop order, as provided by the corresponding PDF set. ${ }^{42}$ The CKM matrix is set to unity except for the production of a single (on- or off-shell) $W^{ \pm}$boson. In that case we use the PDG SM values as reported in Ref. [105]:

$$
V_{\mathrm{CKM}}=\left(\begin{array}{lll}
V_{u d} & V_{u s} & V_{u b} \\
V_{c d} & V_{c s} & V_{c b} \\
V_{t d} & V_{t s} & V_{t b}
\end{array}\right)=\left(\begin{array}{ccc}
0.97417 & 0.2248 & 0.00409 \\
0.22 & 0.995 & 0.0405 \\
0.0082 & 0.04 & 1.009
\end{array}\right) .
$$

Our reference choice $\mu_{0}$ for renormalization $\left(\mu_{R}\right)$ and factorization $\left(\mu_{F}\right)$ scales as well as the set of cuts applied in our default setups depend on the individual process. Both are reported when discussing the results in the upcoming section. Uncertainties from missing higher-order contributions are estimated in the usual way by independently varying $\mu_{R}$ and $\mu_{F}$ in the range $0.5 \mu_{0} \leq \mu_{R}, \mu_{F} \leq 2 \mu_{0}$, with the constraint $0.5 \leq \mu_{R} / \mu_{F} \leq 2$. Unless specified otherwise, jets are defined by the anti- $k_{T}$ clustering algorithm, $R=0.4$, $p_{T, j}>25 \mathrm{GeV}$ and $\left|\eta_{j}\right|<4.5$.

\subsection{Cross-section predictions}

Reference LO, NLO and NNLO predictions of the integrated cross sections for all processes that are available in MATRIX are reported in Table 6 . Note that the processes under consideration feature cross sections that may differ by several orders of magnitude, starting from a few fb up to several nb.

Two results are reported at NNLO: $\sigma_{\mathrm{NNLO}}^{r_{\text {cut }}}$ denotes the NNLO cross section at a fixed $r_{\text {cut }}$ value; the default $r_{\text {cut }}=$ $0.15 \%$ is used throughout for our reference results. Our best prediction is denoted as $\sigma_{\mathrm{NNLO}}^{\text {extrapolated }}$, and it is determined by the $r_{\text {cut }} \rightarrow 0$ extrapolation of the $r_{\text {cut }}$ dependence between $r_{\text {cut }}=0.15 \%$ and $r_{\text {cut }}=1 \%$ (see Sect. 7 for details). Both NNLO predictions are provided at the end of every MATRIX run, and for each process the results in Table 6 are taken from the same MATRIX run. The relative uncertainties, automatically computed by the code, refer to scale variations, as defined in Sect. $6.1 .^{43}$ The numerical uncertainty is reported in round brackets for all our predic-

\footnotetext{
42 More precisely, in the 5FS we use NNPDF30_10_as_0118, NNPDF30 nlo as 0118, and NNPDF30 nnlo as 0118 at LO, NLO, and NNLO, corresponding to $\alpha_{\mathrm{S}}^{(5 F)}\left(m_{Z}\right)=0.1180$ throughout. In the 4FS we use NNPDF30_1o_as_0118_nf_4, Footnote 42 continued

NNPDF30_nlo_as_0118_nf_4, and NNPDF30_nnlo_as_ 0118_ nf_4 at LO, NLO, and NNLO, corresponding to $\alpha_{\mathrm{S}}^{(4 F)}\left(m_{Z}\right)=0.1136,0.1123$, and 0.1123 , respectively.

43 The automatic evaluation of PDF uncertainties is not supported in the first release of MATRIX.
} 
tions. For $\sigma_{\text {NNLO }}^{\text {extrapolated }}$ this uncertainty is obtained by combining the statistical uncertainty from Monte Carlo integration with the systematic uncertainty induced by the $r_{\text {cut }}$ dependence.

Besides results at LO, NLO and NNLO accuracy, a separate column refers to the absolute (and relative) size of the loop-induced $g g$ component $\sigma_{\text {loop }}\left(\sigma_{\text {loop }} / \Delta \sigma_{\mathrm{NNLO}}^{\text {ext }}\right)$ of the NNLO corrections, where applicable. The absolute size of the NNLO contributions for the extrapolated result is defined as $\Delta \sigma_{\mathrm{NNLO}}^{\text {ext }}=\sigma_{\mathrm{NNLO}}^{\text {extrapolated }}-\sigma_{\mathrm{NLO}}$, where $\sigma_{\mathrm{NLO}}$ is computed with NLO PDFs. Two additional columns refer to the relative size of the radiative corrections in terms of $K$ factors at NLO and NNLO, defined as

$K_{\mathrm{NLO}}=\frac{\sigma_{\mathrm{NLO}}}{\sigma_{\mathrm{LO}}}$ and $K_{\mathrm{NNLO}}=\frac{\sigma_{\mathrm{NNLO}}}{\sigma_{\mathrm{NLO}}}$.

The latter are computed from our best NNLO predictions, i.e. the extrapolated NNLO results.

For all production processes involving massive on-shell bosons $\left(H, Z, W^{ \pm}, W^{+} W^{-}\right.$and $Z Z$ production), Table 6 reports fully inclusive cross sections, i. e. no phase-space cuts are applied. For all remaining processes, phase-space cuts are applied on the final-state leptons, neutrinos and photons in order to simulate a realistic selection in a fiducial volume. The respective sets of cuts for each of these processes are discussed below. For detailed studies of phenomenological results we refer to dedicated publications on the respective processes. We restrict ourselves to summarizing basic features of the calculations and the overall effect of the higherorder QCD corrections.

\section{Higgs boson production}

Radiative corrections to Higgs boson production are known to be particularly large. The corresponding cross sections in Table 6 have been computed with fixed renormalization and factorization scales set to $\mu_{0}=m_{H}$. The results have been checked to be in perfect agreement within the quoted numerical uncertainty with the analytic code SUSHI [110]. We find $K_{\mathrm{NLO}}=1.96$ and $K_{\mathrm{NNLO}}=1.32$ for the NLO and NNLO $K$ factors, respectively. As it is well known [35], scale variations significantly decrease upon inclusion of radiative corrections, but at LO and NLO they do not reflect the actual size of missing higher-order contributions.

\section{Drell-Yan production}

On-shell Drell-Yan production is another well-studied process, and it was the first hadron-collider process for which NNLO corrections were computed [2,3]. The results reported in Table 6 are obtained with renormalization and factorization scales set to $\mu_{0}=m_{Z}$ and $\mu_{0}=m_{W}$ for $p p \rightarrow Z$ and $p p \rightarrow W^{ \pm}$, respectively. The same fixed scales are applied to the corresponding off-shell processes. The Drell-Yan cross section is more than three orders of magnitude larger than the one of Higgs boson production, and the impact of radiative corrections is known to be smaller: NLO corrections increase the LO result by about $25 \%$, and NNLO corrections amount to a further $+3 \%$ effect. $p p \rightarrow Z$ has been checked numerically against the analytic result of Refs. [2,3], and we have validated the CKM implementation by finding full agreement at the level of the numerical errors for $p p \rightarrow e^{ \pm} v$ with FEWZ [53] and DYNNLO [12]. ${ }^{44}$

The cross sections of the charged-current and neutralcurrent Drell-Yan processes correspond in a first approximation to the on-shell $W$ or $Z$ production cross sections times the respective leptonic branching ratios. Consequently, they decrease by at least one order of magnitude with respect to the on-shell case.

The following sets of cuts, which are also summarized in Table 7, are applied to these processes: Every final-state lepton is required to have a minimum transverse momentum of $p_{T, \ell}>25 \mathrm{GeV}$ and a maximal pseudo-rapidity $\left|\eta_{\ell}\right|<2.47$. Neutrinos originating from a $W$-boson decay are restricted by a minimal requirement on the total missing transverse momentum, $p_{T}^{\text {miss }}>20 \mathrm{GeV}$. Additionally, we require $66 \mathrm{GeV}<m_{\ell^{-} \ell^{+}}<116 \mathrm{GeV}$ for the invariant mass of the two leptons in $p p \rightarrow e^{-} e^{+}$. The lower cut separates the leptons to avoid singularities arising from the photon-mediated contributions to this process. The process $p p \rightarrow v_{e} \bar{v}_{e}$, which is only relevant as a technical check, is calculated without any phase-space cuts. We find that, except for the $p p \rightarrow e^{-} e^{+}$process, which is affected by perturbative instabilities as discussed below, the off-shell contributions and additional phase-space cuts hardly have any effect on radiative corrections, which remain $K_{\mathrm{NLO}} \sim 1.25$ and $K_{\mathrm{NNLO}} \sim 1.03$ as in the on-shell case. The ratio of $W^{-}$and $W^{+}$cross sections does not significantly differ between on-shell and off-shell $W^{ \pm}$production: We find roughly $\sigma_{W^{-}} / \sigma_{W^{+}} \sim 0.75$, essentially independent of the perturbative order.

We note that the $p p \rightarrow e^{-} e^{+}$process has a peculiarly large $r_{\text {cut }}$ dependence at NNLO, similar to the processes involving final-state photons, thereby leading to a rather large systematic uncertainty. The large $r_{\text {cut }}$ dependence is due to the choice of symmetric $p_{T}$ thresholds on the leptons, which causes perturbative instabilities in the integrated cross section, as first observed in Ref. [111] (for a recent discussion of this problem, see Ref. [112]). ${ }^{45}$ However, choosing asym-

\footnotetext{
${ }^{44}$ More precisely, FEWZ and MATRIX agree at the permille level (see Sect. 7), and the agreement between DYNNLO and MATRIX at fixed $r_{\text {cut }}$ values is at the same level.

45 We note that a finite $r_{\text {cut }}$ leads to a smoother behaviour of the NNLO cross section in the delicate region of symmetric cuts (see e.g. Ref. [113]). One should, however, keep in mind that $r_{\text {cut }}$ is not to be understood as a tuning parameter. The smoother behaviour is a simple consequence of the fact that a finite $r_{\text {cut }}$ resolves the delicate phasespace region in less detail, thus smearing the unphysical behaviour of
} 
Table 6 Integrated cross sections for all available processes in MATRIX using the default setups

\begin{tabular}{|c|c|c|c|c|c|c|c|}
\hline $\begin{array}{l}\text { Process } \\
(\$\{\text { process_id }\})\end{array}$ & $\sigma_{\mathrm{LO}}$ & $\sigma_{\mathrm{NLO}}$ & $\begin{array}{l}\sigma_{\text {loop }} \\
\left(\sigma_{\text {loop }} / \Delta \sigma_{\mathrm{NNLO}}^{\text {ext }}\right)\end{array}$ & $\sigma_{\mathrm{NNLO}}^{r_{\mathrm{cut}}}$ & $\sigma_{\mathrm{NNLO}}^{\text {extrapolated }}$ & $K_{\mathrm{NLO}}(\%)$ & $K_{\mathrm{NNLO}}(\%)$ \\
\hline $\begin{array}{l}p p \rightarrow H \\
(\mathrm{pph} 21)\end{array}$ & $15.42(0)_{-17 \%}^{+22 \%} \mathrm{pb}$ & $30.26(1)_{-15 \%}^{+20 \%} \mathrm{pb}$ & - & $39.93(3)_{-10 \%}^{+11 \%} \mathrm{pb}$ & $39.93(3)_{-10 \%}^{+11 \%} \mathrm{pb}$ & +96.2 & +32.0 \\
\hline $\begin{array}{l}p p \rightarrow Z \\
(\mathrm{ppz} 01)\end{array}$ & $43.32(0)_{-13 \%}^{+12 \%} \mathrm{nb}$ & $54.20(1)_{-4.9 \%}^{+3.1 \%} \mathrm{nb}$ & - & $56.01(3)_{-1.1 \%}^{+0.84 \%} \mathrm{nb}$ & $55.99(3)_{-1.1 \%}^{+0.84 \%} \mathrm{nb}$ & +25.1 & +3.31 \\
\hline $\begin{array}{l}p p \rightarrow W^{-} \\
(\text {ppw01) }\end{array}$ & $60.15(0)_{-14 \%}^{+13 \%} \mathrm{nb}$ & $75.95(2)_{-5.3 \%}^{+3.3 \%} \mathrm{nb}$ & - & $78.36(3)_{-1.2 \%}^{+0.98 \%} \mathrm{nb}$ & $78.33(8)_{-1.2 \%}^{+0.98 \%} \mathrm{nb}$ & +26.3 & +3.14 \\
\hline $\begin{array}{l}p p \rightarrow W^{+} \\
(\mathrm{ppwx} 01)\end{array}$ & $81.28(1)_{-14 \%}^{+13 \%} \mathrm{nb}$ & $102.2(0)_{-5.3 \%}^{+3.4 \%} \mathrm{nb}$ & - & $105.8(1)_{-1.3 \%}^{+0.93 \%} \mathrm{nb}$ & $105.8(1)_{-1.3 \%}^{+0.93 \%} \mathrm{nb}$ & +25.7 & +3.52 \\
\hline $\begin{array}{l}p p \rightarrow e^{-} e^{+} \\
(\text {ppeex02) }\end{array}$ & $592.8(1)_{-14 \%}^{+14 \%} \mathrm{pb}$ & $699.7(2)_{-4.5 \%}^{+2.9 \%} \mathrm{pb}$ & - & $728.4(3)_{-0.72 \%}^{+0.48 \%} \mathrm{pb}$ & $732.7(3.4)_{-0.79 \%}^{+0.43 \%} \mathrm{pb}$ & +18.0 & +4.72 \\
\hline $\begin{array}{l}p p \rightarrow v_{e} \bar{v}_{e} \\
(\text { ppnenex02) }\end{array}$ & $2876(0)_{-13 \%}^{+12 \%} \mathrm{pb}$ & $3585(1)_{-4.9 \%}^{+3.0 \%} \mathrm{pb}$ & - & $3705(2)_{-1.1 \%}^{+0.86 \%} \mathrm{pb}$ & $3710(2)_{-1.1 \%}^{+0.85 \%} \mathrm{pb}$ & +24.6 & +3.48 \\
\hline $\begin{array}{l}p p \rightarrow e^{-} \bar{v}_{e} \\
(\text { ppenex02) }\end{array}$ & $2972(0)_{-15 \%}^{+14 \%} \mathrm{pb}$ & $3674(1)_{-5.2 \%}^{+3.1 \%} \mathrm{pb}$ & - & $3772(2)_{-0.94 \%}^{+0.89 \%} \mathrm{pb}$ & $3768(3)_{-0.93 \%}^{+0.90 \%} \mathrm{pb}$ & +23.6 & +2.57 \\
\hline $\begin{array}{l}p p \rightarrow e^{+} v_{e} \\
(\text { ppexne02) }\end{array}$ & $3964(0)_{-14 \%}^{+14 \%} \mathrm{pb}$ & $4855(1)_{-5.1 \%}^{+3.0 \%} \mathrm{pb}$ & - & $4986(2)_{-0.95 \%}^{+0.88 \%} \mathrm{pb}$ & $4986(3)_{-0.95 \%}^{+0.88 \%} \mathrm{pb}$ & +22.5 & +2.70 \\
\hline $\begin{array}{l}p p \rightarrow \gamma \gamma \\
(\text { ppaa 02) }\end{array}$ & $5.592(1)_{-11 \%}^{+10 \%} \mathrm{pb}$ & $25.75(1)_{-7.5 \%}^{+8.8 \%} \mathrm{pb}$ & $\begin{array}{l}2.534(1)_{-17 \%}^{+24 \%} \mathrm{pb} \\
(17.4 \%)\end{array}$ & $40.86(2)_{-7.2 \%}^{+8.7 \%} \mathrm{pb}$ & $40.28(30)_{-7.0 \%}^{+8.7 \%} \mathrm{pb}$ & +361 & +56.4 \\
\hline $\begin{array}{l}p p \rightarrow e^{-} e^{+} \gamma \\
(\text { ppeexa03) }\end{array}$ & $1469(0)_{-12 \%}^{+12 \%} \mathrm{fb}$ & $2119(1)_{-4.6 \%}^{+2.9 \%} \mathrm{fb}$ & $\begin{array}{l}16.02(1)_{-18 \%}^{+24 \%} \mathrm{fb} \\
(8.14 \%)\end{array}$ & $2326(1)_{-1.3 \%}^{+1.2 \%} \mathrm{fb}$ & $2316(5)_{-1.2 \%}^{+1.1 \%} \mathrm{fb}$ & +44.3 & +9.29 \\
\hline $\begin{array}{l}p p \rightarrow v_{e} \bar{v}_{e} \gamma \\
\text { (ppnenexa03) }\end{array}$ & $63.61(1)_{-3.5 \%}^{+2.7 \%} \mathrm{fb}$ & $98.75(2)_{-2.7 \%}^{+3.3 \%} \mathrm{fb}$ & $\begin{array}{l}2.559(2)_{-19 \%}^{+26 \%} \mathrm{fb} \\
(17.3 \%)\end{array}$ & $114.7(1)_{-2.6 \%}^{+3.2 \%} \mathrm{fb}$ & $113.5(6)_{-2.4 \%}^{+2.9 \%} \mathrm{fb}$ & +55.2 & +15.0 \\
\hline $\begin{array}{l}p p \rightarrow e^{-} \bar{v}_{e} \gamma \\
(\text { ppenexa03) }\end{array}$ & $726.1(1)_{-12 \%}^{+11 \%} \mathrm{fb}$ & $1850(1)_{-5.3 \%}^{+6.6 \%} \mathrm{fb}$ & - & $2286(1)_{-3.7 \%}^{+4.0 \%} \mathrm{fb}$ & $2256(15)_{-3.5 \%}^{+3.7 \%} \mathrm{fb}$ & +155 & +22.0 \\
\hline $\begin{array}{l}p p \rightarrow e^{+} v_{e} \gamma \\
(\text { ppexnea 03) }\end{array}$ & $861.7(1)_{-11 \%}^{+10 \%} \mathrm{fb}$ & $2187(1)_{-5.3 \%}^{+6.6 \%} \mathrm{fb}$ & - & $2707(3)_{-3.8 \%}^{+4.1 \%} \mathrm{fb}$ & $2671(35)_{-3.6 \%}^{+3.8 \%} \mathrm{fb}$ & +154 & +22.1 \\
\hline $\begin{array}{l}p p \rightarrow Z Z \\
(p p z z 02)\end{array}$ & $9.845(1)_{-6.3 \%}^{+5.2 \%} \mathrm{pb}$ & $14.10(0)_{-2.4 \%}^{+2.9 \%} \mathrm{pb}$ & $\begin{array}{l}1.361(1)_{-19 \%}^{+25 \%} \mathrm{pb} \\
(52.9 \%)\end{array}$ & $16.68(1)_{-2.6 \%}^{+3.2 \%} \mathrm{pb}$ & $16.67(1)_{-2.6 \%}^{+3.2 \%} \mathrm{pb}$ & +43.3 & +18.2 \\
\hline $\begin{array}{l}p p \rightarrow W^{+} W^{-} \\
(\mathrm{ppw \times w02)}\end{array}$ & $66.64(1)_{-6.7 \%}^{+5.7 \%} \mathrm{pb}$ & $103.2(0)_{-3.1 \%}^{+3.9 \%} \mathrm{pb}$ & $\begin{array}{l}4.091(3)_{-19 \%}^{+27 \%} \mathrm{pb} \\
(29.5 \%)\end{array}$ & $117.1(1)_{-2.2 \%}^{+2.5 \%} \mathrm{pb}$ & $117.1(1)_{-2.2 \%}^{+2.5 \%} \mathrm{pb}$ & +54.9 & +13.4 \\
\hline $\begin{array}{l}p p \rightarrow e^{-} \mu^{-} e^{+} \mu^{+} \\
(\text {ppemexmx } 04)\end{array}$ & $11.34(0)_{-7.3 \%}^{+6.3 \%} \mathrm{fb}$ & $16.87(0)_{-2.5 \%}^{+3.0 \%} \mathrm{fb}$ & $\begin{array}{l}1.971(1)_{-18 \%}^{+25 \%} \mathrm{fb} \\
(57.6 \%)\end{array}$ & $20.30(1)_{-2.9 \%}^{+3.5 \%} \mathrm{fb}$ & $20.30(1)_{-2.9 \%}^{+3.5 \%} \mathrm{fb}$ & +48.8 & +20.3 \\
\hline $\begin{array}{l}p p \rightarrow e^{-} e^{-} e^{+} e^{+} \\
(\text {ppeeexex04) }\end{array}$ & $5.781(1)_{-7.4 \%}^{+6.3 \%} \mathrm{fb}$ & $8.623(3)_{-2.5 \%}^{+3.1 \%} \mathrm{fb}$ & $\begin{array}{l}0.9941(4)_{-18 \%}^{+25 \%} \mathrm{fb} \\
(56.9 \%)\end{array}$ & $10.37(1)_{-3.0 \%}^{+3.5 \%} \mathrm{fb}$ & $10.37(1)_{-3.0 \%}^{+3.5 \%} \mathrm{fb}$ & +49.2 & +20.2 \\
\hline $\begin{array}{l}p p \rightarrow e^{-} e^{+} v_{\mu} \bar{v}_{\mu} \\
(\text { ppeexnmnmx } 04)\end{array}$ & $22.34(0)_{-6.4 \%}^{+5.3 \%} \mathrm{fb}$ & $33.90(1)_{-2.7 \%}^{+3.3 \%} \mathrm{fb}$ & $\begin{array}{l}3.212(1)_{-19 \%}^{+25 \%} \mathrm{fb} \\
(49.6 \%)\end{array}$ & $40.39(2)_{-2.8 \%}^{+3.5 \%} \mathrm{fb}$ & $40.38(2)_{-2.8 \%}^{+3.5 \%} \mathrm{fb}$ & +51.7 & +19.1 \\
\hline $\begin{array}{l}p p \rightarrow e^{-} \mu^{+} v_{\mu} \bar{v}_{e} \\
(\text { ppemxnmnex 04) }\end{array}$ & $232.9(0)_{-7.6 \%}^{+6.6 \%} \mathrm{fb}$ & $236.1(1)_{-2.4 \%}^{+2.8 \%} \mathrm{fb}$ & $\begin{array}{l}26.93(1)_{-19 \%}^{+27 \%} \mathrm{fb} \\
(94.3 \%)\end{array}$ & $264.7(1)_{-1.4 \%}^{+2.2 \%} \mathrm{fb}$ & $264.6(2)_{-1.4 \%}^{+2.2 \%} \mathrm{fb}$ & +1.34 & +12.1 \\
\hline $\begin{array}{l}p p \rightarrow e^{-} e^{+} v_{e} \bar{v}_{e} \\
(\text { ppeexnenex04) }\end{array}$ & $115.0(0)_{-7.3 \%}^{+6.3 \%} \mathrm{fb}$ & $203.4(1)_{-3.8 \%}^{+4.7 \%} \mathrm{fb}$ & $\begin{array}{l}12.62(1)_{-19 \%}^{+26 \%} \mathrm{fb} \\
(33.8 \%)\end{array}$ & $240.8(1)_{-3.0 \%}^{+3.4 \%} \mathrm{fb}$ & $240.7(1)_{-3.0 \%}^{+3.4 \%} \mathrm{fb}$ & +76.9 & +18.4 \\
\hline $\begin{array}{l}p p \rightarrow e^{-} \mu^{-} e^{+} \bar{v}_{\mu} \\
(\text { ppemexnmx 04) }\end{array}$ & $11.50(0)_{-6.8 \%}^{+5.7 \%} \mathrm{fb}$ & $23.55(1)_{-4.5 \%}^{+5.5 \%} \mathrm{fb}$ & - & $26.17(1)_{-2.1 \%}^{+2.2 \%} \mathrm{fb}$ & $26.17(2)_{-2.1 \%}^{+2.2 \%} \mathrm{fb}$ & +105 & +11.1 \\
\hline $\begin{array}{l}p p \rightarrow e^{-} e^{-} e^{+} \bar{v}_{e} \\
(\text { ppeeexnex04) }\end{array}$ & $11.53(0)_{-6.8 \%}^{+5.7 \%} \mathrm{fb}$ & $23.63(1)_{-4.5 \%}^{+5.5 \%} \mathrm{fb}$ & - & $26.27(1)_{-2.1 \%}^{+2.3 \%} \mathrm{fb}$ & $26.25(2)_{-2.1 \%}^{+2.3 \%} \mathrm{fb}$ & +105 & +11.1 \\
\hline
\end{tabular}


Table 6 continued

\begin{tabular}{|c|c|c|c|c|c|c|c|}
\hline $\begin{array}{l}\text { Process } \\
(\$\{\text { process_id }\})\end{array}$ & $\sigma_{\mathrm{LO}}$ & $\sigma_{\mathrm{NLO}}$ & $\begin{array}{l}\sigma_{\text {loop }} \\
\left(\sigma_{\text {loop }} / \Delta \sigma_{\mathrm{NNLO}}^{\text {ext }}\right)\end{array}$ & $\sigma_{\mathrm{NNLO}}^{r_{\text {cut }}}$ & $\sigma_{\mathrm{NNLO}}^{\text {extrapolated }}$ & $K_{\mathrm{NLO}}(\%)$ & $K_{\mathrm{NNLO}}(\%)$ \\
\hline $\begin{array}{l}p p \rightarrow e^{-} e^{+} \mu^{+} v_{\mu} \\
(\text { ppeexmxnm04) }\end{array}$ & $17.33(0)_{-6.3 \%}^{+5.3 \%} \mathrm{fb}$ & $34.14(1)_{-4.3 \%}^{+5.3 \%} \mathrm{fb}$ & - & $37.74(2)_{-2.0 \%}^{+2.2 \%} \mathrm{fb}$ & $37.74(4)_{-2.0 \%}^{+2.2 \%} \mathrm{fb}$ & +97.0 & +10.6 \\
\hline $\begin{array}{l}p p \rightarrow e^{-} e^{+} e^{+} v_{e} \\
(\text { ppeexexne04) }\end{array}$ & $17.37(0)_{-6.3 \%}^{+5.3 \%} \mathrm{fb}$ & $34.21(2)_{-4.3 \%}^{+5.3 \%} \mathrm{fb}$ & - & $37.85(2)_{-2.0 \%}^{+2.3 \%} \mathrm{fb}$ & $37.84(3)_{-2.0 \%}^{+2.3 \%} \mathrm{fb}$ & +96.9 & +10.6 \\
\hline
\end{tabular}

Table 7 Default setup of fiducial cuts for $Z, W^{ \pm}$and $\gamma \gamma$ production processes

\begin{tabular}{llll}
\hline & $p p \rightarrow e^{-} e^{+}$ & $p p \rightarrow e^{-} \bar{v}_{e} / p p \rightarrow e^{+} \nu_{e}$ & $p p \rightarrow \gamma \gamma$ \\
\hline Lepton cuts & $p_{T, \ell}>25 \mathrm{GeV},\left|\eta_{\ell}\right|<2.47$ & $p_{T, \ell}>25 \mathrm{GeV},\left|\eta_{\ell}\right|<2.47$ & - \\
& $66 \mathrm{GeV}<m_{\ell^{-} \ell^{+}}<116 \mathrm{GeV}$ & $p_{T, \gamma_{1}}>40 \mathrm{GeV}, p_{T, \gamma_{2}}>25 \mathrm{GeV}$ \\
Photon cuts & - & - & $\left|\eta_{\gamma}\right|<2.5$ \\
& & $20 \mathrm{GeV}<m_{\gamma \gamma}<250 \mathrm{GeV}$ \\
Neutrino cuts & - & $p_{T}^{\text {miss }}>20 \mathrm{GeV}$ & - \\
Photon isolation & - & - & Frixione isolation with \\
& & $n$ & $n=1, \varepsilon=0.5$ and $\delta_{0}=0.4$
\end{tabular}

Table 8 Default setup of fiducial cuts for $Z \gamma$ and $W^{ \pm} \gamma$ production processes

\begin{tabular}{llll}
\hline & $p p \rightarrow e^{-} e^{+} \gamma$ & $p p \rightarrow v_{e} \bar{v}_{e}$ & $p p \rightarrow e^{-} \bar{v}_{e} / p p \rightarrow e^{+} v_{e} \gamma$ \\
\hline Lepton cuts & $p_{T, \ell}>25 \mathrm{GeV},\left|\eta_{\ell}\right|<2.47$ & - & $p_{T, \ell}>25 \mathrm{GeV},\left|\eta_{\ell}\right|<2.47$ \\
& $m_{\ell-\ell^{+}}>40 \mathrm{GeV}$ & $p_{T, \gamma}>15 \mathrm{GeV},\left|\eta_{\gamma}\right|<2.37$ \\
Photon cuts & $p_{T, \gamma}>15 \mathrm{GeV},\left|\eta_{\gamma}\right|<2.37$ & $p_{T, \gamma}>100 \mathrm{GeV},\left|\eta_{\gamma}\right|<2.37$ & $p_{T}^{\text {miss }}>35 \mathrm{GeV}$ \\
Neutrino cuts & - & $p_{T}^{\text {miss }}>90 \mathrm{GeV}$ & $\Delta R_{\ell j}>0.3, \Delta R_{\gamma j}>0.3$, \\
Separation cuts & $\Delta R_{\ell j}>0.3, \Delta R_{\gamma j}>0.3$, & $\Delta R_{\gamma j}>0.3$ & $\Delta R_{\ell \gamma}>0.7$ \\
Photon isolation & $\Delta R_{\ell \gamma}>0.7$ & Frixione isolation with $n=1, \varepsilon=0.5$ and $\delta_{0}=0.4$ & \\
Jet definition & & Anti- $k_{T}$ algorithm with $R=0.4 ; p_{T, j}>30 \mathrm{GeV},\left|\eta_{j}\right|<4.4$
\end{tabular}

metric $p_{T}$ cuts on the harder and softer lepton does not reduce this dependence significantly. On the contrary, we find that if the asymmetric cuts are separately applied on the negatively and positively charged leptons (instead of applying them on the harder and softer lepton) the ensuing $r_{\text {cut }}$ behaviour is extremely flat and therefore a small $r_{\text {cut }} \rightarrow 0$ extrapolation uncertainty is obtained. The $r_{\text {cut }}$ behaviour of the $p p \rightarrow e^{-} e^{+}$process is discussed in Sect. 7, where we also present a comparison of our results with FEWZ. We stress that more accurate results for this process can be obtained through the setting of switch_qT_accuracy $=1$ in

the fixed-order cross section. A reliable perturbative prediction in the region of symmetric cuts can only be obtained through a dedicated resummation procedure. the file parameter. dat by using a minimal value of $r_{\text {cut }}=0.05 \%$ (default is $r_{\text {cut }}=0.15 \%$ ) for the extrapolation range, see Sect. 5.2.2.3.

\section{Diphoton and vector-boson plus photon production}

For diphoton production we choose the invariant mass of the photon pair as the central scale, i.e. $\mu_{0}=m_{\gamma \gamma}$, Frixione isolation with $n=1, \varepsilon=0.5$ and $\delta_{0}=0.4$ (see Eq. (3)), and the following fiducial cuts, which are also summarized in Table 7: The isolated photons are required to have a pseudorapidity $\left|\eta_{\gamma}\right|<2.5$, and the transverse momentum of the (sub)leading photon must fulfil $p_{T, \gamma}>40(25) \mathrm{GeV}$. Further, we require $20 \mathrm{GeV}<m_{\gamma \gamma}<250 \mathrm{GeV}$ for the invariant mass of the two photons. Our predictions show the importance of QCD corrections for this process: With $K_{\mathrm{NLO}}=4.61$ and 
$K_{\mathrm{NNLO}}=1.56$, higher-order effects are enormous [28,29] and not at all reflected by the estimated scale uncertainties at lower orders. This process entails a loop-induced $g g$ component in the NNLO cross section. With only a $17 \%$ contribution to the NNLO correction it has a rather moderate impact though. Our results have been compared to those obtained with the $2 \gamma \mathrm{NNLO}$ code at fixed values of $r_{\text {cut }}$, and agreement has been found at the level of $0.5 \%$.

Next, we consider the associated production of an offshell vector boson with a photon, i.e. the leptonic final states $e^{-} e^{+} \gamma / \nu_{e} \bar{v}_{e} \gamma$ (summarized as $Z \gamma$ production) and $e^{+} \nu_{e} \gamma / e^{-} \bar{\nu}_{e} \gamma$ (summarized as $W \gamma$ production). ${ }^{46}$ Our setup is adopted from Ref. [31]: The dynamic scale $\mu_{0}=$ $\sqrt{m_{V}^{2}+p_{T, \gamma}^{2}}$ is chosen as central value for both renormalization and factorization scales, where $m_{V}=m_{Z}$ for $Z \gamma$ and $m_{V}=m_{W}$ for $W \gamma$ production. As for diphoton production, Frixione isolation with $n=1, \varepsilon=0.5$ and $\delta_{0}=0.4$ (see Eq. (3)) is used to identify photons. The fiducial cuts include standard cuts on leptons, photons and the missing transverse momentum, as well as lepton-photon, lepton-jet and photon-jet separations in $R$. The numerical values of these cuts are summarized in Table 8 . The $Z \gamma$ processes feature large corrections, $K_{\mathrm{NLO}}\left(K_{\mathrm{NNLO}}\right)=1.44(1.09)$ for $p p \rightarrow e^{-} e^{+} \gamma$ and $K_{\mathrm{NLO}}\left(K_{\mathrm{NNLO}}\right)=1.55(1.15)$ for $p p \rightarrow$ $v_{e} \bar{v}_{e} \gamma$. For $W \gamma$ production, radiative corrections are known to be huge due to a radiation zero at LO [114]: At NLO the $W^{ \pm} \gamma$ cross section is increased by more than $+150 \%$, and the NNLO corrections have a further effect of $+22 \%$. The ratio between the $W^{-} \gamma$ and $W^{+} \gamma$ cross sections is roughly $\sigma_{W^{-} \gamma} / \sigma_{W^{+} \gamma} \sim 0.75$, widely independent of the perturbative order and very similar to the ratio of the charged Drell-Yan processes.

One should bear in mind that all processes with isolated photons in the final state have a relatively large uncertainty at NNLO ( $\sim 0.5-1 \%)$ even after the $r_{\text {cut }} \rightarrow 0$ extrapolation (which in the default setup is based on the $r_{\text {cut }}$ dependence between $r_{\text {cut }}=0.15 \%$ and $r_{\text {cut }}=1 \%$ ). More accurate results can be obtained by setting switch_qT_accuracy $=1$ in the file parameter. dat, which uses a minimal value of $r_{\text {cut }}=0.05 \%$ for the extrapolation range, see Sect. 5.2.3. We refer to the discussion in Sect. 7 for details.

\section{Vector-boson pair production}

The on-shell $Z Z$ and $W^{+} W^{-}$results in Table 6 correspond to the inclusive cross sections of Ref. [33] and Ref. [36], respectively, with an updated set of input parameters. We have explicitly checked that MATRIX reproduces the results of Refs. $[33,36]$ when adjusting the setup accordingly. Con-

\footnotetext{
46 We note again that $Z \gamma$ and $W \gamma$ are only used as shorthand notations here. The full amplitudes for the leptonic final states are used throughout without any approximation, including off-shell effects and spin correlations.
}

sistent with these studies, we have used fixed renormalization and factorization scales of $\mu_{0}=m_{Z}$ and $\mu_{0}=m_{W}$ for $Z Z$ and $W^{+} W^{-}$production, respectively. Radiative corrections are large for both processes: The NLO corrections amount to $+43 \%$ at NLO and still $+18 \%$ at NNLO in the case of $Z Z$ production, where a bit more than half of the NNLO corrections originates from the loop-induced $g g$ channel, though. The predicted $W^{+} W^{-}$cross section receives NLO corrections of $+55 \%$, and NNLO corrections lead to a further increase by $+13 \%$, a third of which results from the loop-induced $g g$ contribution. For both processes the corrections exceed by far the perturbative uncertainties estimated by scale variations at lower orders. This is caused, in part, by the additional contribution from the $g g$ component, which is not covered by NLO scale variations. The purely gluoninduced NLO corrections to the $g g$ channel, which are part of a complete $\mathrm{N}^{3} \mathrm{LO}$ calculation, have been computed in Refs. $[115,116]$.

Several leptonic channels originate from off-shell $Z Z$ production. They involve the SF and DF four-lepton channels, $4 \ell$ and $2 \ell 2 \ell^{\prime}$, respectively, which have been studied at NNLO QCD in Ref. [34]. On the other hand, one of the $Z$ bosons may decay to two neutrinos instead. In that case the SF channel is defined as the one where the neutrino flavour matches the lepton flavour $(2 \ell 2 v)$, while the DF flavour channel is defined as the one where the lepton and neutrino flavours are different $\left(2 \ell 2 v^{\prime}\right) .{ }^{47}$ The SF $2 \ell 2 v$ final state is special since it receives contributions from both resonant $Z Z$ and $W^{+} W^{-}$ sub-topologies, which mix the two processes. From an experimental viewpoint, in the $Z Z$ or $W^{+} W^{-}$analyses the two production mechanisms are isolated by using suitable cuts that enhance the respective process in its signal region. Since we include all resonant and non-resonant topologies leading to such final states, our computation of $2 \ell 2 v$ is applicable to both $Z Z$ and $W^{+} W^{-}$studies by simply applying the corresponding cuts. NNLO cross sections for the $2 \ell 2 v$ and $2 \ell 2 v^{\prime}$ channels are reported here for the first time. A detailed study of these processes will be presented elsewhere.

For the off-shell $Z Z$ processes we use the setup of Ref. [34]: The renormalization and factorization scales are fixed to $\mu_{0}=m_{Z}$. The fiducial cuts are summarized in Table 9. They involve standard transverse-momentum and rapidity thresholds for the leptons, and a lepton-lepton separation in $R$. In the $2 \ell 2 \ell^{\prime}$ channel, the invariant mass of OSSF lepton pairs is required to be in a mass window around the $Z$ peak. In the $4 \ell$ channel, there are two possible combinations of OSSF lepton pairs that can be associated with the par-

\footnotetext{
47 We note that both final states contain an OSSF lepton pair and (possibly) missing transverse momentum from the two neutrinos that cannot be detected. Our distinction into SF and DF final states is motivated more by the underlying technical calculations than by their phenomenology in this case.
} 
Table 9 Default setup of fiducial cuts for $Z Z$ and $Z Z / W^{+} W^{-}$production processes

\begin{tabular}{lll}
\hline & $p p \rightarrow e^{-} \mu^{-} e^{+} \mu^{+} / p p \rightarrow e^{-} e^{-} e^{+} e^{+}$ & $p p \rightarrow e^{-} e^{+} v_{e} \bar{\nu}_{e} / p p \rightarrow e^{-} e^{+} v_{\mu} \bar{v}_{\mu}$ \\
\hline Lepton cuts & $p_{T, \ell}>7 \mathrm{GeV},\left|\eta_{\ell}\right|<2.7$ & $p_{T, \ell}>7 \mathrm{GeV},\left|\eta_{\ell}\right|<2.7$ \\
& $66 \mathrm{GeV}<m_{\ell^{-} \ell^{+}}<116 \mathrm{GeV}$ & $66 \mathrm{GeV}<m_{\ell^{-} \ell^{+}}<116 \mathrm{GeV}$ \\
Neutrino cuts & - & $p_{T}^{\text {miss }}>30 \mathrm{GeV}$ \\
Separation cuts & $\Delta R_{\ell \ell}>0.2$ & - \\
Jet definition & \multicolumn{2}{c}{ Anti- $k_{T}$ algorithm with $R=0.4 ;$} \\
\hline
\end{tabular}

Table 10 Default setup of fiducial cuts for $W^{+} W^{-}$and $W^{ \pm} Z$ production processes

\begin{tabular}{lll}
\hline & $p p \rightarrow e^{-} \mu^{+} v_{\mu} \bar{v}_{e}$ & $p p \rightarrow \ell^{\prime \pm} \nu_{\ell^{\prime}} \ell^{+} \ell^{-}, \quad \ell, \ell^{\prime} \in\{e, \mu\}$ \\
\hline Lepton cuts & $p_{T, \ell_{1}}>25 \mathrm{GeV}, p_{T, \ell_{2}}>20 \mathrm{GeV}$ & $p_{T, \ell_{\mathrm{z}}}>15 \mathrm{GeV}, p_{T, \ell_{\mathrm{w}}}>20 \mathrm{GeV}$ \\
& $\left|\eta_{e}\right|<2.47,\left|\eta_{e}\right| \notin[1.37 ; 1.52]$ & $\left|\eta_{\ell}\right|<2.5$ \\
& $\left|\eta_{\mu}\right|<2.4, m_{\ell^{-} \ell^{+}}>10 \mathrm{GeV}$ & $\left|m_{\ell_{\mathrm{z}} \ell_{\mathrm{z}}}-m_{Z}\right|<10 \mathrm{GeV}$ \\
Neutrino cuts & $p_{T}^{\text {miss }}>20 \mathrm{GeV}, p_{T}^{\text {miss,rel }}>15 \mathrm{GeV}$ & $m_{T, W}>30 \mathrm{GeV}$ \\
Separation cuts & $\Delta R_{\ell \ell}>0.1$ & $\Delta R_{\ell_{\mathrm{z}} \ell_{\mathrm{z}}}>0.2, \Delta R_{\ell_{\mathrm{z}} \ell_{\mathrm{w}}}>0.3$ \\
Jet cuts & $N_{\text {jets }}=0 \quad-$ \\
Jet definition & \multicolumn{2}{c}{ Anti- $k_{T}$ algorithm with $R=0.4 ; p_{T, j}>25 \mathrm{GeV},\left|\eta_{j}\right|<4.5$} \\
\hline
\end{tabular}

ent $Z$ bosons. We choose the combination which minimizes $\left|m_{\ell^{-} \ell^{+}}-m_{Z}\right|+\left|m_{\ell^{-\prime} \ell^{+\prime}}-m_{Z}\right|$, see Sect. 5.2.4.4 for details, and apply the invariant mass cuts only on the corresponding lepton pairs. Since no dedicated phenomenological studies of the $2 \ell 2 v / 2 \ell 2 v^{\prime}$ signatures at NNLO exist, we simply adopt the $2 \ell 2 \ell^{\prime}$ setup, while removing the $R$ separation of the leptons and adding a loose cut on the missing transverse energy of the neutrinos. This choice provides a generic benchmark scenario for these processes.

Comparing the SF $4 \ell$ process $p p \rightarrow e^{-} e^{-} e^{+} e^{+}$and the DF $2 \ell 2 \ell^{\prime}$ process $p p \rightarrow e^{-} \mu^{-} e^{+} \mu^{+}$in Table 6 , it is obvious that they give very similar results, taking into account the relative combinatorial factor of one-half in the SF channel (if the two SF channels $p p \rightarrow e^{-} e^{-} e^{+} e^{+}$and $p p \rightarrow \mu^{-} \mu^{-} \mu^{+} \mu^{+}$ are added, DF and SF channels would be of the same size again). It is not surprising that the $K$ factors for the $4 \ell, 2 \ell 2 \ell^{\prime}$ and $2 \ell 2 v^{\prime}$ channels are very close, given the fact that very similar cuts are applied and that the dominant contribution results from resonant $Z Z$ production in all these processes. The NLO corrections amount to roughly $+50 \%$, and the cross sections are increased by further $\sim+20 \%$ at NNLO, i.e. radiative corrections in the fiducial regions are even a bit larger than for the inclusive $\mathrm{ZZ}$ cross section. For the $4 \ell$ and $2 \ell 2 \ell^{\prime}$ channels, the loop-induced $g g$ component has a slightly bigger impact ( $\sim 57 \%$ of the NNLO corrections) in the fiducial phase space than in the fully inclusive case ( $\sim 53 \%)$, whereas it contributes a bit less for the $2 \ell 2 v^{\prime}$ channel $(\sim 50 \%)$.

The SF $2 \ell 2 v$ channel, on the other hand, shows a rather different behaviour due to the large impact of its $W^{+} W^{-}$ topologies, which are expected to dominate by about an order of magnitude due to the involved EW couplings and branching ratios. Under the quite loose cuts, compared to a dedi- cated $Z Z \rightarrow \ell \ell \nu v$ selection, the cross section at LO is still about a factor of five larger than for the $2 \ell 2 v^{\prime}$ process, due to the dominance of $W^{+} W^{-}$contributions. This cross section receives somewhat larger corrections than the "pure" $Z Z$ processes, namely $K_{\mathrm{NLO}}=1.77$ and $K_{\mathrm{NNLO}}=1.18$, where the $g g$ component contributes $33 \%$ of the NNLO corrections, comparable to on-shell $W^{+} W^{-}$production.

The off-shell $W^{+} W^{-}$process with DF leptons $\left(\ell v \ell^{\prime} v^{\prime}\right)$, namely $p p \rightarrow e^{-} \mu^{+} v_{\mu} \bar{v}_{e}$, has been studied at NNLO in Ref. [37]. We adopt the fixed scale choice of $\mu_{0}=m_{W}$ and the fiducial cuts used in that study. The latter are summarized in Table 10: Besides standard cuts like transverse momentum thresholds, rapidity ranges and different isolation criteria, the selection cut with the largest impact on the size of higher-order corrections is a jet veto, which is required in $W^{+} W^{-}$analyses to suppress top-quark backgrounds. As a consequence of the jet veto, and in contrast to the inclusive $W^{+} W^{-}$cross section, the fiducial cross section receives very small radiative corrections, only $+1.3 \%$ at NLO. The NNLO corrections amount to $+12 \%$, but they are almost entirely due to the loop-induced $g g$ component. This component (at its leading order, which in terms of power counting belongs to the NNLO corrections of the complete process) has Born-level kinematics and is thus not affected by the jet veto, whereas real-radiation corrections are significantly suppressed. However, higher-order corrections to the $g g$ contribution are affected by the jet veto, i.e. similar to the radiative corrections to the $q \bar{q}$ channel, they are significantly reduced with respect to an inclusive calculation. Hence, due to the suppression of radiative corrections by the jet veto, and the fact that no further new channels open up beyond NNLO, scale variations should provide a reasonable estimate of the uncertainties due to yet un-calculated higher-order QCD con- 
tributions. The purely gluon-induced NLO corrections to the $g g$ channel have been computed in Ref. [116].

With $W^{ \pm} Z$ production [38,39], the last diboson process has recently been computed at NNLO accuracy. Four different processes with three leptons and one neutrino are associated with $W^{ \pm} Z$ production: $W^{-} Z$ and $W^{+} Z$ production can each be split into a SF and a DF channel. Since these processes have charged final states, no loop-induced gg component contributes at NNLO. Following Ref. [39] we set $\mu_{0}=\left(m_{Z}+m_{W}\right) / 2$ for the central value of renormalization and factorization scales and use the fiducial cuts summarized in Table 10: The lepton transverse-momentum thresholds distinguish between leptons associated with the $Z$ - and the $W$-boson decays. The lepton pair associated with the $Z$-boson decay is required to have an invariant mass close to the $Z$-boson mass, and the transverse mass of the $W$ boson, defined through the lepton associated with the $W$ boson decay and the transverse missing-energy vector (see Appendix A), is restricted from below. Furthermore, leptons are required to be separated in $R$, where the separation depends on whether the respective leptons are both associated with the $Z$-boson decay or with the decays of two different heavy bosons. In the SF channel there is an ambiguity how to assign the leptons to the $Z$ - and $W$-boson decays, and we follow the resonant-shape identification procedure of Ref. [107] (see also Sect. 5.2.4.9 for details). Since Ref. [39] uses the most recent input parameters corresponding to the default MATRIX settings, the $13 \mathrm{TeV}$ results of the fiducial cross sections are exactly (within the numerical uncertainties) reproduced. Radiative corrections in that process are known to be large because of an approximate radiation zero [117] in the Born scattering amplitudes, which is broken beyond LO. We find $K_{\mathrm{NLO}}=2.05(1.97)$ and $K_{\mathrm{NNLO}}=1.11(1.11)$ for $W^{-} Z$ $\left(W^{+} Z\right.$ ), both for SF and DF channels. The $\sigma_{W^{-} Z} / \sigma_{W^{+} Z}$ ratio is about 0.69 , both at NLO and NNLO, i.e. slightly smaller than what is found for the $\sigma_{W^{-}} / \sigma_{W^{+}}$ratio in the chargedcurrent Drell-Yan process.

We conclude this section by discussing the performance of the code. In Table 11 we report the runtimes needed to obtain the LO, NLO and NNLO results of Table 6, converted to the time needed for a serial run on a single CPU core. The runtime estimates for achieving a statistical uncertainty of one permille, stated in the last column, facilitate a direct comparison among the various processes, and we find that the most time-consuming ones are those involving off-shell $V \gamma$ final states. We stress that the numerical uncertainties quoted in Table 11 are purely statistical: For processes featuring a large systematic uncertainty from the $r_{\text {cut }}$ extrapolation, the choice of the (purely statistical) target accuracy should be adapted to the size of the systematic uncertainties, where the latter can be reduced by setting switch_qT_accuracy $=1$.

\section{Systematic uncertainties of $q_{T}$ subtraction}

As pointed out before, NLO and NNLO cross sections computed with the $q_{T}$-subtraction formalism exhibit a residual dependence on the cut-off $r_{\text {cut }}$ in the slicing parameter $r=q_{T} / M$, where $q_{T}$ is the transverse momentum and $M$ the mass of the colourless system. This residual dependence is due to power-suppressed terms, which are left after the subtraction of the IR singular contribution at finite values of $r_{\text {cut }}$ and vanish only in the limit $r_{\text {cut }} \rightarrow 0$. The $r_{\text {cut }}$ dependence of the cross sections computed with the $q_{T}$-subtraction method has been discussed in some detail for the $W^{ \pm} \gamma$, offshell $W^{+} W^{-}$and off-shell $W^{ \pm} Z$ production processes in Refs. [31,37,39], to which we refer the reader interested in these specific processes. In the following, we study the systematic uncertainties of our results for a representative set of processes available in MATRIX, using the corresponding default setup of each process.

MATRIX performs an extrapolation $r_{\text {cut }} \rightarrow 0$ for total rates computed by means of the $q_{T}$-subtraction procedure, i.e. at NNLO, and possibly at NLO if the $q_{T}$-subtraction method is applied. A conservative estimate of the extrapolation uncertainty is included in the numerical error of this extrapolated result, which is considered our best prediction at the corresponding perturbative order and printed on screen at the end of each run. To perform the extrapolation, MATRIX automatically computes the cross section at fixed values of $r_{\text {cut }}$ in the interval $\left[r_{\text {cut }}^{\min } ; 1 \%\right]$ using steps of $0.01 \%$. Unless stated otherwise (see the process-specific information in Sect. 5.2), the default value MATRIX uses is $r_{\text {cut }}^{\mathrm{min}}=0.15 \%$. The extrapolation procedure, which is discussed below, has been tested to work extremely well at NLO, where $r_{\text {cut }}$-independent results are available. Note that already the cross section at the lowest calculated value $r_{\text {cut }}^{\mathrm{min}}=0.15 \%$ (actually also for higher $r_{\text {cut }}$ values up to at least $r_{\text {cut }} \sim 1 \%$ ) provides a very reasonable prediction in cases where the $r_{\text {cut }}$ dependence is small, and thus the result at $r_{\text {cut }}^{\min }$ is also printed on screen at the end of each run. A comparison of the extrapolated cross sec-

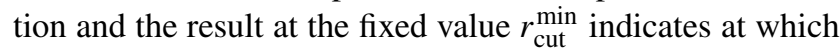
level of accuracy the fixed- $r_{\text {cut }}$ result can be trusted: In case of a significant $r_{\text {cut }}$ dependence of the total rate, we recommend to correct the kinematic distributions by the ratio $\sigma_{\mathrm{NNLO}}^{\text {extrapolated }} / \sigma_{\mathrm{NNLO}}^{r_{\text {cut }}}$. In the first release of MATRIX, distributions are indeed always calculated at $r_{\text {cut }}=r_{\text {cut }}^{\min }$. We note that such reweighting should not be applied to distributions that are trivial at LO: For example, the transversemomentum of the colourless system vanishes at LO, and its high- $p_{T}$ region is not affected by a finite $r_{\text {cut }}$ value. Given that we have not observed any significant $r_{\text {cut }}$ dependence of our NNLO results for kinematic distributions in various dedicated studies, where we have performed the extrapolation on a bin-by-bin basis (see, e.g., Ref. [39]), we consider 
Table 11 MATRIX runtime for integrated cross-section results in Table 6 . The relative uncertainty is entirely due to the numerical phase space integration. The runtimes correspond to the total time required for the production phase (pre-run and main-run) of the respective MATRIX runs if it was performed on a single CPU core. Automatic scale variations, $r_{\text {cut }}$ extrapolation and the computation of distributions has been included in these runs

\begin{tabular}{|c|c|c|c|c|}
\hline $\begin{array}{l}\text { Process } \\
(\$\{\text { process_id\}) }\end{array}$ & $\begin{array}{l}\text { LO runtime } \\
\text { (relative uncertainty) }\end{array}$ & $\begin{array}{l}\text { NLO runtime } \\
\text { (relative uncertainty) }\end{array}$ & $\begin{array}{l}\text { NNLO runtime } \\
\text { (relative uncertainty) }\end{array}$ & $\begin{array}{l}\text { NNLO runtime estimate } \\
\text { for } 10^{-3} \text { uncertainty }\end{array}$ \\
\hline $\begin{array}{l}p p \rightarrow H \\
(\mathrm{pph} 21)\end{array}$ & $\begin{array}{l}0 \mathrm{~d} 0 \mathrm{~h} 2 \mathrm{~min} \\
\left(1.5 \times 10^{-4}\right)\end{array}$ & $\begin{array}{l}0 \mathrm{~d} 0 \mathrm{~h} 12 \mathrm{~min} \\
\left(2.7 \times 10^{-4}\right)\end{array}$ & $\begin{array}{l}35 \mathrm{~d} 23 \mathrm{~h} 23 \mathrm{~min} \\
\left(7.2 \times 10^{-4}\right)\end{array}$ & $19 \mathrm{~d}$ \\
\hline $\begin{array}{l}p p \rightarrow Z \\
(\mathrm{ppz} 01)\end{array}$ & $\begin{array}{l}0 \mathrm{~d} 0 \mathrm{~h} 10 \mathrm{~min} \\
\left(8.2 \times 10^{-5}\right)\end{array}$ & $\begin{array}{l}0 \mathrm{~d} 0 \mathrm{~h} 16 \mathrm{~min} \\
\left(2.6 \times 10^{-4}\right)\end{array}$ & $\begin{array}{l}53 \mathrm{~d} 15 \mathrm{~h} 31 \mathrm{~min} \\
\left(4.6 \times 10^{-4}\right)\end{array}$ & $11 \mathrm{~d}$ \\
\hline $\begin{array}{l}p p \rightarrow W^{-} \\
(\mathrm{ppw} 01)\end{array}$ & $\begin{array}{l}0 \mathrm{~d} 0 \mathrm{~h} 7 \mathrm{~min} \\
\left(8.1 \times 10^{-5}\right)\end{array}$ & $\begin{array}{l}0 \mathrm{~d} 0 \mathrm{~h} 22 \mathrm{~min} \\
\left(2.6 \times 10^{-4}\right)\end{array}$ & $\begin{array}{l}50 \mathrm{~d} 17 \mathrm{~h} 29 \mathrm{~min} \\
\left(4.4 \times 10^{-4}\right)\end{array}$ & $10 \mathrm{~d}$ \\
\hline $\begin{array}{l}p p \rightarrow W^{+} \\
(\mathrm{ppw} \times 1)\end{array}$ & $\begin{array}{l}0 \mathrm{~d} 0 \mathrm{~h} 14 \mathrm{~min} \\
\left(8.1 \times 10^{-5}\right)\end{array}$ & $\begin{array}{l}0 \mathrm{~d} 0 \mathrm{~h} 24 \mathrm{~min} \\
\left(2.6 \times 10^{-4}\right)\end{array}$ & $\begin{array}{l}47 \mathrm{~d} 7 \mathrm{~h} 46 \mathrm{~min} \\
\left(4.9 \times 10^{-4}\right)\end{array}$ & $11 \mathrm{~d}$ \\
\hline $\begin{array}{l}p p \rightarrow e^{-} e^{+} \\
(\text {ppeex02) }\end{array}$ & $\begin{array}{l}0 \mathrm{~d} 0 \mathrm{~h} 48 \mathrm{~min} \\
\left(1.0 \times 10^{-4}\right)\end{array}$ & $\begin{array}{l}0 \mathrm{~d} 2 \mathrm{~h} 24 \mathrm{~min} \\
\left(2.8 \times 10^{-4}\right)\end{array}$ & $\begin{array}{l}173 \mathrm{~d} 20 \mathrm{~h} 36 \mathrm{~min} \\
\left(3.6 \times 10^{-4}\right)\end{array}$ & $22 \mathrm{~d}$ \\
\hline $\begin{array}{l}p p \rightarrow v_{e} \bar{v}_{e} \\
(\text { ppnenex } 02)\end{array}$ & $\begin{array}{l}0 \mathrm{~d} 1 \mathrm{~h} 31 \mathrm{~min} \\
\left(8.2 \times 10^{-5}\right)\end{array}$ & $\begin{array}{l}0 \mathrm{~d} 1 \mathrm{~h} 0 \mathrm{~min} \\
\left(2.5 \times 10^{-4}\right)\end{array}$ & $\begin{array}{l}89 \mathrm{~d} 18 \mathrm{~h} 17 \mathrm{~min} \\
\left(4.5 \times 10^{-4}\right)\end{array}$ & $18 \mathrm{~d}$ \\
\hline $\begin{array}{l}p p \rightarrow e^{-} \bar{v}_{e} \\
(\text { ppenex02) }\end{array}$ & $\begin{array}{l}0 \mathrm{~d} 1 \mathrm{~h} 46 \mathrm{~min} \\
\left(8.7 \times 10^{-5}\right)\end{array}$ & $\begin{array}{l}0 \mathrm{~d} 5 \mathrm{~h} 21 \mathrm{~min} \\
\left(2.2 \times 10^{-4}\right)\end{array}$ & $\begin{array}{l}114 \mathrm{~d} 2 \mathrm{~h} 18 \mathrm{~min} \\
\left(4.3 \times 10^{-4}\right)\end{array}$ & $21 \mathrm{~d}$ \\
\hline $\begin{array}{l}p p \rightarrow e^{+} v_{e} \\
(\text { ppexne02) }\end{array}$ & $\begin{array}{l}0 \mathrm{~d} 1 \mathrm{~h} 56 \mathrm{~min} \\
\left(8.5 \times 10^{-5}\right)\end{array}$ & $\begin{array}{l}0 \mathrm{~d} 3 \mathrm{~h} 43 \mathrm{~min} \\
\left(2.6 \times 10^{-4}\right)\end{array}$ & $\begin{array}{l}114 \mathrm{~d} 6 \mathrm{~h} 18 \mathrm{~min} \\
\left(4.6 \times 10^{-4}\right)\end{array}$ & $24 \mathrm{~d}$ \\
\hline $\begin{array}{l}p p \rightarrow \gamma \gamma \\
(\text { ppaa02) }\end{array}$ & $\begin{array}{l}0 \mathrm{~d} 1 \mathrm{~h} 13 \mathrm{~min} \\
\left(9.8 \times 10^{-5}\right)\end{array}$ & $\begin{array}{l}0 \mathrm{~d} 4 \mathrm{~h} 11 \mathrm{~min} \\
\left(2.8 \times 10^{-4}\right)\end{array}$ & $\begin{array}{l}27 \mathrm{~d} 17 \mathrm{~h} 7 \mathrm{~min} \\
\left(4.6 \times 10^{-4}\right)\end{array}$ & $6 \mathrm{~d}$ \\
\hline $\begin{array}{l}p p \rightarrow e^{-} e^{+} \gamma \\
(\text { ppeexa03) }\end{array}$ & $\begin{array}{l}0 \mathrm{~d} 17 \mathrm{~h} 55 \mathrm{~min} \\
\left(9.2 \times 10^{-5}\right)\end{array}$ & $\begin{array}{l}1 \mathrm{~d} 19 \mathrm{~h} 48 \mathrm{~min} \\
\left(2.8 \times 10^{-4}\right)\end{array}$ & $\begin{array}{l}1276 \mathrm{~d} 12 \mathrm{~h} 47 \mathrm{~min} \\
\left(3.6 \times 10^{-4}\right)\end{array}$ & $167 \mathrm{~d}$ \\
\hline $\begin{array}{l}p p \rightarrow v_{e} \bar{v}_{e} \gamma \\
(\text { ppnenexa03) }\end{array}$ & $\begin{array}{l}0 \mathrm{~d} 2 \mathrm{~h} 50 \mathrm{~min} \\
\left(8.7 \times 10^{-5}\right)\end{array}$ & $\begin{array}{l}0 \mathrm{~d} 8 \mathrm{~h} 59 \mathrm{~min} \\
\left(2.5 \times 10^{-4}\right)\end{array}$ & $\begin{array}{l}75 \mathrm{~d} 9 \mathrm{~h} 6 \mathrm{~min} \\
\left(4.7 \times 10^{-4}\right)\end{array}$ & $17 \mathrm{~d}$ \\
\hline $\begin{array}{l}p p \rightarrow e^{-} \bar{v}_{e} \gamma \\
(\text { ppenexa03) }\end{array}$ & $\begin{array}{l}0 \mathrm{~d} 22 \mathrm{~h} 18 \mathrm{~min} \\
\left(1.0 \times 10^{-4}\right)\end{array}$ & $\begin{array}{l}3 \mathrm{~d} 16 \mathrm{~h} 59 \mathrm{~min} \\
\left(3.2 \times 10^{-4}\right)\end{array}$ & $\begin{array}{l}1484 \mathrm{~d} 16 \mathrm{~h} 50 \mathrm{~min} \\
\left(4.0 \times 10^{-4}\right)\end{array}$ & $232 \mathrm{~d}$ \\
\hline $\begin{array}{l}p p \rightarrow e^{+} v_{e} \gamma \\
(\text { ppexnea03) }\end{array}$ & $\begin{array}{l}1 \mathrm{~d} 7 \mathrm{~h} 8 \mathrm{~min} \\
\left(9.6 \times 10^{-5}\right)\end{array}$ & $\begin{array}{l}6 \mathrm{~d} 8 \mathrm{~h} 7 \mathrm{~min} \\
\left(3.0 \times 10^{-4}\right)\end{array}$ & $\begin{array}{l}428 \mathrm{~d} 7 \mathrm{~h} 1 \mathrm{~min} \\
\left(1.0 \times 10^{-3}\right)\end{array}$ & $443 \mathrm{~d}$ \\
\hline $\begin{array}{l}p p \rightarrow Z Z \\
(\operatorname{ppzz} 02)\end{array}$ & $\begin{array}{l}0 \mathrm{~d} 1 \mathrm{~h} 44 \mathrm{~min} \\
\left(8.2 \times 10^{-5}\right)\end{array}$ & $\begin{array}{l}0 \mathrm{~d} 1 \mathrm{~h} 6 \mathrm{~min} \\
\left(2.4 \times 10^{-4}\right)\end{array}$ & $\begin{array}{l}132 \mathrm{~d} 19 \mathrm{~h} 37 \mathrm{~min} \\
\left(4.4 \times 10^{-4}\right)\end{array}$ & $25 \mathrm{~d}$ \\
\hline $\begin{array}{l}p p \rightarrow W^{+} W^{-} \\
(\text {ppwxw02) }\end{array}$ & $\begin{array}{l}0 \mathrm{~d} 1 \mathrm{~h} 23 \mathrm{~min} \\
\left(8.2 \times 10^{-5}\right)\end{array}$ & $\begin{array}{l}0 \mathrm{~d} 0 \mathrm{~h} 48 \mathrm{~min} \\
\left(2.5 \times 10^{-4}\right)\end{array}$ & $\begin{array}{l}69 \mathrm{~d} 20 \mathrm{~h} 49 \mathrm{~min} \\
\left(4.3 \times 10^{-4}\right)\end{array}$ & $13 \mathrm{~d}$ \\
\hline $\begin{array}{l}p p \rightarrow e^{-} \mu^{-} e^{+} \mu^{+} \\
(\text {ppemexmx04) }\end{array}$ & $\begin{array}{l}0 \mathrm{~d} 5 \mathrm{~h} 43 \mathrm{~min} \\
\left(8.2 \times 10^{-5}\right)\end{array}$ & $\begin{array}{l}0 \mathrm{~d} 4 \mathrm{~h} 32 \mathrm{~min} \\
\left(2.7 \times 10^{-4}\right)\end{array}$ & $\begin{array}{l}219 \mathrm{~d} 16 \mathrm{~h} 33 \mathrm{~min} \\
\left(4.5 \times 10^{-4}\right)\end{array}$ & $45 \mathrm{~d}$ \\
\hline $\begin{array}{l}p p \rightarrow e^{-} e^{-} e^{+} e^{+} \\
(\text {ppeeexex04) }\end{array}$ & $\begin{array}{l}0 \mathrm{~d} 11 \mathrm{~h} 34 \mathrm{~min} \\
\left(9.0 \times 10^{-5}\right)\end{array}$ & $\begin{array}{l}0 \mathrm{~d} 12 \mathrm{~h} 8 \mathrm{~min} \\
\left(3.4 \times 10^{-4}\right)\end{array}$ & $\begin{array}{l}742 \mathrm{~d} 13 \mathrm{~h} 37 \mathrm{~min} \\
\left(5.1 \times 10^{-4}\right)\end{array}$ & $193 \mathrm{~d}$ \\
\hline $\begin{array}{l}p p \rightarrow e^{-} e^{+} v_{\mu} \bar{v}_{\mu} \\
(\text { ppeexnmnmx } 04)\end{array}$ & $\begin{array}{l}0 \mathrm{~d} 6 \mathrm{~h} 33 \mathrm{~min} \\
\left(9.4 \times 10^{-5}\right)\end{array}$ & $\begin{array}{l}0 \mathrm{~d} 6 \mathrm{~h} 36 \mathrm{~min} \\
\left(2.7 \times 10^{-4}\right)\end{array}$ & $\begin{array}{l}158 \mathrm{~d} 13 \mathrm{~h} 40 \mathrm{~min} \\
\left(4.4 \times 10^{-4}\right)\end{array}$ & $31 \mathrm{~d}$ \\
\hline $\begin{array}{l}p p \rightarrow e^{-} \mu^{+} v_{\mu} \bar{v}_{e} \\
(\text { ppemxnmnex } 04)\end{array}$ & $\begin{array}{l}0 \mathrm{~d} 13 \mathrm{~h} 33 \mathrm{~min} \\
\left(9.2 \times 10^{-5}\right)\end{array}$ & $\begin{array}{l}1 \mathrm{~d} 22 \mathrm{~h} 9 \mathrm{~min} \\
\left(2.7 \times 10^{-4}\right)\end{array}$ & $\begin{array}{l}521 \mathrm{~d} 2 \mathrm{~h} 20 \mathrm{~min} \\
\left(4.8 \times 10^{-4}\right)\end{array}$ & $119 \mathrm{~d}$ \\
\hline $\begin{array}{l}p p \rightarrow e^{-} e^{+} v_{e} \bar{v}_{e} \\
(\text { ppeexnenex } 04)\end{array}$ & $\begin{array}{l}0 \mathrm{~d} 23 \mathrm{~h} 36 \mathrm{~min} \\
\left(8.2 \times 10^{-5}\right)\end{array}$ & $\begin{array}{l}0 \mathrm{~d} 17 \mathrm{~h} 46 \mathrm{~min} \\
\left(4.8 \times 10^{-4}\right)\end{array}$ & $\begin{array}{l}270 \mathrm{~d} 6 \mathrm{~h} 59 \mathrm{~min} \\
\left(4.4 \times 10^{-4}\right)\end{array}$ & $52 \mathrm{~d}$ \\
\hline $\begin{array}{l}p p \rightarrow e^{-} \mu^{-} e^{+} \bar{v}_{\mu} \\
(\text { ppemexnmx 04) }\end{array}$ & $\begin{array}{l}0 \mathrm{~d} 5 \mathrm{~h} 18 \mathrm{~min} \\
\left(1.0 \times 10^{-4}\right)\end{array}$ & $\begin{array}{l}0 \mathrm{~d} 5 \mathrm{~h} 15 \mathrm{~min} \\
\left(2.9 \times 10^{-4}\right)\end{array}$ & $\begin{array}{l}104 \mathrm{~d} 16 \mathrm{~h} 46 \mathrm{~min} \\
\left(4.3 \times 10^{-4}\right)\end{array}$ & $19 \mathrm{~d}$ \\
\hline
\end{tabular}


Table 11 continued

\begin{tabular}{|c|c|c|c|c|}
\hline $\begin{array}{l}\text { Process } \\
(\$\{\text { process_id\}) }\end{array}$ & $\begin{array}{l}\text { LO runtime } \\
\text { (relative uncertainty) }\end{array}$ & $\begin{array}{l}\text { NLO runtime } \\
\text { (relative uncertainty) }\end{array}$ & $\begin{array}{l}\text { NNLO runtime } \\
\text { (relative uncertainty) }\end{array}$ & $\begin{array}{l}\text { NNLO runtime estimate } \\
\text { for } 10^{-3} \text { uncertainty }\end{array}$ \\
\hline $\begin{array}{l}p p \rightarrow e^{-} e^{-} e^{+} \bar{v}_{e} \\
(\text { ppeeexnex04) }\end{array}$ & $\begin{array}{l}0 \mathrm{~d} 14 \mathrm{~h} 19 \mathrm{~min} \\
\left(8.3 \times 10^{-5}\right)\end{array}$ & $\begin{array}{l}0 \mathrm{~d} 14 \mathrm{~h} 56 \mathrm{~min} \\
\left(2.7 \times 10^{-4}\right)\end{array}$ & $\begin{array}{l}179 \mathrm{~d} 14 \mathrm{~h} 6 \mathrm{~min} \\
\left(4.7 \times 10^{-4}\right)\end{array}$ & $39 \mathrm{~d}$ \\
\hline $\begin{array}{l}p p \rightarrow e^{-} e^{+} \mu^{+} v_{\mu} \\
(\text { ppeexmxnm0 4) }\end{array}$ & $\begin{array}{l}0 \mathrm{~d} 10 \mathrm{~h} 32 \mathrm{~min} \\
\left(8.1 \times 10^{-5}\right)\end{array}$ & $\begin{array}{l}0 \mathrm{~d} 8 \mathrm{~h} 18 \mathrm{~min} \\
\left(2.6 \times 10^{-4}\right)\end{array}$ & $\begin{array}{l}104 \text { d } 17 \text { h } 58 \text { min } \\
\left(4.5 \times 10^{-4}\right)\end{array}$ & $21 \mathrm{~d}$ \\
\hline $\begin{array}{l}p p \rightarrow e^{-} e^{+} e^{+} v_{e} \\
(\text { ppeexexne04) }\end{array}$ & $\begin{array}{l}0 \mathrm{~d} 9 \mathrm{~h} 19 \mathrm{~min} \\
\left(1.0 \times 10^{-4}\right)\end{array}$ & $\begin{array}{l}0 \mathrm{~d} 13 \mathrm{~h} 11 \mathrm{~min} \\
\left(4.6 \times 10^{-4}\right)\end{array}$ & $\begin{array}{l}167 \mathrm{~d} 6 \mathrm{~h} 49 \mathrm{~min} \\
\left(5.1 \times 10^{-4}\right)\end{array}$ & $44 \mathrm{~d}$ \\
\hline
\end{tabular}

this reweighting procedure sufficiently accurate ${ }^{48}$, and leave a proper extrapolation procedure of distributions for a future update of MATRIX.

The $r_{\text {cut }} \rightarrow 0$ extrapolation of the cross section is obtained using a simple quadratic least $\chi^{2}$ fit. Such fit is repeated varying the upper bound of the fit interval, starting from a minimum upper bound of $0.5 \%$ ( $0.25 \%$ for dilepton production or processes involving photons with $r_{\text {cut }}^{\min }=0.15 \% ; 0.15 \%$ for the same processes with $r_{\text {cut }}^{\text {min }}=0.05 \%$ ), and the result with the lowest $\chi^{2} /$ degrees-of-freedom value is kept as the best fit. The extrapolation uncertainty is determined by comparing the result of the best fit with the results obtained by variations of the upper bound of the fit interval. To be conservative, a lower bound on this uncertainty is introduced, corresponding to half of the difference between the $r_{\text {cut }} \rightarrow 0$ result and the cross section at $r_{\text {cut }}^{\min }$. This extrapolation error is combined quadratically with the numerical error, which is determined by extrapolating also statistical errors at finite $r_{\text {cut }}$ values to $r_{\text {cut }}=0$.

Our results for the $r_{\text {cut }}$ dependence of a representative set of processes are shown in Fig. 2. Before commenting the various plots we provide some general explanation. The central values of the green bars represent the NNLO cross section calculated at the respective fixed $r_{\text {cut }}$ values, $r_{\text {cut }} \in[0.01 \% ; 1 \%]$ in steps of $0.01 \%$, and their sizes denote the numerical uncertainties. Our reference prediction, computed with the default MATRIX setup, is the $r_{\text {cut }} \rightarrow 0$ extrapolation obtained from the values $r_{\text {cut }} \geq 0.15 \%$, shown as a blue solid line. A vertical blue dotted line at $r_{\text {cut }}=0.15 \%$ indicates the lowest value used for this extrapolation. The blue uncertainty band is obtained by combining the numerical and extrapolation uncertainties and corresponds to the on-screen output of MATRIX. When the $r_{\text {cut }}$ dependence is strong, we also show the MATRIX result extrapolated from $r_{\text {cut }} \geq 0.05 \%$ values as a red solid line with a red band, with its uncertainty

\footnotetext{
48 We note that in the region close to kinematical boundaries the $r_{\text {cut }}$ dependence of the NNLO results is expected to be amplified by the presence of perturbative instabilities of Sudakov type [118]. In such regions, however, the predictivity of fixed-order computations is limited, and a resummation of the logarithmically enhanced contributions would be required.
}

computed analogously to the blue band. Where available, NNLO results obtained either from analytical calculations or from alternative NNLO numerical programs are reported as black lines, while the grey band shows their numerical integration error. All the results are reported as relative deviations from the reference prediction in percent.

We start our discussion from the first two plots in Fig. 2, which refer to the inclusive on-shell production of a Higgs (left) and a $Z$ boson (right), respectively. In both cases the NNLO cross sections turn out to be extremely stable with respect to $r_{\text {cut }}$ : Almost all fixed- $r_{\text {cut }}$ results deviate by less than one permille from the default $r_{\text {cut }} \rightarrow 0$ prediction, and all fixed- $r_{\text {cut }}$ results are compatible with the extrapolated result within their numerical uncertainties. The high stability with respect to $r_{\text {cut }}$ in these cases would justify choosing essentially any $r_{\text {cut }}$ value in the given range to provide a reasonable prediction for the NNLO cross section. Our default choice to use $r_{\text {cut }} \geq 0.15 \%$ to obtain the $r_{\text {cut }} \rightarrow 0$ extrapolation appears to be a sound compromise between a sufficiently low $r_{\text {cut }}$ value and a good numerical convergence. The blue band constitutes a reasonable estimate of the remaining extrapolation uncertainty of our reference result. Since no cuts have been applied, our results can be compared with available analytic computations of the inclusive Higgs and $Z$ production cross sections. Such results are obtained with SUSHI [110] for Higgs boson production, and with ZWPROD [2,3] for Z-boson production, and are reported in Fig. 2 by the black solid lines with grey numerical error bands. The agreement is excellent and confirms that with MATRIX we control these computations at the subpermille level.

The next process we consider is $p p \rightarrow e^{+} v_{e}$ via an offshell $W^{+}$boson (third plot in Fig. 2). The $r_{\text {cut }}$ dependence is similar to the case of on-shell Higgs and $Z$ production with all fixed- $r_{\text {cut }}$ results deviating not more than about one permille from the extrapolated result. We therefore conclude that the stability fully justifies our reference prediction and that its blue uncertainty band, which is slightly larger than for the on-shell processes discussed before, gives a reasonable estimate of the uncertainty due to the $r_{\text {cut }}$ dependence of the cross 

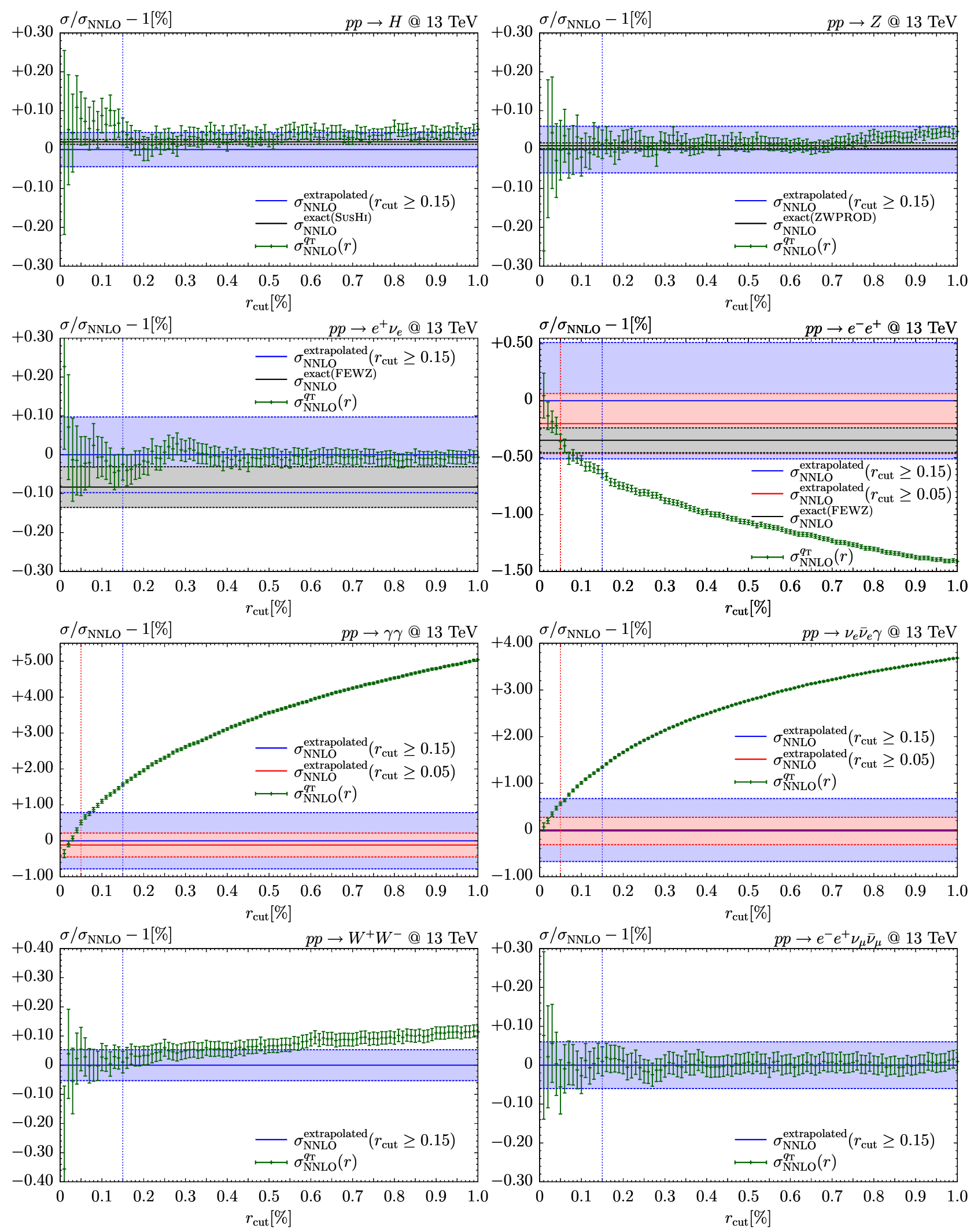

Fig. 2 Dependence of the NNLO cross sections on $r_{\text {cut }}$ for various processes. The NNLO results at fixed values of $r_{\text {cut }}$ are normalized to the $r_{\text {cut }} \rightarrow 0$ extrapolation obtained by using $r_{\text {cut }} \geq 0.15 \%$. The blue band represents the combined numerical and extrapolation uncertainty.

For processes with a large $r_{\text {cut }}$ dependence, the extrapolated result and uncertainty obtained by using $r_{\text {cut }} \geq 0.05 \%$ is shown in red. Where available, $r_{\text {cut }}$-independent reference results are black 
section. In the same plot we also report the result obtained with FEWZ [53], depicted by a solid black line with grey error bands. The MATRIX and FEWZ results are fully consistent within the respective numerical uncertainties. A similar level of agreement is obtained with the program NNLOJET [119] ${ }^{49}$ Since both FEWZ and NNLOJET are based on fully independent subtraction schemes, the above agreement can be considered as an important mutual consistency check of the three NNLO calculations.

Next, we discuss the dilepton production process $p p \rightarrow$ $e^{-} e^{+}$, which, in our default setup, exhibits a rather large $r_{\text {cut }}$ dependence. This is illustrated in the fourth plot of Fig. 2. As discussed in Sect. 6, the strong $r_{\text {cut }}$ dependence can be traced back to the presence of perturbative instabilities [111] affecting the fixed-order computation in the case of symmetric $p_{T}$ cuts. We have explicitly tested that if we change our default setup from $p_{T, \ell}>25 \mathrm{GeV}$ to $p_{T, e^{-}}>25 \mathrm{GeV}$ and $p_{T, e^{+}}>24 \mathrm{GeV}$, we get a stable $r_{\text {cut }}$ dependence. If we change this setup by letting the cut on $p_{T, e^{+}}$approach $25 \mathrm{GeV}$, the $r_{\text {cut }}$ dependence of the cross section becomes increasingly stronger. We also point out that, if we remove the lepton $p_{T}$ thresholds completely or choose them as small as $p_{T, \ell}>5 \mathrm{GeV}$, we obtain a flat $r_{\text {cut }}$ dependence of the cross section. The sizeable $r_{\text {cut }}$ dependence implies a larger uncertainty in the $r_{\text {cut }} \rightarrow 0$ extrapolation. We indeed see that by using the default setup the estimated uncertainty, represented by the blue band, is about $\pm 0.5 \%$. By reducing the minimum $r_{\text {cut }}$ value to $0.05 \%$, we reduce the extrapolation uncertainty by about a factor of 2 (red band) and we obtain a fully consistent result with the one obtained from $r_{\text {cut }} \geq 0.15 \%$. This is a strong indication that the extrapolation procedure is robust and provides a reasonable estimate of the ensuing uncertainty. In the same plot we report the result obtained with FEWZ: the agreement with the extrapolated results is excellent. A similar level of agreement is obtained with NNLOJET. As in the previous cases, the agreement of NNLO predictions obtained with fully independent methods confirms the robustness of the results that can be obtained with MATRIX. We remark that this conclusion holds also in a case, like the one of dilepton production with symmetric cuts, in which a fixed-order computation is challenged.

In terms of the $r_{\text {cut }}$ dependence of their cross sections, processes with an isolated photon in the final state suffer from large power-suppressed corrections. Since the case of $W \gamma$ production was discussed in Ref. [31], here we consider diphoton and $Z \gamma$ production with the $Z$ boson decaying to a neutrino pair. The corresponding results are shown in the fifth

\footnotetext{
$\overline{49}$ Note that we have set the CKM matrix to unity here, see Sect. 5.1.2, in order to be able to compare against the results of NNLOJET. The CKM input does not have any impact on the $r_{\text {cut }}$ dependence beyond statistical uncertainties, which is why the discussion above is valid irrespective of the chosen CKM settings.
}

and sixth plot in Fig. 2, respectively. Looking at our extrapolated reference results for $r_{\text {cut }} \geq 0.15 \%$ and $r_{\text {cut }} \geq 0.05 \%$, we see that they are nicely consistent with the behaviour of the numerical results below the respective minimal $r_{\text {cut }}$ value and that they are in neat mutual agreement. In particular, the result at the lowest $r_{\text {cut }}$ value $r_{\text {cut }}=0.01 \%$ is consistent with both the red and blue bands in all cases.

It is clear that, for dilepton, diphoton and $Z \gamma$ production, the extrapolation $r_{\text {cut }} \rightarrow 0$ of runs with $r_{\text {cut }} \geq 0.15 \%$ allows us to control the uncertainty of our NNLO predictions at the $0.5-1 \%$ level. If the minimal $r_{\text {cut }}$ value is decreased to $r_{\text {cut }}^{\min }=$ $0.05 \%$, the ensuing uncertainty is reduced to few permille. We have explicitly checked that this picture is common to all processes involving photons. We conclude that, taking into account the estimated $r_{\text {cut }}$ uncertainties, we obtain consistent predictions for all these processes.

The last two plots in Fig. 2 are representative $r_{\text {cut }}{ }^{-}$ dependence plots for on- and off-shell diboson production: The first one shows on-shell $W^{+} W^{-}$production, and the second one off-shell $Z Z$ production in the $2 \ell 2 v^{\prime}$ decay channel. These plots feature to a large extent the same behaviour as observed for on-shell Higgs and vector-boson production processes: In general, the NNLO cross section is very stable with respect to $r_{\text {cut }}$ over two orders of magnitude. The $r_{\text {cut }}$ dependence of the $W^{+} W^{-}$cross section seems to have a very slight slope, which, however, is perfectly modelled by the extrapolation. Our default choice of the minimal $r_{\text {cut }}$ value leads to a reasonable reference result with the estimated uncertainties being meaningful and in some cases even a bit conservative.

In summary, for most of the processes implemented in the first release of MATRIX, NNLO predictions can generally be controlled at the one permille level (or better). For processes with a large $r_{\text {cut }}$ dependence like those involving photons or Drell-Yan dilepton pairs, fiducial cross sections can be computed with uncertainties of few permille by using the setting switch_qT_accuracy $=1$. This accuracy should be sufficient for all practical purposes.

\section{Summary}

In this paper we have introduced the new computational framework MATRIX [1], which allows a user to produce NNLO QCD predictions for a wide class of hadron-collider processes. Using the $q_{T}$-subtraction formalism, our computations are fully differential in the phase space of the finalstate particles and of the associated QCD radiation, thereby enabling the evaluation of arbitrary IR safe observables. Since our implementation is completely general, it is applicable to the computation of NNLO corrections to any process with colourless final states. The list of available processes is therefore limited only by the availability of two-loop ampli- 
tudes for the Born-level processes. The first MATRIX release involves $2 \rightarrow 1$ and $2 \rightarrow 2$ hadronic reactions with Higgs and vector bosons in the final state. In particular, we consider final states with two, three and four leptons (plus missing energy) from the decays of the vector bosons, and we account for all resonant and non-resonant diagrams with offshell effects and spin correlations. This enables the evaluation of cross sections with realistic cuts to define any fiducial volume.

We have presented a detailed description of the first release of the MATRIX code. Besides the theoretical framework and the tools MATRIX is based on, we have focused on the technical aspects relevant for a user to produce fully differential NNLO results. MATRIX features automatic compilation and running through dedicated scripts. The most popular clusters are preconfigured and can be simply selected by the user. Having specified a target precision and a desired runtime per job, the code automatically determines the required parallelization in each run. In combination with the fast numerical multi-channel integration offered by MUNICH, this allows us to obtain accurate NNLO results even for the most complicated of the available processes on a middle-sized cluster in less than a couple of days, simpler processes being significantly faster. With every run MATRIX provides not only the central prediction, but automatically evaluates the effect of independent factorization- and renormalization-scale variations in order to obtain an estimate of the perturbative uncertainties at each order. Furthermore, by simultaneously computing NNLO cross sections at several values of the $q_{T^{-}}$ subtraction parameter $r_{\text {cut }}$, MATRIX performs an extrapolation $r_{\text {cut }} \rightarrow 0$ of the integrated cross section in order to provide its final prediction that includes an extrapolation uncertainty. Such procedure allows us to offer a robust estimate of the systematic uncertainty due to the $q_{T}$-subtraction procedure. Both scale variations and the $r_{\text {cut }}$ extrapolation procedure are fully automated within MATRIX without the need of extra computing power.

In particular for processes with a large $r_{\text {cut }}$ dependence, such as dilepton production or processes with isolated photons, MATRIX is able to significantly improve over predictions computed at fixed $r_{\text {cut }}$ values by performing the $r_{\text {cut }} \rightarrow 0$ extrapolation. Besides an improved accuracy in the central prediction, our procedure includes a conservative estimate of systematic uncertainties, which allows the user to control the precision of these processes at the level of few permille, when using corresponding settings.

We have discussed in detail all relevant MATRIX input cards accessible to the user. Besides standard settings applicable to all processes, information specific to each individual process has been provided, such as suitable dynamic scales which are predefined for certain processes and processspecific cuts. The latter facilitate the restriction of the phase space to fiducial volumes as defined by the LHC experiments.
In combination with a general way to define distributions, this enables the possibility to compute fiducial cross sections and distributions that can be directly compared to unfolded experimental data.

Reference predictions for the integrated cross sections of all processes available in MATRIX have been provided at LO, NLO and NNLO in the default setups. For the NNLO cross section we have quoted predictions for a fixed value $r_{\text {cut }}=0.15 \%$ and the final NNLO result after performing the extrapolation $r_{\text {cut }} \rightarrow 0$. We have studied the impact of radiative corrections for each of these processes as well as the impact of the loop-induced $g g$ component, where applicable. The impact of NLO and NNLO QCD corrections is generally large. While NLO corrections typically range between 30 and $100 \%$, NNLO corrections are still as large as 3-30\% for the processes and scenarios we have considered. The size of radiative corrections is typically widely un-affected if only fiducial cuts for particle identification, like transversemomentum thresholds, geometric (pseudo-)rapidity ranges or isolation cuts are applied (see, e.g., inclusive on-shell $Z Z$ production and off-shell $Z Z \rightarrow 4 \ell$ production in a $Z Z$ signal region). This is in general no longer true if the considered cuts modify the dominant resonance structures (e.g. $Z Z \rightarrow 4 \ell$ production in the $H \rightarrow Z Z$ background region). If real radiation is restricted, in particular by a veto against jets, the size of higher-order corrections is strongly suppressed, and NLO and NNLO $K$ factors can be very different as compared to the inclusive phase-space (see, e.g., inclusive on-shell $W^{+} W^{-}$ production and off-shell $W W \rightarrow 2 \ell 2 v$ production, which requires a jet veto to suppress the top-quark backgrounds).

Scale variations tend to underestimate the uncertainty from missing higher-order contributions at LO, and also widely at NLO. This is due to the fact that vector-boson and vector-boson pair production processes are driven by $q \bar{q}$ initial states at LO ( $g g$ initial states in case of Higgs boson production). The $g q$ channel opens up only at NLO, and NNLO is the first order where all partonic channels are contributing. As a consequence, in most of the cases NNLO uncertainties obtained through scale variations should provide the correct order of magnitude of yet un-calculated perturbative QCD contributions. When NNLO corrections are particularly large, as in the case of diphoton production, a more conservative estimate of missing higher-order contributions can be obtained by considering the difference with the previous order.

All the vector-boson pair production processes with an electrically neutral final state include a loop-induced $g g$ contribution at NNLO. Its size strongly depends on the considered process and can range between roughly $10 \%$ and $60 \%$ of the NNLO corrections. In cases where radiative corrections are suppressed by a jet veto, since the $g g$ component is not affected due to its Born-level kinematics, it may provide the dominant NNLO contribution. As the $g g$ component is 
effectively only $\mathrm{LO}$ accurate, scale variations might underestimate the actual size of its missing perturbative corrections in some cases.

To conclude, we believe that the numerical tool presented in this paper will be highly valuable for the high-energy community. Several experimental studies have already used the results obtained with MATRIX for data-theory comparison in SM measurements (see, e.g., Refs. [107,120-138]) and for background estimates in various new-physics searches. MATRIX [1] can be used to produce benchmark predictions for a wide range of processes relevant both for SM measurements and as backgrounds to Higgs and new-physics searches. Extensions of the code to include additional processes, the inclusion of further perturbative contributions (e.g. NLO corrections to the $g g$ channel or EW corrections) as well as the resummation of certain classes of logarithmic contributions are left for future work.

Acknowledgements We are grateful to Dirk Rathlev for his contributions at the beginning of the MATRIX project. We would like to thank Stefano Catani for comments on the manuscript and Alexander Huss for providing cross checks for the Drell-Yan processes with NNLOJET. We are indebted to Jonas Lindert, Philipp Maierhöfer and Stefano Pozzorini for their continuous support on OPENLOOPS. We thank Thomas Gehrmann, Andreas von Manteuffel and Lorenzo Tancredi for providing us with private implementations of the two-loop amplitudes to on-shell $W^{+} W^{-}$and $Z Z$ production. This research was supported in part by the Munich Institute for Astro- and Particle Physics (MIAPP) of the DFG cluster of excellence "Origin and Structure of the Universe", by the Swiss National Science Foundation (SNF) under contracts CRSII2141847, 200020-169041 and by the Research Executive Agency (REA) of the European Union under the Grant Agreement number PITN-GA2012-316704 (HiggsTools). The work of MW is supported by the ERC Consolidator Grant 614577 HICCUP.

Open Access This article is distributed under the terms of the Creative Commons Attribution 4.0 International License (http://creativecomm ons.org/licenses/by/4.0/), which permits unrestricted use, distribution, and reproduction in any medium, provided you give appropriate credit to the original author(s) and the source, provide a link to the Creative Commons license, and indicate if changes were made.

Funded by SCOAP ${ }^{3}$.

\section{Appendix A Predefined cuts}

In this Appendix the process-dependent cuts introduced in Sect. 5.2 are explained in more detail. It can be used as a dictionary since the respective cuts in Sect. 5.2 contain interactive links to their explanations in Table 12.

\section{Appendix B Modifications of the C++ code (advanced user)}

The user is generally advised to contact the authors if he/she is interested in changing any parts of the $\mathrm{C}++$ code in order to define new dynamic scales, fiducial cuts or distributions. Below we provide some general guidance on how such implementations can be realized in the $\mathrm{C}++$ code. Any changes of the $\mathrm{C}++$ code require recompilation of the relevant process. This can be done with the matrix script, but in certain cases it might be simpler to use directly the Makefile the matrix script has created during the initial compilation of a process with ID $\$\{$ process_id\}, by typing

\$ make \$\{process_id\}

This enables recompilation without cleaning the whole process and without checking again whether all the relevant libraries are correctly installed.

\section{B.1 General C++ commands}

\section{B.1.1 Access to particle groups}

In the $\mathrm{C}++$ routines for the user-defined scales and cuts one has access to all particle groups listed in Fig. 3 and the ones defined by the user, see below. As discussed before, the particle groups are ordered in the transverse momentum of the particles and can be accessed via PARTICLE ( "\$ \{particle_group\} ") [index], where $\$\{$ particle_group\} is one of the particle groups defined in Table 3 or the user-defined ones, and index indicates the position in the $p_{T}$-ordering of the group starting from the hardest one at index $=0$.

For example, the hardest lepton can be accessed via particle hardest_lepton = PARTICLE ("lep")[0];

The particle class itself has various predefined class variables that can be directly used. For example, the $p_{T}$ or squared invariant mass can be determined as follows:

double pT_hardest_lepton = PARTICLE ("lep") [0].pT; double m2_hardest_lepton = PARTICLE ("lep") [0].m2;

These can also determined via the full four-vector that has certain predefined functions and can be used as follows:

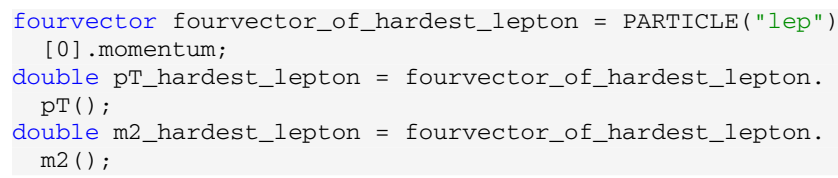

or similar functions for other observables, such as rapidity and pseudo-rapidity

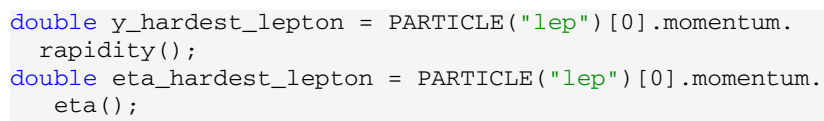


Table 12 Explanations of the pre-defined cuts available in the various MATRIX processes. See Sect. 5.2 for which cuts belong to which process

\begin{tabular}{|c|c|}
\hline $\begin{array}{l}\text { user_switch } \\
\text { user_cut }\end{array}$ & Description \\
\hline lepton_identification & $\begin{array}{l}\text { Switch to change between different identification procedures of } Z \text { and } W \text { bosons in the } \\
\text { same-flavour channels of } Z Z \text { and } W Z \text { production. See Sects. 5.2.4.4 and 5.2.4.9 for details. }\end{array}$ \\
\hline M_leplep & Switch for cuts on invariant mass $m_{\ell \ell}$ of all possible lepton pairs \\
\hline min_M_leplep & Minimal requirement $m_{\ell \ell}>$ min_M_leplep for all lepton pairs \\
\hline max_M_leplep & Maximal requirement $m_{\ell \ell}<\max \_\mathrm{M} \_l e p l e p$ for all lepton pairs \\
\hline M_leplep_osSF & Switch for cuts on invariant mass $m_{\ell^{-} \ell^{+}}$of opposite-sign, same-flavour (OSSF) lepton pairs \\
\hline min_M_leplep_OSSF & Minimal requirement $m_{\ell^{-} \ell^{+}}>$min_M_leplep_OSSF for OSSF pairs \\
\hline max_M_leplep_osSF & Maximal requirement $m_{\ell^{-} \ell^{+}}<$max_M_leplep_OSSF for OSSF pairs \\
\hline min_M_Z1_OSSF & Minimal requirement $m_{\ell^{-} \ell^{+}}>$min_M_Z1_OSSF for OSSF pair closer to $m_{Z}$ \\
\hline max_M_Z1_oSSF & Maximal requirement $m_{\ell^{-} \ell^{+}}<$max_M_Z1_OSSF for OSSF pair closer to $m_{Z}$ \\
\hline M_Zrec & Switch for cuts on invariant mass $m_{\ell^{-} \ell^{+}}$of lepton pairs associated with $Z$ bosons \\
\hline min_M_Zrec & Minimal requirement $m_{\ell^{-} \ell^{+}}>$min_M_Zrec for (reconstructed) $Z$ bosons \\
\hline max_M_Zrec & Maximal requirement $m_{\ell^{-} \ell^{+}}<$max_M_Zrec for (reconstructed) $Z$ bosons \\
\hline delta_M_Zrec_MZ & $\begin{array}{l}\text { Switch for cuts on absolute difference between invariant mass } m_{\ell^{-} \ell^{+}} \text {of lepton pairs associated } \\
\text { with } Z \text { bosons and the } Z \text {-boson mass }\end{array}$ \\
\hline max_delta_M_Zrec_MZ & Maximal requirement $\left|m_{\ell^{-} \ell^{+}}-m_{Z}\right|<$ max_delta_M_Zrec_MZ for (reconstructed) $Z$ bosons \\
\hline delta_M_lepleplep_MZ & $\begin{array}{l}\text { Switch for cuts on absolute difference between invariant mass } m_{\ell \ell \ell} \text { of 3-lepton system and the } \\
Z \text {-boson mass }\end{array}$ \\
\hline min_delta_M_lepleplep_Mz & Minimal requirement $\left|m_{\ell \ell \ell}-m_{Z}\right|>$ min_delta_M_lepleplep_MZ \\
\hline M_4lep & Switch for cuts on invariant mass $m_{\ell \ell \ell \ell}$ of 4-lepton system \\
\hline min_M_4lep & Minimal requirement $m_{\ell \ell \ell \ell}>$ min_M_4lep for 4-lepton system \\
\hline max_M_4lep & Maximal requirement $m_{\ell \ell \ell \ell}<$ max_M_4lep for 4-lepton system \\
\hline min_delta_M_4lep & Minimal requirement $\left|m_{\ell \ell \ell \ell}-m_{Z}\right|>$ min_delta_M_4lep for 4-lepton system \\
\hline max_delta_M_4lep & Maximal requirement $\left|m_{\ell \ell \ell \ell}-m_{Z}\right|<$ max_delta_M_4lep for 4-lepton system \\
\hline M_leplepnunu & Switch for cuts on invariant mass $m_{\ell \ell v v}$ of 2-lepton-2-neutrino system \\
\hline min_M_leplepnunu & Minimal requirement $m_{\ell \ell v v}>$ min_delta_M_leplepnunu for $2 \ell 2 v$ system \\
\hline max_M_leplepnunu & Maximal requirement $m_{\ell \ell v v}<$ max_delta_M_leplepnunu for $2 \ell 2 v$ system \\
\hline min_delta_M_leplepnunu & Min. requirement $\left|m_{\ell \ell v v}-m_{Z}\right|>$ min_delta_M_leplepnunu for $2 \ell 2 v$ system \\
\hline max_delta_M_leplepnunu & Max. requirement $\left|m_{\ell \ell v v}-m_{Z}\right|<$ max_delta_M_leplepnunu for $2 \ell 2 v$ system \\
\hline pT_leplep & Switch for cuts on transverse momentum of lepton pair $p_{T, \ell \ell}$ \\
\hline min_pT_leplep & Minimal requirement $p_{T, \ell \ell}>$ min_pT_leplep for lepton pair \\
\hline pT_lep_1st & Switch for cuts on transverse momentum of hardest lepton $p_{T, \ell_{1}}$ \\
\hline min_pT_lep_1st & Minimal requirement $p_{T, \ell_{1}}>$ min_pT_lep_1st for hardest lepton \\
\hline pT_lep_2nd & Switch for cuts on transverse momentum of second-hardest lepton $p_{T, \ell_{2}}$ \\
\hline min_pT_lep_2nd & Minimal requirement $p_{T, \ell_{2}}>$ min_pT_lep_2nd for second-hardest lepton \\
\hline
\end{tabular}


Table 12 continued

\begin{tabular}{|c|c|}
\hline $\begin{array}{c}\text { user_switch } \\
\text { user_cut }\end{array}$ & Description \\
\hline lepton_cuts & Switch for cuts on transverse momentum of (second-)hardest lepton $p_{T, \ell_{1}}\left(p_{T, \ell_{2}}\right)$ \\
\hline min_pT_lep_1st & Minimal requirement $p_{T, \ell_{1}}>$ min_pT_lep_1st for hardest lepton \\
\hline min_pT_lep_2nd & Minimal requirement $p_{T, \ell_{2}}>$ min_pT_lep_2nd for second-hardest lepton \\
\hline leading_lepton_cuts & Switch for flavour-dependent cuts on hardest-lepton transverse momentum $p_{T, \ell_{1}}$ \\
\hline min_pT_1st_if_e & Minimal requirement $p_{T, \ell_{1}}>$ min_pT_1st_if_e if hardest lepton is electron \\
\hline min_pT_1st_if_mu & Minimal requirement $p_{T, \ell_{1}}>$ min_pT_1st_if_mu if hardest lepton is muon \\
\hline lepz_cuts & $\begin{array}{l}\text { Switch for cuts on transverse momentum of (second-)hardest lepton associated with } Z \text {-boson } \\
\text { decay } p_{T, \ell_{Z, 1}}\left(p_{T, \ell_{Z, 2}}\right)\end{array}$ \\
\hline min_pT_lepz_1st & Minimal requirement $p_{T, \ell_{Z, 1}}>$ min_pT_lepz_1st for hardest lepton of $Z$ \\
\hline min_pT_1epz_2nd & Minimal requirement $p_{T, \ell_{Z, 2}}>$ min_pT_lepZ_2nd for second-hardest lepton of $Z$ \\
\hline lepW_cuts & Switch for cuts on lepton associated with $W$-boson decay \\
\hline min_pT_lepW & Minimal requirement $p_{T, \ell_{W}}>$ min_pT_lepW for lepton from $W$-boson decay \\
\hline max_eta_lepw & Minimal requirement $\eta_{\ell_{W}}<$ max_eta_lepW for lepton from $W$-boson decay \\
\hline R_leplep & Switch for cuts on lepton separation in $\Delta R_{\ell \ell}=\sqrt{\Delta y_{\ell \ell}^{2}+\Delta \phi_{\ell \ell}^{2}}$ \\
\hline min_R_leplep & Minimal requirement $\Delta R_{\ell \ell}>$ min_R_leplep for all lepton pairs \\
\hline R_lepzlepz & $\begin{array}{l}\text { Switch for cuts on separation between leptons associated with } Z \text {-boson decay in } \\
\qquad \Delta R_{\ell_{Z} \ell_{Z}}=\sqrt{\Delta y_{\ell_{Z} \ell_{Z}}^{2}+\Delta \phi_{\ell_{Z} \ell_{Z}}^{2}}\end{array}$ \\
\hline min_R_lepzlepz & Minimal requirement $\Delta R_{\ell_{Z} \ell_{Z}}>$ min_R_lepZlepZ for leptons of $Z$ decay \\
\hline R_lepzlepw & $\begin{array}{l}\text { Switch for cuts on separation between leptons associated with } Z \text {-boson decay and lepton } \\
\text { associated with } W \text {-boson decay in } \Delta R_{\ell_{Z} \ell_{W}}=\sqrt{\Delta y_{\ell_{Z} \ell_{W}}^{2}+\Delta \phi_{\ell_{Z} \ell_{W}}^{2}}\end{array}$ \\
\hline min_R_lepzlepw & Minimal requirement $\Delta R_{\ell_{Z} \ell_{W}}>$ min_R_lepZlepW for leptons of $Z$ and $W$ decay \\
\hline phi_leplep & Switch for cuts on azimuthal separation $\Delta \phi_{\ell \ell}$ between all lepton pairs \\
\hline min_phi_leplep & Minimal requirement $\Delta \phi_{\ell \ell}>$ min_phi_leplep for all lepton pairs \\
\hline phi_leplep_nunu & $\begin{array}{l}\text { Switch for cuts on azimuthal separation } \Delta \phi_{\ell \ell, v v} \text { between the transverse-momentum vectors of the } \\
\text { 2-lepton system } \boldsymbol{p}_{\boldsymbol{T}, \ell \ell} \text { and the missing energy } \mathbf{p}_{\mathbf{T}}^{\text {miss }}\end{array}$ \\
\hline min_phi_leplep_nunu & Minimal requirement $\Delta \phi_{\ell \ell, v v}>$ min_phi_leplep_nunu \\
\hline electron_cuts & Switch for cuts on transverse momentum of (second-)hardest electron $p_{T, e_{1}}\left(p_{T, e_{2}}\right)$ \\
\hline min_pT_e_1st & Minimal requirement $p_{T, e_{1}}>$ min_pT_e_1st for hardest electron \\
\hline min_pT_e_2nd & Minimal requirement $p_{T, e_{2}}>$ min_pT_e_2nd for second-hardest electron \\
\hline muon_cuts & Switch for cuts on transverse momentum of (second-)hardest muon $p_{T, \mu_{1}}\left(p_{T, \mu_{2}}\right)$ \\
\hline min_pT_mu_1st & Minimal requirement $p_{T, \mu_{1}}>$ min_pT_mu_1st for hardest muon \\
\hline min_pT_mu_2nd & Minimal requirement $p_{T, \mu_{2}}>$ min_pT_mu_2nd for second-hardest muon \\
\hline gap_eta_e & Switch for detector gap in absolute pseudo-rapidity $\left|\eta_{e}\right|$ of electrons \\
\hline
\end{tabular}


Table 12 continued

\begin{tabular}{|c|c|}
\hline $\begin{array}{l}\text { user_switch } \\
\text { user_cut }\end{array}$ & Description \\
\hline gap_min_eta_e & Start of the gap, keeping only events with $\left|\eta_{e}\right|<$ gap_min_eta_e \\
\hline gap_max_eta_e & End of the gap, keeping only events with $\left|\eta_{e}\right|>$ gap_max_eta_e \\
\hline M_gamgam & Switch for cuts on invariant mass $m_{\gamma \gamma}$ of photon pairs \\
\hline min_M_gamgam & Minimal requirement $m_{\gamma \gamma}>$ min_M_gamgam for photon pairs \\
\hline max_M_gamgam & Maximal requirement $m_{\gamma \gamma}<$ min_M_gamgam for photon pairs \\
\hline pT_gam_1st & Switch for cuts on transverse momentum of hardest photon $p_{T, \gamma_{1}}$ \\
\hline min_pT_gam_1st & Minimal requirement $p_{T, \gamma_{1}}>$ min_pT_gam_1st for hardest photon \\
\hline R_gamgam & Switch for cuts on photon separation in $\Delta R_{\gamma \gamma}=\sqrt{\Delta y_{\gamma \gamma}^{2}+\Delta \phi_{\gamma \gamma}^{2}}$ \\
\hline min_R_gamgam & Minimal requirement $\Delta R_{\gamma \gamma}>$ min_R_gamgam for photon pairs \\
\hline gap_eta_gam & Switch for detector gap in absolute pseudo-rapidity $\left|\eta_{\gamma}\right|$ of photons \\
\hline gap_min_eta_gam & Start of the gap, keeping only events with $\left|\eta_{\gamma}\right|<$ gap_min_eta_gam \\
\hline gap_max_eta_gam & End of the gap, keeping only events with $\left|\eta_{\gamma}\right|>$ gap_max_eta_gam \\
\hline M_lepgam & Switch for cuts on invariant mass $m_{\ell \gamma}$ of lepton-photon pairs \\
\hline min_M_lepgam & Minimal requirement $m_{\ell \gamma}>$ min_M_lepgam for lepton-photon pairs \\
\hline R_lepgam & Switch for cuts on lepton-photon separation in $\Delta R_{\ell \gamma}=\sqrt{\Delta y_{\ell \gamma}^{2}+\Delta \phi_{\ell \gamma}^{2}}$ \\
\hline min_R_lepgam & Minimal requirement $\Delta R_{\ell \gamma}>$ min_R_lepgam for lepton-photon pairs \\
\hline R_lepjet & Switch for cuts on lepton-jet separation in $\Delta R_{\ell j}=\sqrt{\Delta y_{\ell j}^{2}+\Delta \phi_{\ell j}^{2}}$ \\
\hline min_R_lepjet & Minimal requirement $\Delta R_{\ell j}>$ min_R_lepjet for lepton-jet pairs \\
\hline lep_iso & $\begin{array}{l}\text { Switch for special isolation of lepton-lepton and lepton-parton pairs, as used for example in the } \\
Z Z \text { Higgs background, see Ref. [139]. For each lepton } i \text { we compute the sum of the transverse } \\
\text { momenta over all leptons and partons in a certain } R \text { cone around } i \text { and take the ratio to its } \\
\text { transverse momentum. All events are discarded where this ratio is below a certain threshold: }\end{array}$ \\
\hline & $\sum_{\substack{j \in\{\ell, \text { partons }\} \\
\text { with } \Delta R_{i j}<\delta_{0}}} p_{T, j} / p_{T, i}<\epsilon$ \\
\hline lep_iso_delta_0 & $\delta_{0}$ in the formula above \\
\hline lep_iso_epsilon & $\epsilon$ in the formula above \\
\hline R_ejet & Switch for cuts on electron-jet separation in $\Delta R_{e j}=\sqrt{\Delta y_{e j}^{2}+\Delta \phi_{e j}^{2}}$ \\
\hline min_R_ejet & Minimal requirement $\Delta R_{e j}>$ min_R_ejet for electron-jet pairs \\
\hline R_gamjet & Switch for cuts on photon-jet separation in $\Delta R_{\gamma j}=\sqrt{\Delta y_{\ell j}^{2}+\Delta \phi_{\ell j}^{2}}$ \\
\hline min_R_gamjet & Minimal requirement $\Delta R_{\gamma j}>$ min_R_gamjet for photon-jet pairs \\
\hline mT_CMS & Switch for cuts on transverse mass $m_{T, W}$ of $W$ boson as defined by CMS [140] \\
\hline min_mT_CMS & Minimal requirement $m_{T, W}>$ min_mT_CMS for the $W$ boson \\
\hline
\end{tabular}


Table 12 continued

\begin{tabular}{|c|c|}
\hline $\begin{array}{c}\text { user_switch } \\
\text { user_cut }\end{array}$ & Description \\
\hline pT_W & Switch for cuts on transverse momentum $p_{T, \ell v_{\ell}}$ of (identified) $W$ bosons \\
\hline min_pT_W & Minimal requirement $p_{T, \ell v_{\ell}}>$ min_pT_W for (identified) $W$ bosons \\
\hline max_pT_W & Maximal requirement $p_{T, \ell \nu_{\ell}}<$ max_pT_W for (identified) $W$ bosons \\
\hline MT_Wrec & Switch for cuts on transverse mass $m_{T, \ell v_{\ell}}$ of (identified) $W$ bosons, defined by \\
\hline & $m_{T, \ell v_{\ell}}=\sqrt{\left(E_{T, \ell}+E_{T, \nu_{\ell}}\right)^{2}-\left(p_{T, \ell \nu_{\ell}}\right)^{2}}$ with $\left(E_{T, x}\right)^{2}=m_{x}^{2}+\left(p_{T, x}\right)^{2}$ \\
\hline min_MT_Wrec & Minimal requirement $m_{T}^{\ell v_{\ell}}>$ min_mT_Wrec for (identified) $W$ bosons \\
\hline rel_pT_miss & $\begin{array}{l}\text { Switch for cuts on the relative missing transverse momentum } p_{T}^{\text {miss,rel }} \text {, which is defined as } \\
p_{T}^{\text {miss }} \times \sin |\Delta \phi| \text {, where } \Delta \phi \text { is the azimuthal separation between } \mathbf{p}_{\mathbf{T}}^{\text {miss }} \text { and the momentum of the } \\
\text { closest lepton, see Ref. [130] }\end{array}$ \\
\hline min_rel_pT_miss & Minimal requirement $p_{T}^{\text {miss,rel }}>$ min_rel_pT_miss \\
\hline
\end{tabular}

It is important to note that the four-vectors of the particles can be simply added to define a new four-vector, where the same functions can be used. For example, the transverse mass $\left(\sqrt{m^{2}+p_{T}^{2}}\right)$ of the system of the lepton pair (hardest and second-hardest lepton) can be simply computed by

fourvector fourvector_of_leplep = PARTICLE("lep") [0] .

momentum+PARTICLE ( "lep") [1] .momentum;

double mT_leplep = fourvector_of_leplep.ET();

Similarly, one may also directly sum two objects of type particle to define a new particle whose momentum corresponds to the sum. Thus, it would be equivalent to compute the transverse mass of the system of the lepton pair by using

particle leplep = PARTICLE("lep")[0] + PARTICLE("lep")[1] double mT_leplep = leplep.ET;

Finally, if a certain observable one may want to compute is neither predefined in the particle nor the fourvector class, one can always access the momenta directly by using

fourvector fourvector_of_hardest_lep = PARTICLE("lep") [0]. momentum;

double $\mathrm{E}$ hardest lep = fourvector of hardest_lepton.x0() ;

double $\mathrm{x}$ hardest lep $=$ fourvector of hardest lepton $\mathrm{x} 1()$;

double y_hardest_lep = fourvector_of_hardest_lepton.x2 ();

double z_hardest_lep = fourvector_of_hardest_lepton.x3 ();

and compute the desired observable from the explicit momentum components.

\section{B.1.2 Access to user-defined parameters}

In the file parameter. dat three types of user-defined parameters can be added, which are available in the $\mathrm{C}++$ code and can be directly accessed throughout the processspecific $\mathrm{C}++$ files inside the folder MATRIX_v1.0.0 /prc/\$ \{process_id\}/user.
- An integer-valued user parameter is added via

user_switch my_integer_parameter $=1$

to the file parameter. dat and is accessed in the $\mathrm{C}++$ code by

static int my_integer

= USERSWITCH ("my_integer_parameter");

Such switches are useful in many respects, two already used examples are to turn on and off cuts, or to choose between different identification procedures.

- A real-valued user parameter is added via

user_cut my_real_parameter $=1.23$

to the file parameter. dat and is accessed in the $\mathrm{C}++$ code by

static double my_real = USERCUT("my_real_parameter") ;

Such real parameters are useful in many respects, the most important example is their use to define and

implement cuts that can be changed later from the file parameter. dat without recompilation of the code.

- Finally, a new particle (group) can be defined by adding

user_particle my_own_particle = my_own_particl

to the file parameter. dat. Only if defined this way, it can be filled in the $\mathrm{C}++$ code,

USERPARTICLE ( "my_own_particle"). push_back (PARTICLE("lep")[0]+ PARTICLE("lep")[1]) ; 
which would add a particle whose momentum is the sum of the hardest and second-hardest lepton to the userdefined particle group my_own_particle. If a userdefined particle group is filled with more than one particle, the usual $p_{T}$-ordering is done automatically before the respective particle group is used in scales, cuts or distributions. Note that a USERPARTICLE may only be filled in a certain position of the code, see below in Appendix B.2, and that it can be accessed later like all other particle groups via the container PARTICLE.

\section{B.2 Definition of a new particle group}

The predefined particle groups are sufficient for most practical cases. However, the user is allowed to define his own particle group by filling the respective four-vectors. This can be very useful if an intermediate particle cannot be unambiguously reconstructed, like in the case of the SF channel in $Z Z$ (or $W^{ \pm} Z$ ) production, where an identification procedure is needed for the $Z$ (and the $W$ ) bosons. As described in Sects. 5.2.4.4 and 5.2.4.9, such definition of process-specific particle groups is done intrinsically for these two processes following different identification procedures used by ATLAS and CMS. In the following we describe the necessary steps for a user to add his own definition of a particle group to any process.

The relevant $\mathrm{C}++$ file is MATRIX_v1.0.0/prc/ $\$\{$ process_id\}/user/specify.particles.cxx to fill user-defined particles. As described above, one has to add the definition of a new particle group to the file parameter. dat. After that the respective particle group exists as an empty array of objects of type particle inside the $\mathrm{C}++$ code, which must be filled by appending at least one object of type particle to the array.

Let us give a simple example: A new particle group should be filled depending on the value of an integer switch with either the hardest lepton or the second-hardest lepton. The relevant input in the file parameter. dat would look like

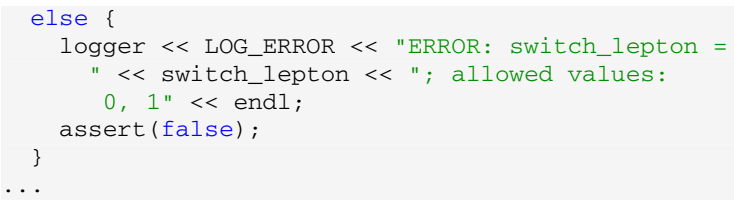

Bear in mind that after definition of a USERPARTICLE, the respective particle group is automatically filled afterwards. If the user-defined particle group contains more than one particle, the usual $p_{T}$-ordering is applied. The new particle group can then be accessed via the standard PARTICLE container, e.g.

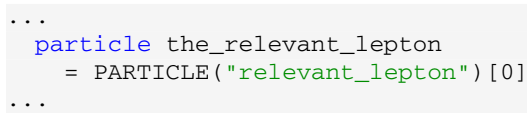

At this point the definition of the new particle has no practical effect yet, but one could now use the new particle group in the definition of a cut or for a distribution, and then decide via the switch in the input file whether it uses the hardest lepton or the second-hardest lepton. Such simple example may not appear to be extremely useful, however, this changes drastically if such cut or distribution is done according to a more complicated identification of a particle. In that case the identification procedure can be switched onthe-fly without the need of recompilation and without having to reimplement the same cuts and distributions for every new identification procedure. We refer the interested reader to MATRIX_V1.0.0/prc/ppeeexex04/user/

specify.particles.cxx for a sample implementation.

\section{B.3 Implementation of a new dynamic scale}

The relevant $\mathrm{C}++$ file is MATRIX_v1.0.0/prc/\$ \{process_id\}/user/specify.scales.cxx to add a new user-defined dynamic scale. All processes have at least two dynamic scales already implemented, and one can follow these implementations. In principle, one is free to code whatever one desires in that file, without taking care of the existing

user_particle relevant_lepton = relevant_lepton \# lepton, depending on switch_lepton

user_switch switch_lepton = 0 \# (0) hardest lepton, (1) second-hardest lepton

and the relevant $\mathrm{C}++$ code in the specify.particles . cxx would be

static int switch_lepton = USERSWITCH("switch_lepton");

if (switch_lepton $==0$ ) \{

USERPARTICLE ( "relevant_lepton" ).push_back (PARTICLE ("lep") [0]); \}

else if (switch_lepton $==1$ ) \{

USERPARTICLE ("relevant lepton").push back (PARTICLE ("lep") [1]); structure. One only has to make sure that in the end the variable temp_mu_central is set to the correct value. However, we recommend to follow the existing structure of the if and else if blocks to keep with the functionality of choosing different dynamic scales in the file parameter. dat.

Let us give a simple example, for completeness: If we want to add a dynamic scale 123 that computes the sum of the $Z$-boson mass and the transverse momentum of the hardest lepton, we would set 


dynamic_scale $=123 \#$ dynamic ren./fac. scale

in the file parameter. dat and add an else if block to the specify.scales.cxx file:

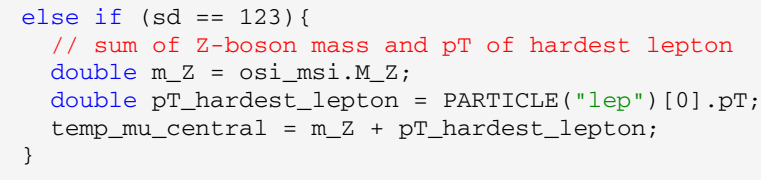

\section{B.4 Implementation of a new user-defined cut}

The relevant $\mathrm{C}++$ file is MATRIX_v1.0.0/prc/\$ \{process_id\}/user/specify.cuts.cxx to add a new user-defined cut. Most processes already have several process-specific cuts implemented, and one can in principle follow these implementations. Also in this file the user is essentially free to implement whatever he deserves without taking care of the existing structure. The only relevant information is that under whatever conditions one requires an event to be discarded, one sets

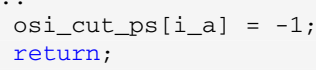

in order to cut the current phase-space point. The relevant momenta at each event are accessed via the particle groups as explained above. Nevertheless, we recommend to keep the existing structure by defining cuts via the user_switch and user_cut parameters that can be interactively changed afterwards in the file parameter. dat without recompilation of the $\mathrm{C}++$ code, instead of hard-coding such information in the file specify. cuts.cxx.

As a simple example we consider a lower cut on the absolute rapidity difference between the hardest and second-hardest lepton. Such cuts are added to the file parameter.dat,

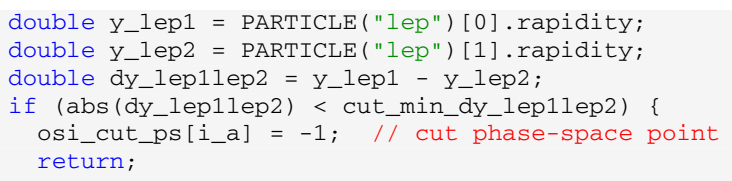

B.5 Implementation of a new observable for distributions

The relevant C++ file is MATRIX_v1.0.0/srC-MUNICH / classes/xdistribution. cpp to add a new userdefined distribution. Note that this part of the code is not specific to a certain process, and any observable implemented here can in principle be used in all processes. The relevant routine of the xdistribution class is

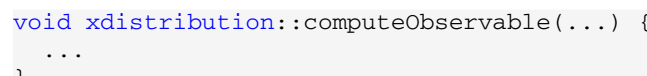

A rather comprehensive description of how to add a new distribution can be found commented inside this routine. We summarize the most important information. As pointed out in Sect. 5.1.3, each observable has a certain type identifier distributiontype set in the file distribution.dat, which must be specified in every distribution block. Inside the computeobservable routine of the xdistribution.cpp file, we can add a new distribution-type by extending the if and else if blocks for xdistribution_type, which corresponds to the string set for distributiontype in the file distribution. dat. The sum of the momenta of particle $i$ defined for each distribution in the file distribution. dat is saved to an array with entries of type fourvector called reconstructedParticles [i] inside the $\mathrm{C}++$ code. The distributions can now be defined using these particles, by setting the variable observable to the value of the observable that should be binned for the desired xdistribution_type.

Let us consider a simple example where we want to plot the distribution of events in the sum of the rapidities of the two and implemented into the $\mathrm{C}++$ code as follows:

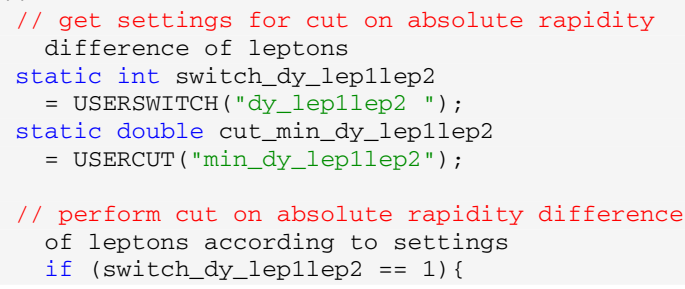

hardest leptons by defining a new xdistribution_type. The definition of the distribution in the file distribution. dat would look like

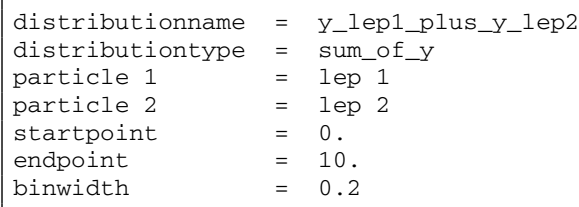


where the name and the specific definition of the binning has relevance for this example. The $\mathrm{C}++$ code for the distribution type sum_of_y can be implemented in a general way for an arbitrary number of patricle i definitions by adding an else if block to the computeobservable routine in the file xdistribution.cpp:

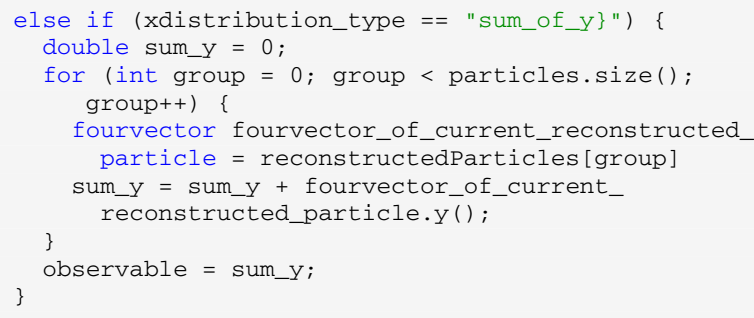

\section{Appendix C Troubleshooting}

\section{C.1 Compiling on 1xplus}

There is a problem when compiling OPENLOOPS on the lxplus cluster due to an outdated Fortran version. Furthermore, when using the window manager screen, the compilers/executables might not be working (including Python). In both cases you need to execute

$$
\begin{aligned}
& \text { \$ source /afs/cern.ch/sw/lcg/hepsoft/ } \\
& \text { 0.9/x86_64-slc6-gcc48-opt/setup.sh }
\end{aligned}
$$

before compiling OPENLOOPS. ${ }^{50}$

\section{C.2 Using a window manager on lxplus}

Since lxplus grants read/write permissions via kerberos tickets, which are valid only for $24 \mathrm{~h}$, it is not trivial to employ a window manager. In particular, the standard option screen does not work properly. We recommend tmux on lxplus instead, which can be used as follows:

First, create a session with a kerberos ticket

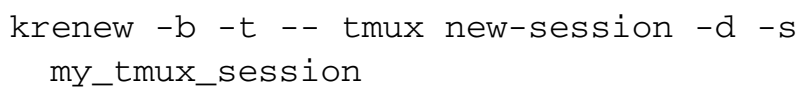

and enter the session with

tmux attach

Change the directory to a MATRIX process folder and start a run inside the tmux session. The session can now be detached ( Ctrl+b @ ) and the run will continue. However, $\mathrm{read} /$ write permission will end after $24 \mathrm{~h}$. In order to maintain

${ }^{50}$ In general, it is a good idea to add it to your .bashrc/.bash_profile (and/or your.screenrc) to avoid having to retype it for each new session/screen. them, the kerberos ticket must be renewed inside the tmux session. To do so, enter the tmux session again and open a second window inside the same session ( $\mathrm{Ctrl+b}$ ( ). Now, enter

kinit

and type your CERN password to renew the kerberos ticket. Change between the two tmux windows ( Ctrl+b n) and get back to the output of the MATRIX run. Before further $24 \mathrm{~h}$ have passed, the kerberos ticket needs to be renewed again. In principle, one could have a script take care of these renewals. However, it is not secure to safe the CERN password within a human-readable executable.

\section{C.3 Problems with libquadmath}

If you encounter

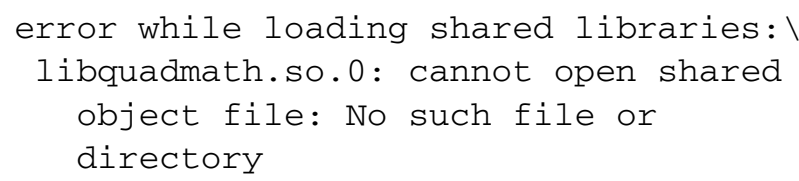

at runtime, implying that dynamic linking to $1 \mathrm{i}$ bquadmath failed, you can set path_to_libgfortran in the file MATRIX_configuration to the path where libquadmath is installed on your system.

\section{References}

1. MATRIX. http://matrix.hepforge.org/

2. R. Hamberg, W.L. van Neerven, T. Matsuura, A complete calculation of the order $\alpha_{s}^{2}$ correction to the Drell-Yan $K$ factor. Nucl. Phys. B 359, 343-405 (1991). [Erratum: Nucl. Phys. B 644, 403 (2002)]

3. R.V. Harlander, W.B. Kilgore, Next-to-next-to-leading order Higgs production at hadron colliders. Phys. Rev. Lett. 88, 201801 (2002). arXiv:hep-ph/0201206

4. C. Anastasiou, K. Melnikov, Higgs boson production at hadron colliders in NNLO QCD. Nucl. Phys. B 646, 220-256 (2002). arXiv:hep-ph/0207004

5. V. Ravindran, J. Smith, W.L. van Neerven, NNLO corrections to the total cross-section for Higgs boson production in hadron hadron collisions. Nucl. Phys. B 665, 325-366 (2003). arXiv:hep-ph/0302135

6. C. Anastasiou, L.J. Dixon, K. Melnikov, F. Petriello, High precision QCD at hadron colliders: electroweak gauge boson rapidity distributions at NNLO. Phys. Rev. D 69, 094008 (2004). arXiv:hep-ph/0312266

7. C. Anastasiou, K. Melnikov, F. Petriello, Fully differential Higgs boson production and the di-photon signal through nextto-next-to-leading order. Nucl. Phys. B 724, 197-246 (2005). arXiv:hep-ph/0501130

8. K. Melnikov, F. Petriello, Electroweak gauge boson production at hadron colliders through $\mathcal{O}\left(\alpha_{S}^{2}\right)$. Phys. Rev. D 74, 114017 (2006). arXiv:hep-ph/0609070

9. S. Catani, M. Grazzini, An NNLO subtraction formalism in hadron collisions and its application to Higgs boson pro- 
duction at the LHC. Phys. Rev. Lett. 98, 222002 (2007). arXiv:hep-ph/0703012

10. C. Anastasiou, G. Dissertori, F. Stöckli, NNLO QCD predictions for the $H \rightarrow W W \rightarrow \ell v \ell v$ signal at the LHC. JHEP 09, 018 (2007). arXiv:0707.2373

11. M. Grazzini, NNLO predictions for the Higgs boson signal in the $H \rightarrow W W \rightarrow l v l v$ and $H \rightarrow Z Z \rightarrow 4 l$ decay channels. JHEP 02, 043 (2008). arXiv:0801.3232

12. S. Catani, L. Cieri, G. Ferrera, D. de Florian, M. Grazzini, Vector boson production at hadron colliders: a fully exclusive QCD calculation at NNLO. Phys. Rev. Lett. 103, 082001 (2009). arXiv:0903.2120

13. G. Ferrera, M. Grazzini, F. Tramontano, Associated $W H$ production at hadron colliders: a fully exclusive QCD calculation at NNLO. Phys. Rev. Lett. 107, 152003 (2011). arXiv:1107.1164

14. G. Ferrera, M. Grazzini, F. Tramontano, Associated $Z H$ production at hadron colliders: the fully differential NNLO QCD calculation. Phys. Lett. B 740, 51-55 (2015). arXiv:1407.4747

15. G. Ferrera, G. Somogyi, F. Tramontano, Associated production of a Higgs boson decaying into bottom quarks at the LHC in full NNLO QCD. Phys. Lett. B 780, 346-351 (2018). arXiv: 1705.10304

16. J.M. Campbell, R.K. Ellis, C. Williams, Associated production of a Higgs boson at NNLO. JHEP 06, 179 (2016). arXiv: 1601.00658

17. R.V. Harlander, W.B. Kilgore, Higgs boson production in bottom quark fusion at next-to-next-to leading order. Phys. Rev. D 68, 013001 (2003). arXiv:hep-ph/0304035

18. R.V. Harlander, K.J. Ozeren, M. Wiesemann, Higgs plus jet production in bottom quark annihilation at next-to-leading order. Phys. Lett. B 693, 269-273 (2010). arXiv:1007.5411

19. R. Harlander, M. Wiesemann, Jet-veto in bottom-quark induced Higgs production at next-to-next-to-leading order. JHEP 04, 066 (2012). arXiv:1111.2182

20. S. Bühler, F. Herzog, A. Lazopoulos, R. Müller, The fully differential hadronic production of a Higgs boson via bottom quark fusion at NNLO. JHEP 07, 115 (2012). arXiv:1204.4415

21. S. Marzani, R.D. Ball, V. Del Duca, S. Forte, A. Vicini, Higgs production via gluon-gluon fusion with finite top mass beyond next-to-leading order. Nucl. Phys. B 800, 127-145 (2008). arXiv:0801.2544

22. R.V. Harlander, K.J. Ozeren, Finite top mass effects for hadronic Higgs production at next-to-next-to-leading order. JHEP 11, 088 (2009). arXiv:0909.3420

23. R.V. Harlander, H. Mantler, S. Marzani, K.J. Ozeren, Higgs production in gluon fusion at next-to-next-to-leading order QCD for finite top mass. Eur. Phys. J. C 66, 359-372 (2010). arXiv:0912.2104

24. A. Pak, M. Rogal, M. Steinhauser, Finite top quark mass effects in NNLO Higgs boson production at LHC. JHEP 02, 025 (2010). arXiv:0911.4662

25. T. Neumann, M. Wiesemann, Finite top-mass effects in gluoninduced Higgs production with a jet-veto at NNLO. JHEP 11, 150 (2014). arXiv: 1408.6836

26. D. de Florian, J. Mazzitelli, Higgs Boson pair production at nextto-next-to-leading order in QCD. Phys. Rev. Lett. 111, 201801 (2013). arXiv:1309.6594

27. D. de Florian, M. Grazzini, C. Hanga, S. Kallweit, J.M. Lindert, P. Maierhöfer, J. Mazzitelli, D. Rathlev, Differential higgs boson pair production at next-to-next-to-leading order in QCD. JHEP 09, 151 (2016). arXiv:1606.09519

28. S. Catani, L. Cieri, D. de Florian, G. Ferrera, M. Grazzini, Diphoton production at hadron colliders: a fully-differential QCD calculation at NNLO. Phys. Rev. Lett. 108, 072001 (2012). arXiv:1110.2375. [Erratum: Phys. Rev. Lett. 117(8), 089901 (2016)]
29. J.M. Campbell, R.K. Ellis, Y. Li, C. Williams, Predictions for diphoton production at the LHC through NNLO in QCD. JHEP 07, 148 (2016). arXiv: 1603.02663

30. M. Grazzini, S. Kallweit, D. Rathlev, A. Torre, $Z \gamma$ production at hadron colliders in NNLO QCD. Phys. Lett. B 731, 204-207 (2014). arXiv:1309.7000

31. M. Grazzini, S. Kallweit, D. Rathlev, $W \gamma$ and $Z \gamma$ production at the LHC in NNLO QCD. JHEP 07, 085 (2015). arXiv: 1504.01330

32. J.M. Campbell, T. Neumann, C. Williams, $Z \gamma$ production at NNLO including anomalous couplings. JHEP. 11, 150 (2017). arXiv: 1708.02925

33. F. Cascioli, T. Gehrmann, M. Grazzini, S. Kallweit, P. Maierhöfer, A. von Manteuffel, S. Pozzorini, D. Rathlev, L. Tancredi, E. Weihs, ZZ production at hadron colliders in NNLO QCD. Phys. Lett. B 735, 311-313 (2014). arXiv:1405.2219

34. M. Grazzini, S. Kallweit, D. Rathlev, ZZ production at the LHC: fiducial cross sections and distributions in NNLO QCD. Phys. Lett. B 750, 407-410 (2015). arXiv: 1507.06257

35. G. Heinrich, S. Jahn, S.P. Jones, M. Kerner, J. Pires, NNLO predictions for Z-boson pair production at the LHC. JHEP. 03, 142 (2018). arXiv:1710.06294

36. T. Gehrmann, M. Grazzini, S. Kallweit, P. Maierhöfer, A. von Manteuffel, S. Pozzorini, D. Rathlev, L. Tancredi, $W^{+} W^{-}$production at hadron colliders in next to next to leading order QCD. Phys. Rev. Lett. 113(21), 212001 (2014). arXiv:1408.5243

37. M. Grazzini, S. Kallweit, S. Pozzorini, D. Rathlev, M. Wiesemann, $W^{+} W$ production at the LHC: fiducial cross sections and distributions in NNLO QCD. JHEP 08, 140 (2016). arXiv: 1605.02716

38. M. Grazzini, S. Kallweit, D. Rathlev, M. Wiesemann, $W^{ \pm} Z$ production at hadron colliders in NNLO QCD. Phys. Lett. B 761, 179-183 (2016). arXiv:1604.08576

39. M. Grazzini, S. Kallweit, D. Rathlev, M. Wiesemann, $W^{ \pm} Z$ production at the LHC: fiducial cross sections and distributions in NNLO QCD. JHEP 05, 139 (2017). arXiv: 1703.09065

40. M. Czakon, P. Fiedler, A. Mitov, Total top-quark pair-production cross section at hadron colliders through $\mathcal{O}\left(\alpha_{S}^{4}\right)$. Phys. Rev. Lett. 110, 252004 (2013). arXiv:1303.6254

41. M. Czakon, D. Heymes, A. Mitov, High-precision differential predictions for top-quark pairs at the LHC. Phys. Rev. Lett. 116(8), 082003 (2016). arXiv: 1511.00549

42. M. Brucherseifer, F. Caola, K. Melnikov, On the NNLO QCD corrections to single-top production at the LHC. Phys. Lett. B 736, 58-63 (2014). arXiv:1404.7116

43. E.L. Berger, J. Gao, C.P. Yuan, H.X. Zhu, NNLO QCD corrections to t-channel single top-quark production and decay. Phys. Rev. D 94(7), 071501 (2016). arXiv:1606.08463

44. J. Currie, A. Gehrmann-De Ridder, T. Gehrmann, E.W.N. Glover, A. Huss, J. Pires, Precise predictions for dijet production at the LHC. Phys. Rev. Lett. 119(15), 152001 (2017). arXiv:1705.10271

45. M. Cacciari, F.A. Dreyer, A. Karlberg, G.P. Salam, G. Zanderighi, Fully differential vector-boson-fusion Higgs production at nextto-next-to-leading order. Phys. Rev. Lett. 115(8), 082002 (2015). arXiv: 1506.02660

46. R. Boughezal, C. Focke, W. Giele, X. Liu, F. Petriello, Higgs boson production in association with a jet at NNLO using jettiness subtraction. Phys. Lett. B 748, 5-8 (2015). arXiv: 1505.03893

47. F. Caola, K. Melnikov, M. Schulze, Fiducial cross sections for Higgs boson production in association with a jet at next-to-nextto-leading order in QCD. Phys. Rev. D 92(7), 074032 (2015). arXiv: 1508.02684

48. X. Chen, J. Cruz-Martinez, T. Gehrmann, E.W.N. Glover, M. Jaquier, NNLO QCD corrections to Higgs boson production at large transverse momentum. JHEP 10, 066 (2016). arXiv: 1607.08817 
49. J.M. Campbell, R.K. Ellis, C. Williams, Direct photon production at next-to-next-to-leading order. Phys. Rev. Lett. 118(22), 222001 (2017). arXiv: 1612.04333

50. R. Boughezal, J.M. Campbell, R.K. Ellis, C. Focke, W.T. Giele, $\mathrm{X}$. Liu, F. Petriello, Z-boson production in association with a jet at next-to-next-to-leading order in perturbative QCD. Phys. Rev. Lett. 116(15), 152001 (2016). arXiv: 1512.01291

51. A. Gehrmann-De Ridder, T. Gehrmann, E.W.N. Glover, A. Huss, T.A. Morgan, NNLO QCD corrections for Drell-Yan $p_{T}^{Z}$ and $\phi^{*}$ observables at the LHC. JHEP 11, 094 (2016). arXiv:1610.01843

52. R. Boughezal, C. Focke, X. Liu, F. Petriello, $W$-boson production in association with a jet at next-to-next-to-leading order in perturbative QCD. Phys. Rev. Lett. 115(6), 062002 (2015). arXiv: 1504.02131

53. R. Gavin, Y. Li, F. Petriello, S. Quackenbush, W physics at the LHC with FEWZ 2.1. Comput. Phys. Commun. 184, 208-214 (2013). arXiv:1201.5896

54. C. Anastasiou, S. Bucherer, Z. Kunszt, HPro: a NLO Monte-Carlo for Higgs production via gluon fusion with finite heavy quark masses. JHEP 10, 068 (2009). arXiv:0907.2362

55. R. Boughezal, J.M. Campbell, R.K. Ellis, C. Focke, W. Giele, X. Liu, F. Petriello, C. Williams, Color singlet production at NNLO in MCFM. Eur. Phys. J. C 77(1), 7 (2017). arXiv:1605.08011

56. S. Catani, M. Seymour, The dipole formalism for the calculation of QCD jet cross-sections at next-to-leading order. Phys. Lett. B 378, 287-301 (1996). arXiv:hep-ph/9602277

57. S. Catani, M. Seymour, A general algorithm for calculating jet cross-sections in NLO QCD. Nucl. Phys. B 485, 291-419 (1997). arXiv:hep-ph/9605323

58. F. Cascioli, P. Maierhöfer, S. Pozzorini, Scattering amplitudes with open loops. Phys. Rev. Lett. 108, 111601 (2012). arXiv: 1111.5206

59. F. Buccioni, S. Pozzorini, M. Zoller, On-the-fly reduction of open loops. Eur. Phys. J. C 78(1), 70 (2018). arXiv:1710.11452

60. T. Gehrmann, L. Tancredi, Two-loop QCD helicity amplitudes for $q \bar{q} \rightarrow W^{ \pm} \gamma$ and $q \bar{q} \rightarrow Z^{0} \gamma$. JHEP 02, 004 (2012). arXiv: 1112.1531

61. T. Gehrmann, A. von Manteuffel, L. Tancredi, The two-loop helicity amplitudes for $q \bar{q}^{\prime} \rightarrow V_{1} V_{2} \rightarrow$ 4leptons. arXiv:1503.04812

62. D. de Florian, J. Mazzitelli, Two-loop virtual corrections to Higgs pair production. Phys. Lett. B 724, 306-309 (2013). arXiv: 1305.5206

63. M. Grazzini, S. Kallweit, D. Rathlev, M. Wiesemann, Transversemomentum resummation for vector-boson pair production at NNLL + NNLO. JHEP 08, 154 (2015). arXiv:1507.02565

64. D.A. Kosower, Antenna factorization of gauge theory amplitudes. Phys. Rev. D 57, 5410-5416 (1998). arXiv:hep-ph/9710213

65. A. Gehrmann-De Ridder, T. Gehrmann, E.W.N. Glover, Antenna subtraction at NNLO. JHEP 09, 056 (2005). arXiv:hep-ph/0505111

66. A. Daleo, T. Gehrmann, D. Maitre, Antenna subtraction with hadronic initial states. JHEP 04, 016 (2007). arXiv:hep-ph/0612257

67. J. Currie, E.W.N. Glover, S. Wells, Infrared structure at NNLO using antenna subtraction. JHEP 04, 066 (2013). arXiv: 1301.4693

68. G. Somogyi, Z. Trocsanyi, V. Del Duca, Matching of singly- and doubly-unresolved limits of tree-level QCD squared matrix elements. JHEP 06, 024 (2005). arXiv:hep-ph/0502226

69. V. Del Duca, C. Duhr, G. Somogyi, F. Tramontano, Z. Trocsanyi, Higgs boson decay into b-quarks at NNLO accuracy. JHEP 04, 036 (2015). arXiv: 1501.07226

70. V. Del Duca, C. Duhr, A. Kardos, G. Somogyi, Z. Trocsanyi, Three-jet production in electron-positron collisions at next-tonext-to-leading order accuracy. Phys. Rev. Lett. 117(15), 152004 (2016). arXiv: 1603.08927
71. M. Czakon, A novel subtraction scheme for double-real radiation at NNLO. Phys. Lett. B 693, 259-268 (2010). arXiv:1005.0274

72. M. Czakon, Double-real radiation in hadronic top quark pair production as a proof of a certain concept. Nucl. Phys. B 849, 250-295 (2011). arXiv:1101.0642

73. M. Czakon, D. Heymes, Four-dimensional formulation of the sector-improved residue subtraction scheme. Nucl. Phys. B 890, 152-227 (2014). arXiv:1408.2500

74. R. Boughezal, X. Liu, F. Petriello, $N$-jettiness soft function at next-to-next-to-leading order. Phys. Rev. D 91(9), 094035 (2015). arXiv: 1504.02540

75. J. Gaunt, M. Stahlhofen, F.J. Tackmann, J.R. Walsh, $N$-jettiness subtractions for NNLO QCD calculations. JHEP 09, 058 (2015). arXiv: 1505.04794

76. F. Caola, K. Melnikov, R. Röntsch, Nested soft-collinear subtractions in NNLO QCD computations. Eur. Phys. J. C 77(4), 248 (2017). arXiv: 1702.01352

77. J.C. Collins, D.E. Soper, G.F. Sterman, Transverse momentum distribution in Drell-Yan pair and $W$ and $Z$ boson production. Nucl. Phys. B 250, 199 (1985)

78. G. Bozzi, S. Catani, D. de Florian, M. Grazzini, Transversemomentum resummation and the spectrum of the Higgs boson at the LHC. Nucl. Phys. B 737, 73-120 (2006). arXiv:hep-ph/0508068

79. R. Bonciani, S. Catani, M. Grazzini, H. Sargsyan, A. Torre, The $q_{T}$ subtraction method for top quark production at hadron colliders. Eur. Phys. J. C 75(12), 581 (2015). arXiv:1508.03585

80. S. Frixione, Z. Kunszt, A. Signer, Three jet cross-sections to next-to-leading order. Nucl. Phys. B 467, 399-442 (1996). arXiv:hep-ph/9512328

81. S. Frixione, A General approach to jet cross-sections in QCD. Nucl. Phys. B 507, 295-314 (1997). arXiv:hep-ph/9706545

82. D. de Florian, M. Grazzini, The structure of large logarithmic corrections at small transverse momentum in hadronic collisions. Nucl. Phys. B 616, 247-285 (2001). arXiv:hep-ph/0108273

83. S. Catani, L. Cieri, D. de Florian, G. Ferrera, M. Grazzini, Universality of transverse-momentum resummation and hard factors at the NNLO. Nucl. Phys. B 881, 414-443 (2014). arXiv:1311.1654

84. S. Catani, M. Grazzini, Higgs boson production at hadron colliders: hard-collinear coefficients at the NNLO. Eur. Phys. J. C 72, 2013 (2012). arXiv: 1106.4652

85. S. Catani, L. Cieri, D. de Florian, G. Ferrera, M. Grazzini, Vector boson production at hadron colliders: hard-collinear coefficients at the NNLO. Eur. Phys. J. C 72, 2195 (2012). arXiv: 1209.0158

86. S. Kallweit, J.M. Lindert, P. Maierhöfer, S. Pozzorini, M. Schönherr, NLO electroweak automation and precise predictions for $W+$ multijet production at the LHC. JHEP 04, 012 (2015). arXiv: 1412.5157

87. S. Kallweit, J.M. Lindert, P. Maierhöfer, S. Pozzorini, M. Schönherr, NLO QCD + EW predictions for $V+$ jets including off-shell vector-boson decays and multijet merging. JHEP 04, 021 (2016). arXiv: 1511.08692

88. S. Catani, S. Dittmaier, M.H. Seymour, Z. Trocsanyi, The dipole formalism for next-to-leading order QCD calculations with massive partons. Nucl. Phys. B 627, 189-265 (2002). arXiv:hep-ph/0201036

89. A. Denner, S. Dittmaier, L. Hofer, COLLIER—a fortran-library for one-loop integrals. PoS LL2014, 071 (2014). arXiv:1407.0087

90. A. Denner, S. Dittmaier, L. Hofer, Collier: a fortran-based complex one-loop library in extended regularizations. Comput. Phys. Commun. 212, 220-238 (2017). arXiv: 1604.06792

91. G. Ossola, C.G. Papadopoulos, R. Pittau, CutTools: a program implementing the OPP reduction method to compute one-loop amplitudes. JHEP 0803, 042 (2008). arXiv:0711.3596 
92. A. van Hameren, OneLOop: for the evaluation of one-loop scalar functions. Comput. Phys. Commun. 182, 2427-2438 (2011). arXiv: 1007.4716

93. M. Wiesemann, Transverse-momentum resummation of colorless final states at the NNLL + NNLO. PoS RADCOR2015, 026 (2016). arXiv: 1602.03401

94. C. Anastasiou, E.W.N. Glover, M.E. Tejeda-Yeomans, Two loop QED and QCD corrections to massless fermion boson scattering. Nucl. Phys. B 629, 255-289 (2002). arXiv:hep-ph/0201274

95. The VVAMP project, by T. Gehrmann, A. von Manteuffel, and L. Tancredi, is publicly available at http://vvamp.hepforge.org

96. A. Buckley, J. Ferrando, S. Lloyd, K. Nordström, B. Page, M. Rüfenacht, M. Schönherr, G. Watt, LHAPDF6: parton density access in the LHC precision era. Eur. Phys. J. C 75, 132 (2015). arXiv: 1412.7420

97. The OPEnLoops one-loop generator, by F. Cascioli, J. Lindert, P. Maierhöfer, and S. Pozzorini, is publicly available at http:// openloops.hepforge.org

98. C.W. Bauer, A. Frink, R. Kreckel, Introduction to the GiNaC framework for symbolic computation within the $\mathrm{C}++$ programming language. J. Symb. Comput. 33, 1 (2000). arXiv:cs/0004015

99. Y.L. Dokshitzer, G.D. Leder, S. Moretti, B.R. Webber, Better jet clustering algorithms. JHEP 08, 001 (1997). arXiv:hep-ph/9707323

100. M. Wobisch, T. Wengler, Hadronization corrections to jet crosssections in deep inelastic scattering, in Monte Carlo generators for HERA physics. In: Proceedings, Workshop, Hamburg, Germany, vol. 1998-1999, pp. 270-279 (1998). arXiv:hep-ph/9907280

101. S. Catani, Y.L. Dokshitzer, M.H. Seymour, B.R. Webber, Longitudinally invariant $K_{t}$ clustering algorithms for hadron hadron collisions. Nucl. Phys. B 406, 187-224 (1993)

102. M. Cacciari, G.P. Salam, G. Soyez, The Anti- $k(t)$ jet clustering algorithm. JHEP 0804, 063 (2008). arXiv:0802.1189

103. S. Frixione, Isolated photons in perturbative QCD. Phys. Lett. B 429, 369-374 (1998). arXiv:hep-ph/9801442

104. P.Z. Skands et al., SUSY Les Houches accord: interfacing SUSY spectrum calculators, decay packages, and event generators. JHEP 07, 036 (2004). arXiv:hep-ph/0311123

105. Particle Data Group Collaboration, C. Patrignani et al., Review of particle physics. Chin. Phys. C 40(10), 100001 (2016)

106. ATLAS Collaboration, G. Aad et al., Measurements of $W \gamma$ and $Z \gamma$ production in $p p$ collisions at $\sqrt{s}=7 \mathrm{TeV}$ with the ATLAS detector at the LHC. Phys. Rev. D 87(11), 112003 (2013). arXiv:1302.1283 [Erratum: Phys. Rev. D 91(11), 119901 (2015)]

107. ATLAS Collaboration, G. Aad et al., Measurements of $W^{ \pm} Z$ production cross sections in $p p$ collisions at $\sqrt{s}=8 \mathrm{TeV}$ with the ATLAS detector and limits on anomalous gauge boson selfcouplings. Phys. Rev. D 93(9), 092004 (2016). arXiv:1603.02151

108. A. Denner, S. Dittmaier, M. Roth, L.H. Wieders, Electroweak corrections to charged-current $e^{+} e^{-} \rightarrow 4$ fermion processes: Technical details and further results. Nucl. Phys. B 724, 247-294 (2005). arXiv:hep-ph/0505042. [Erratum: Nucl. Phys. B 854, 504 (2012)]

109. NNPDF Collaboration, R.D. Ball et al., Parton distributions for the LHC Run II. JHEP. 1504, 040, (2015). arXiv:1410.8849

110. R.V. Harlander, S. Liebler, H. Mantler, SusHi: a program for the calculation of Higgs production in gluon fusion and bottom-quark annihilation in the standard model and the MSSM. Comput. Phys. Commun. 184, 1605-1617 (2013). arXiv:1212.3249

111. S. Frixione, G. Ridolfi, Jet photoproduction at HERA. Nucl. Phys. B 507, 315-333 (1997). arXiv:hep-ph/9707345

112. S. Alioli et al., Precision studies of observables in $p p \rightarrow W \rightarrow l v_{l}$ and $p p \rightarrow \gamma, Z \rightarrow l^{+} l^{-}$processes at the LHC. Eur. Phys. J. C 77(5), 280 (2017). arXiv:1606.02330

113. ATLAS Collaboration, M. Aaboud et al., Precision measurement and interpretation of inclusive $W^{+}, W^{-}$and $Z / \gamma^{*}$ production cross sections with the ATLAS detector. Eur. Phys. J. C 77(6), 367 (2017). arXiv: 1612.03016

114. K.O. Mikaelian, M.A. Samuel, D. Sahdev, The magnetic moment of weak bosons produced in $p p$ and $p$ anti- $p$ collisions. Phys. Rev. Lett. 43, 746 (1979)

115. F. Caola, K. Melnikov, R. Röntsch, L. Tancredi, QCD corrections to ZZ production in gluon fusion at the LHC. Phys. Rev. D 92(9), 094028 (2015). arXiv:1509.06734

116. F. Caola, K. Melnikov, R. Röntsch, L. Tancredi, QCD corrections to $W^{+} W^{-}$production through gluon fusion. Phys. Lett. B 754, 275-280 (2016). arXiv: 1511.08617

117. U. Baur, T. Han, J. Ohnemus, Amplitude zeros in $W^{ \pm} Z$ production. Phys. Rev. Lett. 72, 3941-3944 (1994). arXiv:hep-ph/9403248

118. S. Catani, B.R. Webber, Infrared safe but infinite: soft gluon divergences inside the physical region. JHEP 10, 005 (1997). arXiv:hep-ph/9710333

119. A. Gehrmann-De Ridder, T. Gehrmann, E.W.N. Glover, A. Huss, T.A. Morgan, The NNLO QCD corrections to $Z$ boson production at large transverse momentum. JHEP 07, 133 (2016). arXiv: 1605.04295

120. ATLAS Collaboration, G. Aad et al., Measurements of $Z \gamma$ and $Z \gamma \gamma$ production in $p p$ collisions at $\sqrt{s}=8 \mathrm{TeV}$ with the ATLAS detector. Phys. Rev. D 93(11), 112002 (2016). arXiv:1604.05232

121. CMS Collaboration, Measurement of the production cross section for $p p \rightarrow Z \gamma \rightarrow v \bar{v} \gamma$ at $\sqrt{s}=13 \mathrm{TeV}$ at CMS, CMS-PAS-SMP16-004

122. C.M.S. Collaboration, A.M. Sirunyan et al., Measurements of the $\mathrm{pp} \rightarrow W \gamma \gamma$ and $\mathrm{pp} \rightarrow Z \gamma \gamma$ cross sections and limits on anomalous quartic gauge couplings at $\sqrt{s}=8 \mathrm{TeV}$. JHEP 10, 072 (2017). arXiv: 1704.00366

123. ATLAS Collaboration, G. Aad et al., Measurements of four-lepton production in $p p$ collisions at $\sqrt{s}=8 \mathrm{TeV}$ with the ATLAS detector. Phys. Lett. B 753, 552-572 (2016). arXiv:1509.07844

124. ATLAS Collaboration, M. Aaboud et al., Measurement of the $Z Z$ production cross section in proton-proton collisions at $\sqrt{s}=$ $8 \mathrm{TeV}$ using the $Z Z \rightarrow \ell^{-} \ell^{+} \ell^{\prime-} \ell^{\prime+}$ and $Z Z \rightarrow \ell^{-} \ell^{+} \nu \bar{v}$ channels with the ATLAS detector. JHEP 01, 099 (2017). arXiv: 1610.07585

125. ATLAS Collaboration, M. Aaboud et al., $Z Z \rightarrow \ell^{+} \ell^{-} \ell^{\prime+} \ell^{\prime-}$ cross-section measurements and search for anomalous triple gauge couplings in $13 \mathrm{TeV} p p$ collisions with the ATLAS detector. Phys. Rev. D 97(3), 032005 (2018). arXiv:1709.07703

126. CMS Collaboration, Measurement of the differential cross section for $p p \rightarrow Z Z \rightarrow 4 \ell$ produced in association with jets in $p p$ collisions at $\sqrt{s}=8 \mathrm{TeV}$. CMS-PAS-SMP-15-012

127. CMS Collaboration, Measurement of the $\mathrm{ZZ}$ production cross section in $\ell \ell \ell^{\prime} \ell^{\prime}$ decays in pp collisions at $\sqrt{s}=13 \mathrm{TeV}$. CMSPAS-SMP-15-005

128. CMS Collaboration, V. Khachatryan et al., Measurement of the $\mathrm{ZZ}$ production cross section and $\mathrm{Z} \rightarrow \ell^{+} \ell^{-} \ell^{\prime+} \ell^{\prime-}$ branching fraction in pp collisions at $\sqrt{s}=13 \mathrm{TeV}$. Phys. Lett. B 763, 280-303 (2016). arXiv:1607.08834 [Erratum: Phys. Lett. B 772, 884(2017)]

129. CMS Collaboration, A.M. Sirunyan et al., Measurements of the $\mathrm{pp} \rightarrow Z Z$ production cross section and the $Z \rightarrow 4 \ell$ branching fraction, and constraints on anomalous triple gauge couplings at $\sqrt{s}=13 \mathrm{TeV}$. Eur. Phys. J. C 78(3), 165 (2018). arXiv: 1709.08601

130. ATLAS Collaboration, G. Aad et al., Measurement of total and differential $W^{+} W^{-}$production cross sections in proton-proton collisions at $\sqrt{s}=8 \mathrm{TeV}$ with the ATLAS detector and limits on anomalous triple-gauge-boson couplings. JHEP 09, 029 (2016). arXiv: 1603.01702

131. ATLAS Collaboration, M. Aaboud et al., Measurement of $W^{+} W^{-}$ production in association with one jet in proton-proton collisions 
at $\sqrt{s}=8 \mathrm{TeV}$ with the ATLAS detector. Phys. Lett. B 763, 114-133 (2016). arXiv: 1608.03086

132. ATLAS Collaboration, M. Aaboud et al., Measurement of the $W^{+} W^{-}$production cross section in $p p$ collisions at a centre-ofmass energy of $\sqrt{s}=13 \mathrm{TeV}$ with the ATLAS experiment. Phys. Lett. B 773, 354-374 (2017). arXiv:1702.04519

133. CMS Collaboration, V. Khachatryan et al., Measurement of the $\mathrm{W}^{+} \mathrm{W}^{-}$cross section in pp collisions at $\sqrt{s}=8 \mathrm{TeV}$ and limits on anomalous gauge couplings. Eur. Phys. J. C 76(7), 401 (2016). arXiv: 1507.03268

134. CMS Collaboration, Measurement of the WW cross section pp collisions at $\sqrt{s}=13 T e V$. CMS-PAS-SMP-16-006

135. ATLAS Collaboration, M. Aaboud et al., Measurement of the $W^{ \pm} Z$ boson pair-production cross section in $p p$ collisions at $\sqrt{s}=13 \mathrm{TeV}$ with the ATLAS Detector. Phys. Lett. B 762, 1-22 (2016). arXiv: 1606.04017

136. ATLAS Collaboration, Measurement of $W^{ \pm} Z$ boson pairproduction in $p p$ collisions at $\sqrt{s}=13 \mathrm{TeV}$ with the ATLAS Detector and confidence intervals for anomalous triple gauge boson couplings. ATLAS-CONF-2016-043
137. CMS Collaboration, V. Khachatryan et al., Measurement of the WZ production cross section in pp collisions at $\sqrt{s}=7$ and $8 \mathrm{TeV}$ and search for anomalous triple gauge couplings at $\sqrt{s}=8 \mathrm{TeV}$, Eur. Phys. J. C 77(4), 236 (2017). arXiv:1609.05721

138. CMS Collaboration, V. Khachatryan et al., Measurement of the WZ production cross section in pp collisions at $\sqrt{(} s)=13 \mathrm{TeV}$, Phys. Lett. B 766, 268-290 (2017). arXiv:1607.06943

139. LHC Higgs Cross Section Working Group Collaboration, D. de Florian et al., Handbook of LHC Higgs cross sections: 4. Deciphering the nature of the Higgs sector. (2016). arXiv:1610.07922

140. CMS Collaboration, S. Chatrchyan et al., Measurement of the $W \gamma$ and $Z \gamma$ inclusive cross sections in $p p$ collisions at $\sqrt{s}=7 \mathrm{TeV}$ and limits on anomalous triple gauge boson couplings. Phys. Rev. D 89(9), 092005 (2014). arXiv:1308.6832 